
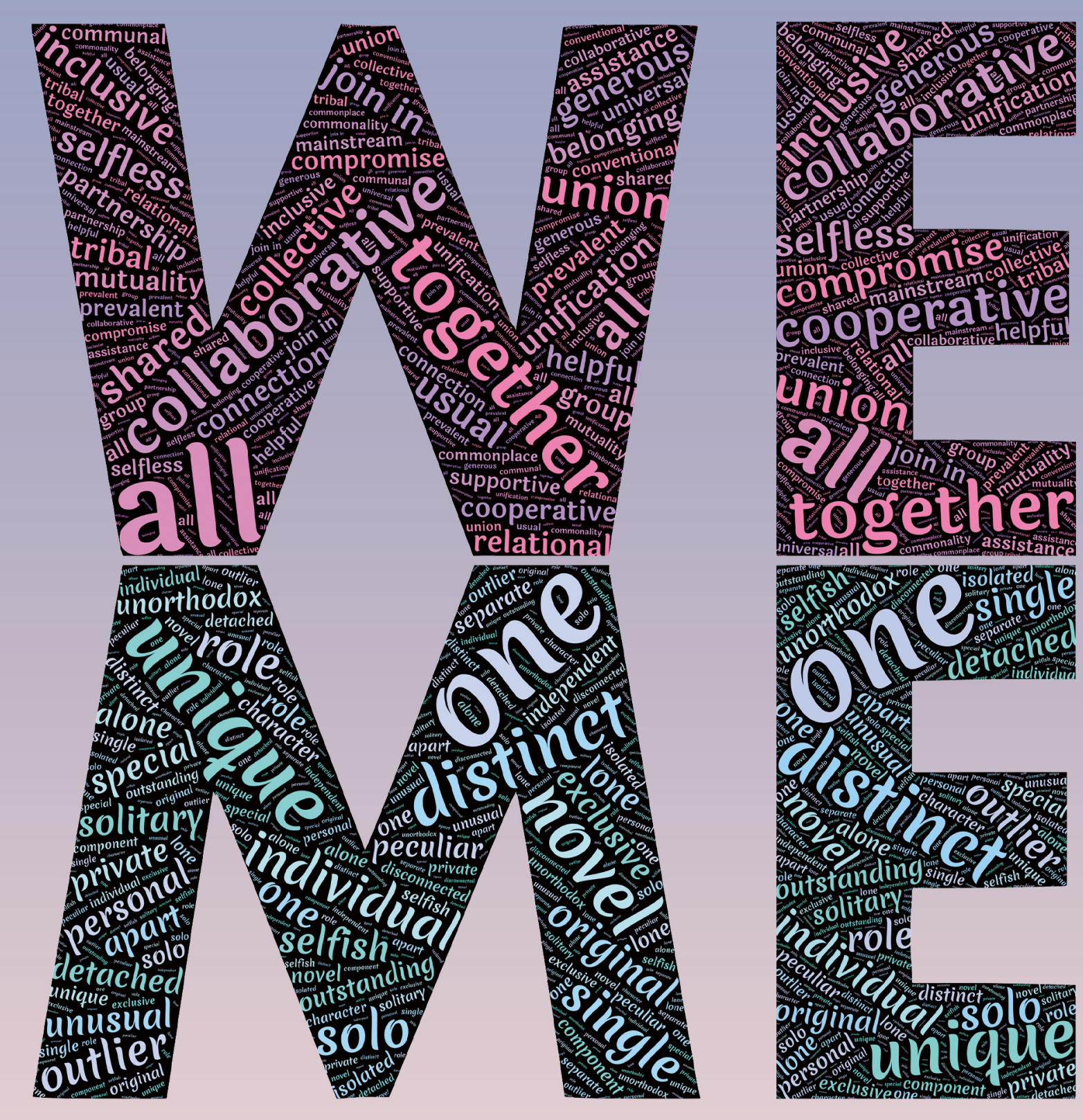

है

COMPLAINT, COMPROMISE AND SOLUTION CONCEPTS

FOR COOPERATIVE GAMES

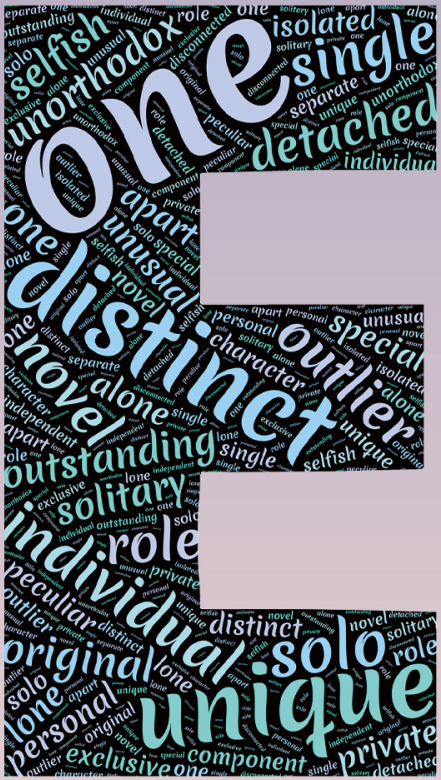

wellcograte

(1) (1)

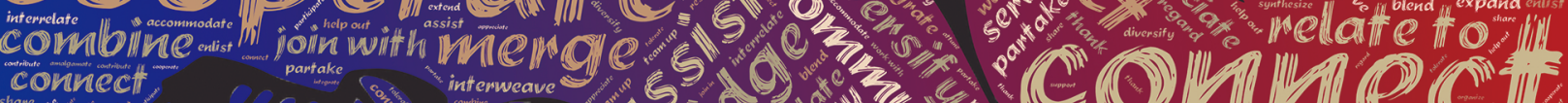
comnect

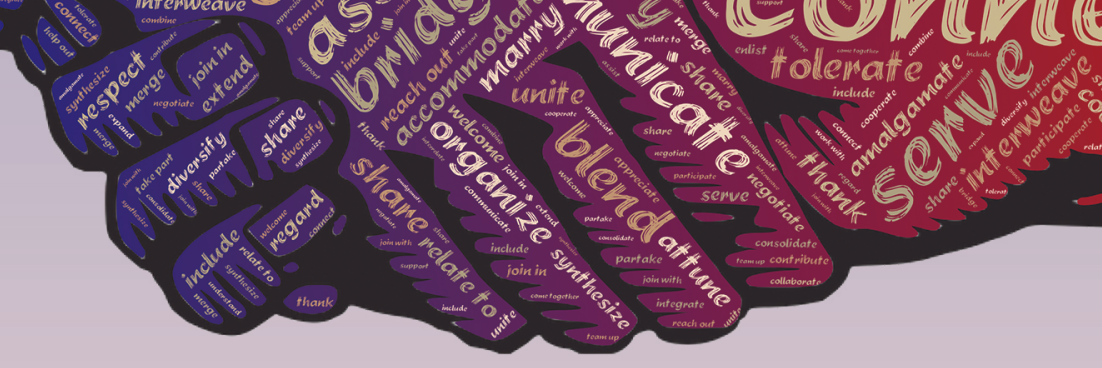

\title{
Panfei Sun
}




\section{COMPLAINT, COMPROMISE AND SOLUTION CONCEPTS FOR COOPERATIVE GAMES}

Panfei Sun 



\section{COMPLAINT, COMPROMISE AND SOLUTION CONCEPTS FOR COOPERATIVE GAMES}

\section{DISSERTATION}

to obtain

the degree of doctor at the University of Twente, on the authority of the rector magnificus, prof.dr. T.T.M. Palstra, on account of the decision of the graduation committee, to be publicly defended on Wednesday the 20th of February 2019 at 16:45 hrs

by

\section{Panfei Sun}

born on the 21st of April 1993

in Shenqiu, China 
This dissertation has been approved by the supervisors prof.dr. M. Uetz, prof. dr. H. Sun and the co-supervisor dr. W. Kern

The research reported in this thesis has been carried out within the framework of the MEMORANDUM OF AGREEMENT FOR A DOUBLE DOCTORATE DEGREE BETWEEN NORTHWESTERN POLYTECHNICAL UNIVERSITY, PEOPLE'S REPUBLIC OF CHINA AND THE UNIVERSITY OF TWENTE, THE NETHERLANDS

DSI Ph.D. Thesis Series No.19-004

UNIVERSITY | DIGITAL SOCIETY Digital Society Institute

OF TWENTE. INSTITUTE P.O. Box 217, 7500 AE Enschede, The Netherlands.

ISBN: 978-90-365-4719-2

ISSN: 2589-7721 (DSI Ph.D. thesis Series No.19-004)

DOI: $10.3990 / 1.9789036547192$

Available online at https://doi.org/10.3990/1.9789036547192

Typeset with $\mathrm{BT}_{\mathrm{E}} \mathrm{X}$

Printed by Ipskamp Printing, Enschede

Cover design by Panfei Sun

Copyright (c)2019 Panfei Sun, Enschede, The Netherlands

All rights reserved. No part of this work may be reproduced, stored in a retrieval system, or transmitted in any form or by any means, electronic, mechanical, photocopying, recording, or otherwise, without prior permission from the copyright owner. 


\section{Graduation Committee}

Chairman/secretary:

prof. dr. J.N. Kok

University of Twente

Supervisors:

prof. dr. M.J. Uetz

University of Twente

prof. dr. H. Sun

Northwestern Polytechnical University

Co-Supervisor:

dr. W. Kern

University of Twente

Referee:

dr. R.A.M.G. Joosten

University of Twente

Members:

prof. dr. F. Thuijsman

Maastricht University

prof. dr. J.L. Hurink

University of Twente

prof. dr. G. Xu

Northwestern Polytechnical University

dr. B. Manthey

University of Twente

dr. D. Hou

Northwestern Polytechnical University 



\section{Preface}

This thesis focuses on the area of solution concepts for cooperative games with transferable utility. The content consists of research results by the author when he studied as a PhD student both at Northwestern Polytechnical University and University of Twente. Apart from the introductory chapter, the other chapters in this thesis are based on corresponding research papers, which are in different stages of the publication process, some have been published or accepted already, some have been submitted, others have been completed, written by the author in the last four years.

In the introductory chapter, we introduce the fundamental models and examples of cooperative theory, including different classical solution concepts. Most of the necessary terminology and notations that will be used in the subsequent chapters are listed In this chapter. Terms and notations used only in some specific situations are listed in the corresponding chapters.

Chapter 2 and 3 investigate the ( $\alpha$-)ENSC value in both procedural and optimization method, and we also provide characterizations for these two values. As an application of the method presented in the former two chapters, Chapter 4 deals with the allocation problem in sharing the cost of cleaning a polluted river. A new sharing method is defined and characterizations are provided based on different principles. In Chapter 5, we propose a more general compromise solution concept and also reveal the relations with some other well known compromise values. In Chapter 6 , we continue to study the compromise value but in an alternative way, which was motivated by the definition of the nucleolus [71]. Furthermore, in Chapter 7, we derive values for cooperative games with stochastic payoffs as solutions to various 
minimization problems.

\section{Papers underlying this thesis}

[1] Optimization implementation and characterization of the equal allocation of non-separable costs value, J Optim Theory 173 (2017), 336-352 (with D. Hou, H. Sun and T. Driessen).

(Chapter 2)

[2] Process and optimization implementation of the $\alpha$-ENSC value, Math Meth Oper Res, 86 (2018), 293-308. (with D. Hou, H. Sun and H. Zhang). (Chapter 3)

[3] Responsibility and sharing the cost of cleaning a polluted river, revision being processed (with D. Hou and H. Sun).

(Chapter 4)

[4] The general compromise value for cooperative games with transferable utility, submitted (with D. Hou and H. Sun).

(Chapter 5)

[5] Compromise for the complaint: an optimization approach to the ENSC value and the CIS value, J. Oper. Res. Soc. 69-4 (2017), 571-579 (with D. Hou, G. $\mathrm{Xu}$ and T. Driessen) .

(Chapter 6)

[6] Compromise for the per capita complaint: an optimization characterization of two equalitarian values, submitted (with D. Hou, A. Lardon and T. Driessen) .

(Chapter 6)

[7] Optimal solution concepts for cooperative games with stochastic payoffs, submitted (with D. Hou, H. Sun and W. Kern).

(Chapter 7) 


\section{Contents}

Preface vii

1 Introduction 1

1.1 Game Theory.......................... 1

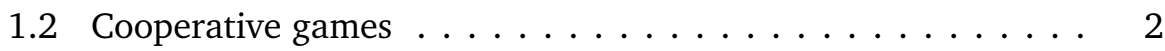

1.3 Solution concepts for cooperative games . . . . . . . . . 5

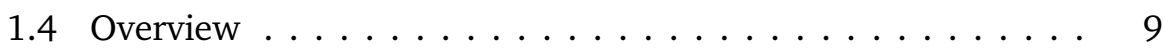

2 Implementation of the ENSC value $\quad 13$

2.1 Introduction . . . . . . . . . . . . . 13

2.2 Optimization implementation of the ENSC value . . . . . . . 15

2.2.1 Optimization model based on the least square criterion 15

2.2.2 Optimization model based on the lexicographic criterion 23

2.3 Characterization of the ENSC value . . . . . . . . . . 25

3 Implementation of the $\alpha$-ENSC value 33

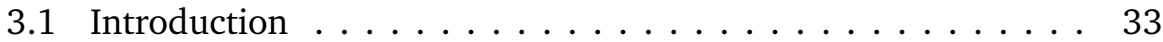

3.2 Procedural implementation of the $\alpha$-ENSC value . . . . . . . 36

3.3 The general $\alpha$-ENSC value . . . . . . . . . . . . . . 42

3.4 Optimization implementation of the $\alpha$-ENSC value . . . . . 45

3.5 Characterization of the $\alpha$-ENSC value . . . . . . . . . . 47

3.6 Bidding mechanism design for the $\alpha$-ENSC value . . . . . . . 51

4 Responsibility and sharing the cost of cleaning a polluted river 57

4.1 Introduction ...................... 57 
4.2 Preliminaries . . . . . . . . . . . . . . . . 60

4.3 Dynamic implementation of the sharing method and the $\alpha$ -

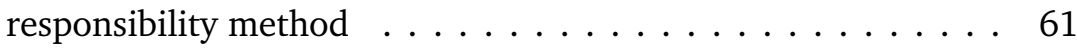

4.4 Characterization of the $\alpha$-responsibility method . . . . . . . 66 4.4.1 Characterization based on No Blind Cost property . . . 67 4.4.2 Characterization based on Upstream Symmetry . . . . 71

5 The general compromise value for cooperative games 75

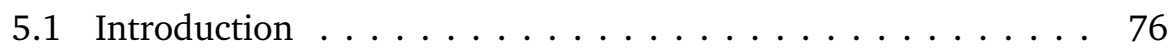

5.2 Preliminaries . . . . . . . . . . . . . . . 79

5.3 The general compromise value . . . . . . . . . . . 81

5.4 The general compromise value generated by sets with special

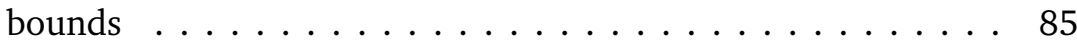

5.5 The general compromise value, the $\tau$-value, the $\chi$-value and

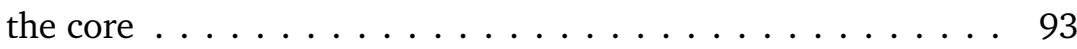

5.5.1 The general compromise value generated from the core 93

5.5.2 The relation between the general compromise value and the $\tau$-value . . . . . . . . . . . . . . 95

5.5.3 The relation between the general compromise value

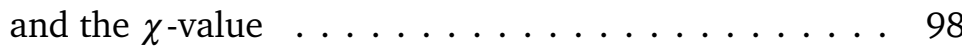

5.6 Characterization of the general compromise value . . . . . . 101

6 The optimal compromise values based on the lexicographic minimization criterion

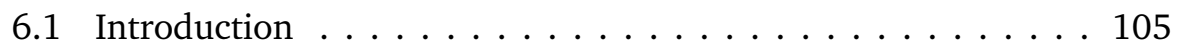

6.2 The optimal compromise value for cooperative games . . . . . . . . . . . . . . . . . 107

6.2.1 The determination of the optimal optimistic compromise value . . . . . . . . . . . . . . . . . . . . . 109

6.2.2 The determination of the optimal pessimistic compromise value . . . . . . . . . . . . . . . . . . . 111

6.2.3 The determination of the optimal average compromise value . . . . . . . . . . . . . . . . . . . 114

6.3 Characterization of the optimal compromise value . . . . . . 121 
6.3.1 Characterization of the optimal optimistic compromise value . . . . . . . . . . . . . . . . . . . 121

6.3.2 Characterization of the optimal pessimistic compromise value . . . . . . . . . . . . . . . . . . 123

7 Optimal solution concepts for cooperative games with stochastic payoffs

7.1 Introduction . . . . . . . . . . . . . . . . . . . . . . . . 129

7.2 Preliminaries . . . . . . . . . . . . . . . . . . . . 131

7.3 Optimization models for stochastic cooperative games based on coalitions . . . . . . . . . . . . . . . 133

7.3.1 The most stable solutions for stochastic cooperative games133

7.3.2 The fairest solution for stochastic cooperative games . . 136

7.4 Optimization models for stochastic cooperative games based on individual players . . . . . . . . . . . . . . 144

Summary

Bibliography

Acknowledgements 



\section{Chapter 1}

\section{Introduction}

\subsection{Game Theory}

One of the milestones in the studies of game theory is the publication of the classical book "Theory of games and economic behavior" [56], which has a far-reaching influence on the theory. In brief, game theory is the science of "strategy", a branch of mathematics that studies decision making situations and applies it to several fields, such as economics, computer science, political science, social science, or biology.

Game theory provides mathematical models for situations with conflicts and/or cooperation. Based on whether a binding agreement can be made among players, game theory consists of two branches: non-cooperative game theory and cooperative game theory. Given any decision making situation, conflict and/or cooperation occurs due to the interaction among individual decision makers (players). Interaction among players with different preferences may result in different potential payoffs for the players. Being rational decision makers, them will try to obtain his/her maximum possible payoff, while the other rational players will also try to find ways to maximize their payoffs. As we can see, game theory first puts situations with conflict and cooperation into mathematical models and then analyses these models. Roughly speaking, the study of game theory fall into two parts: the modelling part and the part of finding, designing a solution. 
The description of the mathematical models is composed of rules, the strategies for all players, potential payoffs, the preferences over the potential payoffs. Based on the rules, whether communication among players is allowed in order to make binding agreements, cooperative (allowed) and noncooperative (forbidden) game theory are distinguished. Von Neumann and Morgenstern [56] first introduced the fundamental model of game theory. Generally, mathematical models of game come, more or less, in three forms: the extensive (or tree) form, the normal (or strategy) form and the characteristic function (or coalitional) form.

As to the solution part, the solution concepts determine the resulting payoffs to players. It is notable that any solution concept is proposed based on some rules of fairness in view of the payoffs. Since there are so many criteria of fairness, various solution concepts have been investigated.

Throughout this work, we focus on cooperative game theory, more specifically the class of transferable utility games in characteristic function form (TU-games for short).

\subsection{Cooperative games}

Definition 1.1. A cooperative $n$-person game in characteristic function form is a pair $(N, v)$, where $N$ is the finite player set and $v: 2^{N} \rightarrow \mathbf{R}$ is the characteristic function on the set $2^{N}$ of all subsets of $N$ such that $v(\emptyset)=0$.

For any $S \subseteq N, v(S)$ is the worth that $S$ can earn by acting alone and the cardinality of $S$ is denoted by $s$. Let $\Gamma_{n}$ denote the class of all cooperative $n$-person games with player set $N$. The game space $\Gamma_{n}$ is in fact a Euclidean linear space. Throughout this work, we simply say game $v$ instead of $(N, v)$ if there is no confusion. For any pair $v, w \in \Gamma_{n}$ and $\alpha \in \mathbf{R}$, the $n$-person games $v+w$ and $\alpha v$ are defined as $(v+w)(S)=v(S)+w(S)$ and $(\alpha) v(S)=\alpha v(S)$ for all $S \subset N$. Given any $v \in \Gamma_{n}$, the dual game of $v$ is defined by $v_{*}(S)=$ $v(N)-v(N \backslash S)$ for all $S \subseteq N$.

Many economic situations can be analysed in the frame of the above model. In order to better understand the model, we first introduce a production economy with landowners and peasants, which has been studied both 
in [74] and [16].

Example 1.1. There are several peasants (without land) of the same type (w.r.t labour) denoted by $\{1, \cdots, m\}$ and one landowner denoted by $L$. If $t$ peasants are hired by the landowner, then they will produce a quantity of $f(t)$ of crop meassured in money, where $f:\{0, \cdots, m\} \rightarrow \mathbf{R}$ is the production function. We assume that the landowner by himself can not create anything, i.e., $f(0)=0$. Moreover, the function is non-decreasing.

This simple production situation can be modelled into a $(m+1)$-person game, of which the player set is $\{1, \cdots, m, L\}$ and its characteristic function $v$ assigning a worth or value $v(S)$ to every coalition $S$ is given by

$$
v(S)= \begin{cases}0 & \text { if } L \notin S \\ f(|S|-1) & \text { if } L \in S .\end{cases}
$$

The worth of any coalition without the landowner is equal to zero, since the peasants own no land. The worth of any coalition containing the landowner equals the monetary value that the peasants of this coalition create by cultivating the land of the landowner.

Apart from modelling the situations concerning profit, cooperative game theory also deals with situations concerning cost. Cost allocation problems first studied by means of game theory date back to 1942, the so-called Tennessee Valley Authority allocation problem [65]. This problem focuses on the cost allocation of the Wilson Dam and other reservoir projects. Next we will introduce another classical cost allocation problem called the airport game, which was first considered by Littlechild and Owen [46].

Example 1.2. In general, there are two kinds of costs for an airport. The first is the variable operating cost caused by the landings of different types of planes. The second is the fixed capital cost including various construction expenses. The landing costs can be directly charged from the corresponding planes (resp. the corresponding airlines). The main problem is how to allocate the fixed cost among planes. Notice that the construction of a runway depends on the largest plane using the airport. Such a problem can be written as the following cooperative game. 
Suppose there are $m$ types of planes. Denote planes of type $j$ as $N_{j}$, then the player (plane) set $N=\bigcup_{j=1}^{m} N_{j}$. Let $c_{i}$ be the cost that is sufficient for constructing a runway for planes of type $i$. We assume that the planes are ordered as $c_{1}<c_{2}<\cdots<c_{m}$. In this way, we could define the corresponding characteristic function as follows:

$$
v(S)=\max \left\{c_{j} \mid 1 \leqslant j \leqslant m, S \cap N_{j} \neq \emptyset\right\}, S \subseteq N .
$$

There are also many other situations that can be analysed by cooperative model, including the exchange economy with traders of different types (see [67] and [49]), the bankruptcy games [59] and the problem of sharing the cost of cleaning a polluted river [55].

Apart from those, Von Neumann and Morgenstern [56] applied game theory to describe a more abstract voting system, the so-called simple game.

Definition 1.2. An n-person game $v \in \Gamma_{n}$ is a simple game if it satisfies the following conditions:

$$
\begin{array}{ll}
v(S) \in\{0,1\} & \text { for all } S \subset N, \text { and } \\
v(S) \leqslant v(T)=1 \text { and, } & \text { for all } S \subset T \subset N .
\end{array}
$$

Coalitions with value 1 are called winning (or powerful) coalitions, and losing (or powerless) coalitions are those with value 0 . Among the class of simple games, unanimity games are very useful tool to analyse the property of solution concepts and have been broadly studied.

Definition 1.3. Given a finite player set $N$, for any $T \subset N, T \neq \emptyset$, the unanimity game $u_{T} \in \Gamma_{n}$ is defined as follows

$$
u_{T}(S)= \begin{cases}1 & \text { if } T \subseteq S \\ 0 & \text { otherwise }\end{cases}
$$

Given unanimity game $u_{T}$, any coalition is powerless if it doesn't include all the players of $T$, which implies that $T$ is exactly the set of veto players, whose absence results in losing coalitions. For any $T \subseteq N, T \neq \emptyset$, the standard 
game $b^{T} \in \Gamma_{n}$ is defined by

$$
b^{T}(S):= \begin{cases}1 & \text { if } S=T \\ 0 & \text { otherwise. }\end{cases}
$$

It turns out that both the sets $\left\{u_{T}\right\}_{T \in 2^{N} \backslash \emptyset}$ and $\left\{b^{T}\right\}_{T \in 2^{N} \backslash \emptyset}$ form a basis of the linear space $\Gamma_{n}$ (Note that every $n$-person game can be regarded as a vector of which the components are the worth of all coalitions).

\subsection{Solution concepts for cooperative games}

As mentioned earlier, cooperative game theory models situations of cooperation and it is widely used in many areas. The main subject of cooperative game theory is to determine an allocation rule which defines what portion of the societal benefit each participating player receives. Various allocation rules for cooperative games have been proposed concerning different fairness criteria. In this section, we introduce some classical solution concepts for cooperative games and these solutions will be further discussed in the following chapters.

The payoff vector in an $n$-person game is an $n$-dimensional vector $x \in$ $\mathbf{R}^{n}$ representing the amounts each player gets. $x_{i}$ represents the payoff to player $i$. One basic and a reasonable idea for such decision making problem is that we have to distribute exactly all the amount $v(N)$ to all players without surplus or deficit, which is called the efficiency principle. A pre-imputation is an efficient payoff, i.e., $x(N)=v(N)$, where $x(N)=\sum_{i \in N} x_{i}$. For any $v \in \Gamma_{n}$, denote the set of all pre-imputations as

$$
I^{*}(v)=\left\{x \in \mathbf{R}^{n} \mid x(N)=v(N)\right\} .
$$

As a rational decision maker, everyone wants to benefit from the cooperation with others, which requires that every individual player expects to obtain at least the worth that he can earn by acting alone. Such expectation is called individual rationality, i.e., $x_{i} \geqslant v(i)$ for any payoff vector $x$ and player $i \in N^{1}$.

\footnotetext{
${ }^{1}$ As usual, we define $\mathrm{v}(\mathrm{i}):=\mathrm{v}(\{\mathrm{i}\})$.
} 
A pre-imputation is an imputation if it satisfies individual rationality. For any $v \in \Gamma_{n}$, denote the set of all imputations as

$$
I(v)=\left\{x \in \mathbf{R}^{n} \mid x(N)=v(N) \text { and } x_{i} \geqslant v(i), \forall i \in N\right\} .
$$

Clearly, the imputation set is non-empty iff $\sum_{i \in N} v(i) \leqslant v(N)$. Formally a solution (or value) $\varphi: \Gamma_{n} \rightarrow \mathbf{R}^{n}$ is a function that maps every $v \in \Gamma_{n}$ to a set of payoff vectors. One of the most significant one-point solution concepts in literature is the Shapley value which was proposed and characterized by Shapley in 1953 [72].

Definition 1.4. For any $v \in \Gamma_{n}$ and $i \in N$, the Shapley value is given by

$$
\phi_{i}(v)=\sum_{S \subset N \backslash\{i\}} \frac{s !(n-s-1) !}{n !}[v(S \cup\{i\})-v(S)] .
$$

In fact the factors $\left\{\frac{s !(n-s-1) !}{n !}\right\}_{s=0}^{n-1}$ can be viewed as a probability distribution over all coalitions not containing player $i$ since $\sum_{S \subset N \backslash\{i\}} \frac{s !(n-s-1) !}{n !}$ equals 1. Coalitions with the same size have identical probability. Therefore, the Shapley value assigns the expected marginal contributions over all permutations to all the players.

Instead of taking all the coalitions into account as the Shapley value does, Moulin [53] introduced the equal allocation of non-separable costs value (ENSC value).

Definition 1.5. For any $v \in \Gamma_{n}$, the ENSC value allocates to each player $i$ his marginal contribution to the worth of the grand coalition $b_{i}^{v}=v(N)-v(N \backslash i)$ and then evenly divides the remaining benefit among all players, i.e.,

$$
\operatorname{ENSC}_{i}(v):=b_{i}^{v}+\frac{1}{n}\left[v(N)-\sum_{j \in N} b_{j}^{v}\right], \forall i \in N .
$$

For simplicity, we call $b_{i}^{v}$ the grand marginal contribution for player $i$ in game $v$. In the context of cost allocation problems, $b_{j}^{v}$ is called the separable cost of player $j$. Separable cost is the change in cost of a given project 
when player $j$ joins the formed coalition $N \backslash\{j\}$. Thus this cost can be directly charged to corresponding player. The remaining part $v(N)-b^{v}(N)$ is called the non-separable cost. Different ways to allocate the non-separable cost generate various allocation methods. As we can see, the ENSC value allocates the non-separable cost equally among the players. Without going into details, we mention two other famous allocation methods including the ACA-method and the SCRB-method [103], which were both introduced to deal with water resource allocation.

Driessen and Funaki [24] introduced a more straightforward value called the center-of-gravity of imputation set value (the CIS value for short).The CIS value is a solution that only concerns the worths of the individuals and the grand coalition. Formally the definition is as follows.

Definition 1.6. For any $v \in \Gamma_{n}$, the CIS value gives every player its individual worth, and then distributes the remaining worth of the grand coalition equally among all players, i.e.,

$$
\operatorname{CIS}_{i}(v)=v(i)+\frac{1}{n}\left[v(N)-\sum_{j \in N} v(j)\right], \forall i \in N .
$$

Another natural solution concept is the equal division value (the ED value), which allocates the total worth of the grand coalition equally among the players.

Definition 1.7. For any $v \in \Gamma_{n}$ and player $i \in N$, the ED value is defined as

$$
E D_{i}(v)=\frac{v(N)}{n}, \forall i \in N .
$$

Several solution concepts for cooperative games have been studied based on the concept of the excess [19], which is the gap between the worth of a coalition and what it can obtain from the proposed payoff.

Definition 1.8. For any $v \in \Gamma_{n}$ and a payoff vector $x \in \mathbf{R}$, the excess of a coalition $S \subset N$ is given by

$$
e^{v}(S, x)=v(S)-x(S) .
$$


The notion of excess usually is taken as a measurement of complaints for coalitions towards a given payoff. A nonnegative (nonpositive respectively) excess of coalition $S$ at the payoff vector $x$ in the game $v$ represents the gain (loss) to the coalition $S$ if its members withdraw from the grand coalition, offering payoff vector $x$, in order to form their own coalition.

Gillies proposed a set-valued solution concept, namely the core [29], as a set of efficient payoff vectors with non-positive excesses.

Definition 1.9. For any $v \in \Gamma_{n}$, the core of game $v$ is defined by

$$
C(v)=\left\{x \in \mathbf{R}^{n} \mid x(N)=v(N) \text { and } e^{v}(S, x) \leqslant 0 \text { for all } S \subset N\right\} .
$$

For any payoff vector in the core of a cooperative game (if the core is not empty), it implies that no coalition has a worth greater than the sum of its members' payoffs. Therefore, no coalition has the incentive to leave the grand coalition in order ro receive a larger payoff. Such a payoff imputation has an inherent stability. Shapley and Shubik revealed that the core of a cooperative game exists if and only if the game is balanced [74].

The nucleolus, introduced by Schmeidler [71], is the outcome of a lexicographic minimization procedure over the excess vectors which are associated with allocations. Given any $v \in \Gamma_{n}$ and $x \in \mathbf{R}^{n}$, let $\theta(x)$ be a $2^{n}$ tuple whose components are the excesses $e^{v}(S, x)$ arranged in non-increasing order. The Nucleolus is the only allocation rule that lexicographically minimizes the complaints among all coalitions over the imputation set.

Definition 1.10. The nucleolus of a cooperative game $v \in \Gamma_{n}$ is the set of all imputations $x \in I(v)$ satisfying

$$
\theta(x) \leq_{L} \theta(y), \quad \forall y \in I(v)
$$

where $\leq_{L}$ represents the lexicographic order.

Instead of pushing down the highest excess, Ruiz et al. [68-70] introduced the least square values as the unique minimizer of the variance of the total excesses for coalitions. They consider the following problem for a given game 
$v \in \Gamma_{n}:$

$$
\begin{gathered}
\text { Minimize } \sum_{S \in 2^{N} \backslash \emptyset}(v(S)-x(S))^{2} \\
\text { s.t. } x \in I^{*}(v) .
\end{gathered}
$$

The optimal solution of this problem is defined as the least square pre-nucleolus [68], that is

$$
\lambda_{i}(v)=\frac{v(N)}{n}+\frac{1}{n 2^{n-2}}\left[n a_{i}(v)-\sum_{j \in N} a_{j}(v)\right], \forall i \in N,
$$

where $a_{i}(v)=\sum_{S \ni i} v(S)$.

\subsection{Overview}

In a cooperative situation, every individual decision-maker certainly has his or her own expectation that reflects how much he/she seeks to obtain from the cooperation with others. But in reality, it is not always possible to allocate such ideal payoff to every player, leading to the so-called complaint for players. Given an allocation scheme, players' decisions will highly depend on whether the proposed allocation minimizes the corresponding complaint for them. In other words, a fair and reasonable solution should minimize players, complaint under a certain criterion. As we have mentioned in the previous section, various solution concepts have been proposed based on the concept of complaint, i.e., the excess. The core is defined as the pre-imputation with nonpositive complaint. The (pre)nucleolus minimizes the maximal complaint under lexicographic criterion. The least square values are solution concepts that minimize the total complaint under the least square criterion. We can see that all those solution concepts are proposed based on the implicit assumption that coalitions view the worth obtained by acting alone as their ideal payoff. Such assumption in some sense ignore the cooperation effect among players. This thesis will further explore solution concepts by investigating different complaints for players. 
In Chapter 2, we implement the ENSC value by considering the complaint for individual players. Recall that the grand marginal contribution for every player is an upper bound for the core of the game, therefore players may view it as their ideal payoff. We analyse the solution that minimizes the corresponding total complaint for individual players under the least square criterion. Inspired by the work of Malawski [47], we also study the dynamic formation of the grand coalition. We assume that players enter the game one by one and every new entrant will obtain his or her grand marginal contribution. Under this procedure, we define the compromised ideal payoff for players. Interestingly, we could also implement the ENSC value by minimizing the total complaint under both the least square and lexicographic criterion.

Furthermore, in Chapter 3, we generalize the model in Chapter 2. Players who want to obtain all his or her grand marginal contribution are totally egoistic, which is quite an extreme situation. Therefore we introduce a parameter which measures the egoism for players. Following the same lines as in Chapter 2, we obtain and characterize a new solution concept, namely the $\alpha$-ENSC value.

In Chapter 4, we study the cost allocation problem of cleaning a polluted river, which is an application of the method discussed in Chapters 2 and 3. Firms or factories along a river should undertake the responsibility of the pollution induced by them. Basically, this is a cost allocation problem. Agents from different areas (upstream and downstream) make different contributions to the pollutant due to the special structure of the river. Ni and Wang proposed the Local responsibility sharing method and Upstream equal sharing method according to two famous precepts, "Absolute territorial sovereignty" and "Unlimited territorial integrity", respectively. Although these two methods have many good properties, they ignore the flow of the pollution from upstream to downstream. In our work, we take into account the water flow and study the formation of the agents. It turns out that the final allocation is the combination of the Local responsibility sharing method and Upstream equal sharing method. Furthermore, we provide two kinds of characterizations of the new method based on the no blind cost property and upstream symmetry respectively.

In Chapter 5, we also explore solution concepts by discussing the concept 
of ideal payoff in a combinatorial way. We not only consider the ideal payoff but also the minimal payoff for players. Given a set of potential payoffs, we could obtain the upper and lower bounds for players' payoffs. Generally, it will violate the efficiency principle if we allocate the payoff according to the upper and lower vector. Therefore players have to make concessions with respect to the ideal payoff. In this way, we define the so-called general compromise value, which is the unique pre-imputation lying on the segment between the maximal and minimal potential payoffs. We show that the general compromise value generalizes many classical solution concepts, such as the $\tau$-value and the $\chi$-value, by considering different set of potential payoffs.

In Chapter 6, we study the optimal compromise values based on the lexicographic criterion. Instead of considering the classical concept of excess, we define two kinds (optimistic and pessimistic) of concession/compliance for coalitions. In the optimistic case, coalitions view the sum of the grand marginal contributions of all its member as its ideal payoff. In the pessimistic term, coalitions will measure their complaint from its complementary coalition assuming that the others will only obtain their individual worth. We can prove that the optimal solution concepts that minimize these two corresponding complaint vector under the lexicographic criterion coincides with the ENSC value and the CIS value respectively. More interestingly, if we consider the corresponding per capita complaint, the same optimal solution concepts can be derived. Moreover, we provide characterizations of these two solutions by means of equal optimistic (pessimistic) maximal complaint property, which is inspired by the definition of the prekernel.

In Chapter 7, we study solution concepts for cooperative games with stochastic payoffs by discussing different optimization models. We define the most stable solutions by minimizing the total variance of excesses of all coalitions. Note that these most stable solutions constitute a set of values, which seems less satisfying. Among the set of most stable solutions, we select an unique solution of which the excesses of coalitions are closest to the average excess. All of these optimal models are under the least square criterion, both from the perspective of coalitions and individual players. We also propose the expected and variance nucleolus for cooperative games with stochastic payoffs, and relations between these solutions are exhibited. 



\section{Chapter 2}

\section{Implementation of the ENSC value}

This chapter devotes to the study of the egalitarian non-separable contribution value (ENSC value) for cooperative games. On the one hand, we show that the ENSC value is the unique optimal solution that minimizes the total complaints for individual players over the pre-imputation set. On the other hand, analogous to the way of determining the nucleolus, we obtain the ENSC value by applying the lexicographic order over the individual complaints. Moreover, we offer alternative characterizations of the ENSC value by proposing several new properties such as dual nullifying player property, dual dummifying player property and grand marginal contribution monotonicity.

\subsection{Introduction}

Recall that cooperative game theory deals with the mathematical models of cooperative situations in which the grand coalition forms. Thus every single player plays a significant role in the formation of the grand coalition, suggesting that the ideal payoff for players is the marginal contribution to the worth of the grand coalition. Given any allocation, there always exists a gap 
between the ideal payoff and the proposed allocation, resulting in the complaints of the players. With the same method applied in the determination of the least square value, we select the unique payoff vector that minimizes the summation of the variance of the total complaints for individual players. It will be shown that the optimal solution coincides with the ENSC value.

Inspired by the interpretation of the Shapley value ( [72] [11] [47]), an alternative ideal payoff for individual players is proposed. Suppose that players join the game one by one and every new entrant charges the marginal contribution he creates to the formed coalition as his payoff, then the Shapley value is defined by averaging the marginal vectors over all possible orders. The Shapley value can be treated as an egoistic allocation in the sense that every new entrant takes his entire marginal contribution for himself. In this chapter, we deal with the situation in which players are totally egoistic. That is, every new entrant claims his grand marginal contribution rather than the marginal contribution of the formed coalition and what's left is shared equally among the preceding players. Notice that in the formation of the grand coalition, every player obtains his grand marginal contribution as a new entrant but also has to undertake a portion of gaps generated by his successors, yielding the so-called compromised ideal payoff. In this chapter, it will be proved that the ENSC value is the unique pre-imputation that minimizes the summation of the variance of the total compromised complaints for individual players. By analyzing the involved procedure, we could conclude that the ENSC value is a more egoistic value than the Shapley value. In fact, Malawski [47] elaborated that the equal division value is more altruistic than the solidarity value [57] by proposing the so-called "Procedural" value. And the Shapley value is in some sense egoistic compared to the solidarity value, while our work reveals the fact that the ENSC value is more egoistic than the Shapley value. Moreover, analogously to the way of obtaining the Nucleolus, we implement the ENSC value by lexicographically minimizing the individual excess vector over the pre-imputation set. From the perspective of optimization, the conclusion indicates a coincidence between these two values, which was first studied by Driessen and Funaki [24].

There exists a lot of literature concentrating on the characterization of 
the ENSC value. For instance, Xu [99] and Hwang [101] both characterized the ENSC value with the aid of associated consistency, which was inspired by Hamiache's [36] characterization of the Shapley value. The associated consistency of an allocation rule implies that it gives the same payoff to each participating agent in the original game and in the imaginary associated game, which reflects the expectations elaborated by the agents. Ju and Wettstein [42] characterized the ENSC value as the implementation result of a bidding mechanism through non-cooperative viewpoint. In this chapter, we provide two alternative characterizations of the ENSC value: the first as the unique solution to an optimization problem, the second as the unique satisfier of a particular collection of axioms. Several new properties are proposed including the dual nullifying player property, the dual dummifying player property and grand marginal contribution monotonicity. The dual nullifying player property and dual dummifying player property are inspired by the concepts of the nullifying and dummifying player property, which were introduced by van den Brink [88] and Casajus [12] respectively. The dual nullifying player property states that any player that brings nullifying influence to coalitions containing him through an indirect way will get nothing. The dual dummifying player property guarantees the grand marginal contribution for players who carry out dummifying influence to coalitions. In addition, grand marginal contribution monotonicity ensures that players with a larger grand marginal contribution can obtain a larger portion of the total benefit.

\subsection{Optimization implementation of the ENSC value}

\subsubsection{Optimization model based on the least square criterion}

Ruiz et al. [68] defined the least square pre-nucleolus as the unique preimputation that minimizes the variance of the excess of coalitions. Tijs [81] revealed that the grand marginal contribution $b^{v} \in \mathbf{R}^{n}$ is an upper bound for the core of game $v \in \Gamma_{n}$. The core, introduced by Gillies [29], is the set of all imputations that can not be improved upon by any coalition, that is, the core is always internally stable. Based on this fact, any player $i \in N$ in a game $v$ will regard $b_{i}^{v}$ as his ideal payoff and certainly prefer an allocation which is 
closest to the vector $b^{v}$. Inspired by Ruiz, our aim is to select a payoff vector that has the least Euclidean distance to the marginal contribution vector $b^{v}$. The following optimization problem is taken into account.

Problem 2.1.

$$
\begin{aligned}
& \min \sum_{i \in N}\left(b_{i}^{v}-x_{i}\right)^{2} \\
& \text { s.t. } \quad x \in \mathbf{R}^{n} \text { and } \sum_{i \in N} x_{i}=v(N) .
\end{aligned}
$$

Theorem 2.1. For any $v \in \Gamma_{n}$, there exists a unique solution $x^{*}$ of Problem 2.1, which coincides with the ENSC value, i.e.,

$$
x_{i}^{*}=b_{i}^{v}+\frac{1}{n}\left[v(N)-\sum_{j \in N} b_{j}^{v}\right], \quad \forall i \in N .
$$

Proof. The solution minimizes the Euclidean distance between $b^{v}$ and the set of point in the efficient hyperplane. It is therefore an orthogonal projection of $b^{v}$ on the efficient hyperplane, hence it is unique.. Thus, in order to find the optimal solution, it is necessary and sufficient to verify the Lagrange conditions. The Lagrange function of Problem 2.1 is

$$
L(x, \lambda)=\sum_{i \in N}\left(b_{i}^{v}-x_{i}\right)^{2}+\lambda\left(\sum_{i \in N} x_{i}-v(N)\right) .
$$

The Lagrange conditions are then

$$
L_{x_{i}}(x, \lambda)=2\left(x_{i}^{*}-b_{i}^{v}\right)+\lambda=0, \quad i \in N .
$$

Therefore, it holds that

$$
x_{i}^{*}-b_{i}^{v}=x_{j}^{*}-b_{j}^{v}, \quad \forall i, j \in N .
$$


Furthermore, by the constraint equation $\sum_{i \in N} x_{i}=v(N)$, the solution for the equations in (2.4) is

$$
x_{i}^{*}=b_{i}^{v}+\frac{1}{n}\left[v(N)-\sum_{j \in N} b_{j}^{v}\right]=\operatorname{ENSC}_{i}(v) .
$$

Remark 2.1. One may reasonably deduce that if every player aspires to get the worth of the grand coalition $v(N)$, then the gap between the worth of the grand coalition and the proposed payoff can be regarded as complaints for players. By minimizing $\sum_{i \in N}\left(v(N)-x_{i}\right)^{2}, x \in \mathbf{R}^{n}$ over the pre-imputation set, one could find that projecting the vector consisting of equal components on the efficient hyperplane yields the equal division value.

As stated in the Introduction, the Shapley value is in some sense an egoistic value which assigns to every new entrant his marginal contribution to the formed coalition. Here we deal with the situation where players are totally egoistic, that is, all the newcomers will charge the grand marginal contributions as their ideal payoffs.

Definition 2.1. For any $v \in \Gamma_{n}$ and $\pi \in \Pi(N)$, where $\Pi(N)$ denotes the set of all permutations on $N$, a player $i \in N$ is totally egoistic if he claims $C_{i}^{\pi}$ when he joins the game, where

$$
C_{i}^{\pi}:= \begin{cases}v(i), & \text { if } \pi(i)=1, \\ b_{i}^{v}, & \text { otherwise. }\end{cases}
$$

The gap $v\left(S_{\pi}^{i}\right)-v\left(S_{\pi}^{i} \backslash i\right)-C_{i}^{\pi}$ generated by player $i$ is shared among the players. The portion for player $k \in N$ is $G_{i k}^{\pi}$, i.e.,

$$
G_{i k}^{\pi}:= \begin{cases}\frac{v\left(S_{\pi}^{i}\right)-v\left(S_{\pi}^{i} \backslash i\right)-C_{i}^{\pi}}{\pi(i)-1}, & \text { if } k \in S_{\pi}^{i} \backslash\{i\}, \\ 0, & \text { otherwise. }\end{cases}
$$

Here $S_{\pi}^{i}:=\{j \in N: \pi(j) \leq \pi(i)\}$ denotes the set that consists of player $i$ and all his predecessors under permutation $\pi$ and $\pi(i)$ is the position of player $i$ in order $\pi$. 
For any dynamic coalitional formation order, a totally egoistic player will claim his grand marginal contribution, while what's left is equally shared among the preceding participants. Particularly, a player who takes up the first position only obtains his individual value since there are no predecessors.

Example 2.1. In order to illustrate the above procedure, we consider the following three-person game $v \in \Gamma_{n}$ under the assumption that all players are totally egoistic and the dynamic coalitional formation order is $(3,1,2)$.

At the beginning, player 3 joins the game. As the sole present player, player 3 obtains his individual worth $v(3)$, while player 1 and 2 obtain nothing at the moment. The payoff for players in this stage is shown in Table 1.

TABLE 2.1 Payoff for players when 3 joins.

\begin{tabular}{lccc}
\hline Player & $\mathbf{1}$ & $\mathbf{2}$ & $\mathbf{3}$ \\
\hline Allocation & 0 & 0 & $v(3)$ \\
\hline
\end{tabular}

Then player 1 joins, he obtains his grand marginal contribution $b_{1}^{v}$. The only preceding player 3 achieves an extra payoff, the amount of which equals to the new entrant's marginal contribution to the preceding player 3 minus his grand marginal contribution, i.e., $v(1,3)-v(3)-b_{1}^{v}$. The payoff for players in this stage is shown in Table 2.

TABLE 2.2 Payoff for players when 1 joins.

\begin{tabular}{lccc}
\hline Player & $\mathbf{1}$ & $\mathbf{2}$ & $\mathbf{3}$ \\
\hline Allocation & $b_{1}^{v}$ & 0 & $v(1,3)-v(3)-b_{1}^{v}$ \\
\hline
\end{tabular}

Finally player 2 joins and obtains $b_{2}^{v}$. The remaining $v(N)-v(1,3)-b_{2}^{v}$ is shared equally among the preceding players 1 and 3 . The payoff for players in the final stage is shown in Table 3.

By summing the payoffs in these three stages, we get the final payoff as shown in Table 4. 
TABLE 2.3 Payoff for players when 2 joins.

\begin{tabular}{lccc}
\hline Player & $\mathbf{1}$ & $\mathbf{2}$ & $\mathbf{3}$ \\
\hline \multirow{2}{*}{ Allocation } & $\frac{v(N)-v(1,3)-b_{2}^{v}}{2}$ & $b_{2}^{v}$ & $\frac{v(N)-v(1,3)-b_{2}^{v}}{2}$ \\
\hline
\end{tabular}

TABLE 2.4 Payoff for players under under formation procedure $\pi=(3,1,2)$

\begin{tabular}{lcccc}
\hline Player $\backslash$ Step & $\mathbf{1}$ & $\mathbf{2}$ & $\mathbf{3}$ & Player's final payoff \\
\hline $\mathbf{3}$ & $v(3)$ & $v(1,3)-v(3)-b_{1}^{v}$ & $\frac{v(N)-v(1,3)-b_{2}^{v}}{2}$ & $-b_{1}^{v}+\frac{v(N)+v(1,3)-b_{2}^{v}}{2}$ \\
$\mathbf{1}$ & 0 & $b_{1}^{v}$ & $\frac{v(N)-v(1,3)-b_{2}^{v}}{2}$ & $b_{1}^{v}+\frac{v(N)-v(1,3)-b_{2}^{v}}{2}$ \\
$\mathbf{2}$ & 0 & 0 & $b_{2}^{v}$ & $b_{2}^{v}$ \\
\hline
\end{tabular}

In the formation of the grand coalition, each new entrant first obtains his grand marginal contribution and then bears the gaps generated by his successors, which somehow reflects a compromise between the ideal payoff $b^{v}$ and the gaps. As shown in the above example, the final payoff for player 1 consists of two parts, his ideal payoff $b_{1}^{v}$ and the compromise part $\frac{v(N)-v(1,3)-b_{2}^{v}}{2}$ generated by his successor 2 .

Definition 2.2. For any $v \in \Gamma_{n}$ and $\pi \in \Pi(N)$, let all players be totally egoistic. The compromised ideal payoff for player $i$ under coalitional formation order $\pi$ is defined as

$$
\eta_{i}^{v \pi}:= \begin{cases}v(i)+\sum_{k=\pi^{-1}(i)+1}^{n} \frac{v\left(S_{\pi}^{\pi^{-1}(k)}\right)-v\left(S_{\pi}^{\pi^{-1}(k)} \backslash \pi(k)\right)-b_{\pi(k)}^{v}}{k-1}, & \text { if } \pi(i)=1, \\ b_{i}^{v}+\sum_{k=\pi^{-1}(i)+1}^{n} \frac{v\left(S_{\pi}^{\pi^{-1}(k)}\right)-v\left(v \left(S_{\pi}^{\pi^{-1}(k)} \backslash \pi(k)-b_{\pi(k)}^{v}\right.\right.}{k-1}, & \pi(i) \neq 1, n, \\ b_{i}^{v}, & \pi(i) \neq n,\end{cases}
$$

where $\pi^{-1}(k)$ denotes the player who possesses position $k$ in order $\pi$.

Definition 2.3. For any $v \in \Gamma_{n}$ and $\pi \in \Pi(N)$, let all players be totally egoistic. The compromised complaint of player $i$ towards the payoff vector $x \in \mathbf{R}^{n}$ with 
respect to the compromised ideal payoff $\eta_{i}^{v \pi}$ is

$$
e\left(i, x, \eta^{v \pi}\right):=\eta_{i}^{v \pi}-x_{i}, i \in N \text {. }
$$

The expression $e\left(i, x, \eta^{v \pi}\right)$ indicates the gap between player's compromised ideal payoff and the proposed payoff. The larger $e\left(i, x, \eta^{v \pi}\right)$ is, the more dissatisfied player $i$ feels. By minimizing the summation of the variance of the compromised complaints over the pre-imputation set, a unique optimal solution is obtained. This solution can be seen as the least unsatisfied payoff by the fact that it is the vector closest to the compromised ideal payoff vector under Euclidean distance. Formally, the following optimization problem is considered.

\section{Problem 2.2.}

$$
\begin{aligned}
& \min \sum_{i \in N} \sum_{\pi \in \Pi(N)} e^{2}\left(i, x, \eta^{v \pi}\right) \\
& \text { s.t. } \quad x \in \mathbf{R}^{n} \text { and } x(N)=v(N) .
\end{aligned}
$$

Theorem 2.2. For any $v \in \Gamma_{n}$, there exists a unique optimal solution $x^{*}$ for Problem 2.2, which coincides with the ENSC value.

In order to show the validity of Theorem 2.2 , the following lemma is taken into account.

Lemma 2.3. For any game $v \in \Gamma_{n}$, the average compromised ideal payoff coincides with the ENSC value, i.e.,

$$
\frac{1}{n !} \sum_{\pi \in \Pi(N)} \eta_{i}^{v \pi}=\operatorname{ENSC}_{i}(v)
$$

where $\eta_{i}^{v \pi}$ is defined as in (2.8).

Proof. For notional convenience, $v\left(S_{\pi}^{\pi^{-1}(k)}\right)-v\left(S_{\pi}^{\pi^{-1}(k)} \backslash \pi^{-1}(k)\right)$ is denoted by $m_{\pi(k)}$, therefore we have

$$
\frac{1}{n !} \sum_{\pi \in \Pi(N)} \eta_{i}^{v \pi}=\frac{1}{n} b_{i}^{v}+\sum_{\pi: \pi(i)=1} \frac{1}{n !}\left[v(i)+\sum_{k=\pi(i)+1}^{n} \frac{m_{\pi(k)}-b_{\pi^{-1}(k)}^{v}}{k-1}\right]
$$




$$
\begin{aligned}
& +\sum_{\pi: \pi(i) \neq 1, n} \frac{1}{n !}\left[b_{i}^{v}+\sum_{k=\pi(i)+1}^{n} \frac{m_{\pi(k)}-b_{\pi^{-1}(k)}^{v}}{k-1}\right] \\
& =\frac{1}{n} b_{i}^{v}+\frac{(n-1) !}{n !} v(i)+\sum_{\pi: \pi(i)=1} \frac{1}{n !} \sum_{k=\pi(i)+1}^{n} \frac{m_{\pi(k)}-b_{\pi^{-1}(k)}^{v}}{k-1} \\
& +\frac{(n-2)(n-1) !}{n !} b_{i}^{v}+\sum_{\pi: \pi(i) \neq 1, n} \frac{1}{n !} \sum_{k=\pi(i)+1}^{n} \frac{m_{\pi(k)}-b_{\pi^{-1}(k)}^{v}}{k-1} \\
& =\frac{1}{n} v(i)+\frac{n-1}{n} b_{i}^{v}+\sum_{\pi(i) \neq n} \frac{1}{n !} \sum_{k=\pi(i)+1}^{n} \frac{m_{\pi(k)}-b_{\pi^{-1}(k)}^{v}}{k-1} \\
& =\frac{1}{n} v(i)+\frac{n-1}{n} b_{i}^{v}+\sum_{l \in N \backslash\{i\}} \sum_{\pi: S_{\pi}^{l} \ni i} \frac{1}{n !} \frac{\left(v\left(S_{\pi}^{l}\right)-v\left(S_{\pi}^{l} \backslash l\right)\right)-b_{l}^{v}}{\left|S_{\pi}^{l}\right|-1} \\
& =\frac{1}{n} v(i)+\frac{n-1}{n} b_{i}^{v}+\sum_{l \in N \backslash\{i\}} \sum_{S \ni i, l} \frac{(v(S)-v(S \backslash l))-b_{l}^{v}}{s-1} \cdot \frac{(s-1) !(n-s) !}{n !} \\
& =\frac{1}{n} v(i)+\frac{n-1}{n} b_{i}^{v}+\sum_{l \in N \backslash\{i\}} \sum_{S \ni i, l}\left[(v(S)-v(S \backslash l))-b_{l}^{v}\right] \cdot \frac{(s-2) !(n-s) !}{n !} \\
& =\frac{1}{n} v(i)+\frac{n-1}{n} b_{i}^{v}+\sum_{l \in N \backslash\{i\}} \sum_{S \ni i, l} v(S) \cdot \frac{(s-2) !(n-s) !}{n !} \\
& -\sum_{l \in N \backslash\{i\}} \sum_{S \ni i, l} v(S \backslash l) \cdot \frac{(s-2) !(n-s) !}{n !}-\sum_{l \in N \backslash\{i\}} \sum_{S \ni i, l} b_{l} \cdot \frac{(s-2) !(n-s) !}{n !} \\
& =\frac{1}{n} v(i)+\frac{n-1}{n} b_{i}^{v}+\sum_{S \ni i,|S| \geq 2} \sum_{l \in S \backslash\{i\}} v(S) \cdot \frac{(s-2) !(n-s) !}{n !} \\
& -\sum_{l \in N \backslash\{i\}} \sum_{T \subseteq N \backslash l, T \ni i} v(T) \cdot \frac{(t+1-2) !(n-(t+1)) !}{n !}-\sum_{l \in N \backslash\{i\}} \sum_{S \ni i, l} b_{l}^{v} \cdot \frac{(s-2) !(n-s) !}{n !} \\
& =\frac{1}{n} v(i)+\frac{n-1}{n} b_{i}^{v}+\sum_{S \ni i,|S| \geq 2}(s-1) v(S) \cdot \frac{(s-2) !(n-s) !}{n !} \\
& \text { - } \sum_{T \ni i,|T| \leq n-1} \sum_{l \notin T} v(T) \cdot \frac{(t-1) !(n-t-1) !}{n !}-\sum_{l \in N \backslash\{i\}} \sum_{s=2}^{n} b_{l}^{v} \cdot \frac{(s-2) !(n-s) !}{n !}\left(\begin{array}{c}
n-2 \\
s-2
\end{array}\right) \\
& =\frac{1}{n} v(i)+\frac{n-1}{n} b_{i}^{v}+\sum_{S \ni i,|S| \geq 2} v(S) \cdot \frac{(s-1) !(n-s) !}{n !}
\end{aligned}
$$




$$
\begin{aligned}
& -\sum_{T \ni i,|T| \leq n-1}(n-t) v(T) \cdot \frac{(t-1) !(n-t-1) !}{n !}-\sum_{l \in N \backslash\{i\}} \sum_{s=2}^{n} b_{l}^{v} \cdot \frac{1}{n(n-1)} \\
& =\frac{1}{n} v(i)+\frac{n-1}{n} b_{i}^{v}+\sum_{S \ni i,|S| \geq 2} v(S) \cdot \frac{(s-1) !(n-s) !}{n !} \\
& -\sum_{T \ni i,|T| \leq n-1} v(T) \cdot \frac{(t-1) !(n-t) !}{n !}-\sum_{l \in N \backslash\{i\}} \frac{b_{l}^{v}}{n} \\
& =\frac{1}{n} v(i)+\frac{n-1}{n} b_{i}^{v}-\frac{1}{n} v(i)+\frac{v(N)}{n}-\sum_{l \in N \backslash\{i\}} \frac{b_{l}^{v}}{n} \\
& =b_{i}^{v}+\frac{v(N)-\sum_{l \in N} b_{l}^{v}}{n} \\
& =E_{N S C_{i}(v)}
\end{aligned}
$$

Proof of Theorem 2.2. The objective function and the feasible set of Problem 2.2 are both convex, hence there is only one optimal solution if it exists. It is necessary and sufficient to verify the Lagrange conditions so as to find the optimal solution. Formally, the Lagrange function of Problem 2.2 is

$$
L(x, \lambda)=\sum_{i \in N} \sum_{\pi \in \Pi(N)}\left(\eta_{i}^{v \pi}-x_{i}\right)^{2}+\lambda\left(\sum_{i \in N} x_{i}-v(N)\right) .
$$

Taking the derivative of this function, the Lagrange conditions are obtained as

$$
L_{x_{i}}(x, \lambda)=-2 \sum_{\pi \in \Pi(N)}\left(\eta_{i}^{v \pi}-x_{i}\right)+\lambda=0, \quad \forall i \in N .
$$

Thus for all $i, j \in N$, we have

$$
\sum_{\pi \in \Pi(N)}\left(\eta_{i}^{v \pi}-x_{i}\right)=\sum_{\pi \in \Pi(N)}\left(\eta_{j}^{v \pi}-x_{j}\right) .
$$

By Lemma 2.3 and the constraint equation $\sum_{i \in N} x_{i}=v(N)$, we straightforward to obtain that the optimal solution $x^{*}$ is

$$
x_{i}^{*}=\frac{1}{n !} \sum_{\pi \in \Pi(N)} \eta_{i}^{v \pi}=\operatorname{ENSC}_{i}(v) .
$$




\subsubsection{Optimization model based on the lexicographic criterion}

The former optimization problems both characterize the ENSC value as the unique allocation rule that minimizes the total complaints for individual players under the least square criterion. We now turn to explore the optimal solution that pushes down the maximal complaints for individual players under the lexicographic criterion, which is similar to the method of obtaining the nucleolus. Instead of considering the complaints for coalitions, we select the pre-imputation that minimizes the maximal average compromised complaint for the individual players. For any $x \in I^{*}(v)$, let $\theta^{*}(x)$ be the $n$ tuple whose components are the average compromised complaint, i.e., $\theta_{i}^{*}(x)=$ $\frac{1}{n !} \sum_{\pi \in \Pi(N)} e\left(i, x, \eta^{v \pi}\right)$, arranged in non-increasing order. The next theorem states that the ENSC value is the unique pre-imputation that lexicographically minimizes the average compromised complaint vector $\theta^{*}(x)$ over $I^{*}(v)$.

Theorem 2.4. For any $v \in \Gamma_{n}$, the ENSC value is the unique pre-imputation $x \in I^{*}(v)$ satisfying

$$
\theta^{*}(x) \leq_{L} \theta^{*}(y), \quad \forall y \in I^{*}(v)
$$

Proof. Given any $v \in \Gamma_{n}$, let $x$ be the pre-imputation satisfying

$$
\theta^{*}(x) \leq_{L} \theta^{*}(y), \quad \forall y \in I^{*}(v) .
$$

Now we assert that $\theta_{i}^{*}(x)=\theta_{j}^{*}(x)$ for all $i, j \in N$. Otherwise, there must exist $i, j \in N$ such that $\theta_{i}^{*}(x) \neq \theta_{j}^{*}(x)$. Without loss of generality, let $\theta_{i}^{*}(x)<$ $\theta_{j}^{*}(x)$. Define the $n$-tuple $\widehat{x}$ by

$$
\widehat{x}_{k}:= \begin{cases}x_{k}, & k \in N \backslash\{i, j\}, \\ x_{k}-\delta, & k=i, \\ x_{k}+\delta, & k=j .\end{cases}
$$


Here $\delta=\frac{\theta_{j}^{*}(x)-\theta_{j}^{*}(x)}{2}$. It is obvious that $\widehat{x} \in I^{*}(v)$. Then, the average compromised complaint vector of $\widehat{x}$ is

$$
\theta_{k}^{*}(\widehat{x})= \begin{cases}\theta_{k}^{*}(x), & k \in N \backslash\{i, j\}, \\ \theta_{k}^{*}(x)+\delta, & k=i, \\ \theta_{k}^{*}(x)-\delta, & k=j .\end{cases}
$$

Moreover, since $\delta>0$, we have

$$
\theta_{j}^{*}(x)>\theta_{j}^{*}(\widehat{x})=\theta_{i}^{*}(\widehat{x})>\theta_{i}^{*}(x) .
$$

This implies $\theta^{*}(\widehat{x})<_{L} \theta^{*}(x)$, which is contradiction with that $x$ lexicographically minimizes the average compromised complaint vector over the preimputation set. Hence, $\theta_{i}^{*}(x)=\theta_{j}^{*}(x)$ for all $i, j \in N$. By the efficiency of $x$ and Lemma 2.3, it is not difficult to obtain that $x_{i}=E N S C_{i}(v)$ for all $i \in N$.

We conclude this section with a simple example to illustrate the involved optimization models.

Example 2.2. Given a 3-person game $v \in \Gamma_{n}$ with $N=\{1,2,3\}$. Let the characteristic function $v$ be given by $v(1)=1, v(2)=2, v(3)=3, v(12)=4$, $v(23)=9, v(13)=8$ and $v(N)=10$. Then we have $b_{1}^{v}=1, b_{2}^{v}=2$ and $b_{3}^{v}=6$. It is easy to figure out that the ENSC value is $\operatorname{ENSC}(v)=\left(\frac{4}{3}, \frac{7}{3}, \frac{19}{3}\right)$. As to Problem 1, the following problem is taken into account

$$
\text { Minimize } \quad\left(x_{1}-1\right)^{2}+\left(x_{2}-2\right)^{2}+\left(x_{3}-6\right)^{2} \text {, s.t. } x_{1}+x_{2}+x_{3}=10 \text {. }
$$

The Lagrange function of this problem is

$$
L(x, \lambda)=\left(x_{1}-1\right)^{2}+\left(x_{2}-2\right)^{2}+\left(x_{3}-6\right)^{2}+\lambda\left(x_{1}+x_{2}+x_{3}-10\right),
$$

which gives the Lagrange conditions

$$
2\left(x_{1}-1\right)+\lambda=0 ; \quad 2\left(x_{2}-2\right)+\lambda=0 ; \quad 2\left(x_{3}-6\right)+\lambda=0 .
$$

Together with $x_{1}+x_{2}+x_{3}=10$, the optimal solution $x^{*}=\left(\frac{4}{3}, \frac{7}{3}, \frac{19}{3}\right)$ is exactly the ENSC value, verifying the validity of Theorem 2.1. 
As to Problem 2, we first denote the permutations on $N$ by $\pi_{1}=(1,2,3)$, $\pi_{2}=(1,3,2), \pi_{3}=(2,1,3), \pi_{4}=(2,3,1), \pi_{5}=(3,1,2)$ and $\pi_{6}=(3,2,1)$. The compromised ideal payoffs for the players are

$$
\begin{array}{ll}
\eta_{1}^{v \pi_{1}}=\eta_{1}^{v \pi_{2}}=2, & \eta_{1}^{v \pi_{3}}=\eta_{1}^{v \pi_{4}}=\eta_{1}^{v \pi_{5}}=\eta_{1}^{v \pi_{6}}=1, \\
\eta_{2}^{v \pi_{3}}=\eta_{2}^{v \pi_{4}}=3, & \eta_{2}^{v \pi_{1}}=\eta_{2}^{v \pi_{2}}=\eta_{2}^{v \pi_{5}}=\eta_{2}^{v \pi_{6}}=2, \\
\eta_{3}^{v \pi_{5}}=\eta_{3}^{v \pi_{6}}=7, & \eta_{3}^{v \pi_{1}}=\eta_{3}^{v \pi_{2}}=\eta_{3}^{v \pi_{3}}=\eta_{3}^{v \pi_{4}}=6 .
\end{array}
$$

Thus the explicit formulation of Problem 2 is

$$
\begin{array}{ll}
\text { Minimize } & 2\left(x_{1}-2\right)^{2}+4\left(x_{1}-1\right)^{2}+2\left(x_{2}-3\right)^{2} \\
& +4\left(x_{2}-2\right)^{2}+2\left(x_{3}-7\right)^{2}+4\left(x_{3}-6\right)^{2} \\
& \text { s.t. } \sum_{i=1}^{3} x_{i}=10 .
\end{array}
$$

The Lagrange function is

$$
\begin{gathered}
L(x, \lambda)=2\left(x_{1}-2\right)^{2}+4\left(x_{1}-1\right)^{2}+2\left(x_{2}-3\right)^{2}+4\left(x_{2}-2\right)^{2}+2\left(x_{3}-7\right)^{2}+ \\
4\left(x_{3}-6\right)^{2}+\lambda\left(x_{1}+x_{2}+x_{3}-10\right),
\end{gathered}
$$

which gives the Lagrange conditions

$$
\begin{aligned}
& 4\left(x_{1}-2\right)+8\left(x_{1}-1\right)+\lambda=0 \\
& 4\left(x_{2}-3\right)+8\left(x_{2}-2\right)+\lambda=0 ; \\
& 4\left(x_{3}-7\right)+8\left(x_{3}-6\right)+\lambda=0 .
\end{aligned}
$$

Together with $x_{1}+x_{2}+x_{3}=10$, the optimal solution $x^{*}=\left(\frac{4}{3}, \frac{7}{3}, \frac{19}{3}\right)$ coincides with the ENSC value, which verifies the validity of Theorem 2.2.

\subsection{Characterization of the ENSC value}

In Section 2.2, we implement the ENSC value as the unique optimal solution to some optimization problems. Alternative characterizations of the ENSC 
value will be shown in this section by proposing several new properties. Before doing that, we first list some usual principles that have been proposed to reflect the fairness and rationality for values. A value $\varphi: \Gamma_{n} \rightarrow \mathbf{R}^{n}$ satisfies

- Efficiency: if $\sum_{i \in N} \phi_{i}(v)=v(N)$ for all $v \in \Gamma_{n}$.

- Additivity: if $\varphi_{i}(v+w)=\varphi_{i}(v)+\varphi_{i}(w)$ for any $v, w \in \Gamma_{n}$.

- Invariance: if for any $a \in \mathbb{R}$ and $b \in \mathbb{R}^{n}$, it holds that $\varphi_{i}(a v+b)=$ $a \varphi_{i}(v)+b$, where $a v+b \in \Gamma_{n}$ is given by $(a v+b)(S):=a v(S)+\sum_{i \in S} b_{i}, \forall S \subseteq N$.

- Symmetry: if $\varphi_{i}(v)=\varphi_{j}(v)$, where $i, j \in N$ are symmetric players in game $v \in \Gamma_{n}$, that is, $v(S \cup i)=v(S \cup j), \forall S \subseteq N \backslash\{i, j\}$.

- Inessential game property: if $\varphi_{i}(v)=v(i), \forall i \in N$ for any inessential game $v \in \Gamma_{n}$, that is, $v(S)=\sum_{i \in S} v(i), \forall S \subseteq N$.

van den Brink [88] introduced the nullifying player property to characterize both the ED value and the CIS value. A player is nullifying if the worth of all coalitions containing him equals zero. The nullifying player property implies that the nullifying players will obtain nothing due to their nullifying influence to coalitions. Different from the nullifying players, we consider the players who bring nullifying influence to coalitions in an indirect way.

Definition 2.4. For any $v \in \Gamma_{n}$, player $i \in N$ is called a dual nullifying player in $v$ if $v(N)-v(N \backslash S)=0$ for all $S \subseteq N$ with $i \in S$.

Obviously, if a coalition $S$ contains a dual nullifying player, then the remaining part of the total worth $v(N)$ for it equals 0 whenever $v(N)$ is distributed in such a way that players outside $S$ receive the amount of $v(N \backslash S)$. In this case, an allocation rule should assign nothing to the dual nullifying players. This yields the dual nullifying player property. A value $\varphi: \Gamma_{n} \rightarrow \mathbf{R}^{n}$ satisfies

- Dual nullifying player property: if $\varphi_{i}(v)=0$, for any given $v \in \Gamma_{n}$ such that $i$ is a dual nullifying player in game $v$. 
By replacing the nullifying player property with dual nullifying player property in the characterization of the equal division value proposed by van den Brink [88], we obtain a new characterization of the equal division value.

Lemma 2.5. For all $v \in \Gamma_{n}$, the equal division value is the unique value that satisfies efficiency, additivity, symmetry and the dual nullifying player property.

Proof. Obviously, the equal division value satisfies efficiency, additivity and symmetry. Next we verify the dual nullifying player property for the equal division value. Suppose that player $i \in N$ is dual nullifying in game $v \in \Gamma_{n}$. By the definition of the dual nullifying player, we have $v(N)-v(N \backslash N)=$ $v(N)=0$, thus $E D_{i}(v)=\frac{v(N)}{n}=0$, which means that the dual nullifying property holds for the equal division value.

Uniqueness will be proved in a similar way as for the Shapley value but using the standard games instead of the unanimity games which are introduced by Harsanyi [37]. Now suppose that a value $\varphi: \Gamma_{n} \rightarrow \mathbb{R}^{n}$ satisfies these four properties. For any $T \varsubsetneqq N$, all players in $T$ are dual nullifying in game $b^{T}$. According to the dual nullifying player property, $\varphi_{i}\left(b^{T}\right)=0$ for all $i \in T$. By efficiency of $\varphi$, it holds that $\sum_{i \in N \backslash T} \varphi_{i}\left(b^{T}\right)=b^{T}(N)=0$. Moreover, all players in $N \backslash T$ are symmetric to each other, hence $\varphi_{i}\left(b^{T}\right)=0$ for all $i \in N \backslash T$.

For $T=N, \varphi_{i}\left(b^{N}\right)=\frac{b^{N}}{n}=\frac{1}{n}$ for all $i \in N$ simply by efficiency and symmetry.

Recall that the set $\left\{b^{T}\right\}_{T \subset N, T \neq \emptyset}$ forms a basis of $\Gamma_{n}$, yielding

$$
v=\sum_{T \subseteq N, T \neq \emptyset} v(T) b^{T} \text { for al } l v \in \Gamma_{n}
$$

Therefore additivity implies that $\varphi_{i}(v)=\sum_{T \subseteq N, T \neq \emptyset} v(T) \varphi_{i}\left(b^{T}\right)=\frac{v(N)}{n}$ for all $i \in N$, which completes the uniqueness part.

The ENSC value satisfies all the properties except the dual nullifying player property in Lemma 2.5 . This property holds for the ENSC value only on the domain of games of which the grand marginal contributions for all players equal 0. 
Definition 2.5. A game $v \in \Gamma_{n}$ is zero marginal normalized if $b_{i}^{v}=0$ for all $i \in N$.

A value $\varphi: \Gamma_{n} \rightarrow \mathbf{R}^{n}$ satisfies

- Zero marginal normalized game property: if $\varphi_{i}(v)=0$ for any zero marginal normalized game $v \in \Gamma_{n}$ in which $i$ is a dual nullifying player.

Clearly, the zero marginal normalized game property is generated by restricting the dual nullifying player property on zero marginal normalized games. Adding the invariance property and replacing the dual nullifying player property with the zero marginal normalized game property in Lemma 2.5 give a new characterization of the ENSC value.

Theorem 2.6. For all $v \in \Gamma_{n}$, the ENSC value is the unique value that satisfies efficiency, additivity, symmetry, invariance and the zero marginal normalized game property.

Proof. It is not difficult to verify that the ENSC value satisfies all above properties. It is sufficient to prove the uniqueness.

Let $\varphi: \Gamma_{n} \rightarrow \mathbf{R}^{n}$ be a value satisfying these five properties. For any $v \in \Gamma_{n}$, we define $w \in \Gamma_{n}$ as $w(S):=v(S)-\sum_{j \in S} b_{j}^{v}, \forall S \subseteq N$. It is obvious that $w$ is a zero marginal normalized game. Since $\varphi$ satisfies all properties for zero marginal normalized games in Lemma $2.5, \varphi$ coincides with the equal division value for such games. Thus $\varphi_{i}(w)=E D_{i}(v)=\frac{w(N)}{n}=\frac{v(N)-\sum_{j \in N} b_{j}^{v}}{n}$, $\forall i \in N$.

Moreover, $v=w+d$, where $d \in \mathbb{R}^{n}$ with $d_{i}=b_{i}^{v}$ for all $i \in N$. Together with invariance, it holds that $\varphi_{i}(v)=\varphi_{i}(w)+d_{i}=\frac{w(N)}{n}+b_{i}^{v}=\frac{v(N)-\sum_{j \in N} b_{j}^{v}}{n}+$ $b_{i}^{v}=E N S C_{i}(v)$ for all $i \in N$, which completes the uniqueness part.

A dual nullifying player not only neutralises productiveness of coalitions containing him, but also blocks cooperation within such coalitions. Discarding the neutralization effect gives a new kind of players called the dual dummifying players.

Definition 2.6. For any game $v \in \Gamma_{n}$, player $i \in N$ is a dual dummifying player in $v$ if $v(N)-v(N \backslash S)=\sum_{j \in S} b_{j}^{v}$ for all $S \subseteq N$ with $i \in S$. 
The remaining part of the total worth $v(N)$ for any coalition $S$ that contains a dual dummifying player is exactly the amount of all the grand marginal contributions of its members whenever coalition $N \backslash S$ receives the worth $v(N \backslash S)$. Analogously to the dual nullifying player property, we define the dummifying player property as follows. A value $\varphi: \Gamma_{n} \rightarrow \mathbf{R}^{n}$ satisfies:

- Dual dummifying player property: if $\varphi_{i}(v)=b_{i}^{v}$, for any $v \in \Gamma_{n}$ such that $i$ is a dual dummifying player in $v$.

As we showed in Section 2, the grand marginal contributions can be regarded as the ideal payoff for players which in general can not be guaranteed. While the dual dummifying player property requires this outcome for the dual dummifying player.

Theorem 2.7. For all $v \in \Gamma_{n}$, the ENSC value is the unique value that satisfies efficiency, additivity, symmetry and the dual dummifying player property.

Proof. It is easy to verify that the ENSC value satisfies efficiency, additivity and symmetry. We show that the ENSC value also has the dual dummifying player property. Let $i \in N$ be a dual dummifying player in game $v \in \Gamma_{n}$, then $v(N)=v(N)-v(N \backslash N)=\sum_{j \in N} b_{j}^{v}$. According to the definition of the ENSC value, we have $\operatorname{ENSC}_{i}(v)=b_{i}^{v}, \forall i \in N$, which completes the validity of the dual dummifying player property. Now it remains to prove the uniqueness.

Let $\varphi: \Gamma_{n} \rightarrow \mathbf{R}^{n}$ be a value that satisfies the mentioned properties. One may notice that the dual dummifying player property implies the dual nullifying player property restricted to zero marginal normalized games. In view of Theorem 2.6, it is sufficient to show that invariance holds. For any $v \in \Gamma_{n}$ and $b \in \mathbf{R}^{n}$, it remains to prove $\varphi_{i}(v+b)=\varphi_{i}(v)+b_{i}$, where $b(S):=\sum_{i \in S} b_{i}$, $\forall S \subseteq N$. By additivity, we have $\varphi(v+b)=\varphi(v)+\varphi(b)$. Moreover, since $b$ is an inessential game, all players are dual dummifying in $b$. The dual dummifying player property implies $\varphi_{i}(b)=b(N)-b(N \backslash i)=b_{i}, \forall i \in N$. Thus, the invariance property holds.

Monotonicity is a quite general standard for reasonable allocation in cooperative games. Young [102] characterized the Shapley value with strong monotonicity. The strong monotonicity states that if a game evolves such that some players' marginal contributions to all coalitions that contain them 
increase or stay unchanged then the payoff to these players will not decrease. Instead of discussing a situation which involves game changing, we introduce a new monotonicity named grand marginal contribution monotonicity which reveals the relation between payoffs to players in a given game. A value $\varphi: \Gamma_{n} \rightarrow \mathbf{R}^{n}$ satisfies

- Grand marginal contribution monotonicity: if $\varphi_{i}(v) \geq \varphi_{j}(v)$ for any $v \in \Gamma_{n}$ such that $b_{i}^{v} \geq b_{j}^{v}$, where $i, j \in N$.

Grand marginal contribution monotonicity expresses the fact that players with larger grand marginal contribution will be assigned with a larger portion of the total benefit. Together with efficiency, additivity and inessential game property, we derive a new characterization of the ENSC value.

Theorem 2.8. For all $v \in \Gamma_{n}$, the ENSC value is the unique value that satisfies efficiency, additivity, inessential game property and grand marginal contribution monotonicity.

Proof. It is clear that the ENSC value satisfies the properties mentioned in the theorem. It remains to prove the uniqueness part.

Given a value $\varphi: \Gamma_{n} \rightarrow \mathbf{R}^{n}$ that satisfies the four properties. For any $v \in \Gamma_{n}$, we decompose $v$ into two games, i.e., $v=u+w$, where $u(S):=v(S)-\sum_{j \in S} b_{j}^{v}$ and $w(S):=\sum_{j \in S} b_{j}^{v}, \forall S \subseteq N$. Obviously, $w$ is an inessential game, thus $\varphi_{i}(w)=w(i)=v(N)-v(N \backslash i)=b_{i}^{v}$ by applying inessential game property of $\varphi$.

For any $i \in N, b_{i}^{u}=u(N)-u(N \backslash i)=\sum_{k \in N} b_{k}^{v}$, which indicates that all players have the same marginal contribution to the grand coalition in game $u$. According to grand marginal contribution monotonicity of $\varphi$, we have $\varphi_{i}(u)=\frac{u(N)}{n}=\frac{v(N)-\sum_{j \in N} b_{j}^{v}}{n}, \forall i \in N$. Finally, additivity implies that $\varphi_{i}(v)=$ $\varphi_{i}(u+w)=\varphi_{i}(u)+\varphi_{i}(w)=\frac{v(N)-\sum_{j \in N} b_{j}^{v}}{n}+b_{i}^{v}=\operatorname{ENSC}_{i}(v)$.

Remark 2.2. To show the independence of the four axioms in Theorem 2.8, note:

1. The value $\varphi_{i}^{1}(v):=v(N)-v(N \backslash i)$ for all $i \in N$ satisfies linearity, inessential game property and grand marginal contribution monotonicity. But it does not satisfy efficiency. 
2. The value $\varphi_{i}^{2}(v):=\frac{v(N)-v(N \backslash i)}{\sum_{j \in N}(v(N)-v(N \backslash j))} v(N)$ for all $i \in N$ satisfies efficiency, inessential game property and grand marginal contribution monotonicity. But it does not satisfy linearity.

3. The value $\varphi_{i}^{3}(v):=\frac{v(N)}{n}$ for all $i \in N$ satisfies efficiency, linearity and grand marginal contribution monotonicity. But it does not satisfy inessential game property.

4. The value $\varphi_{i}^{4}(v):=v(i)+\frac{v(N)-\sum_{j \in N} v(j)}{n}$ for all $i \in N$ satisfies efficiency, linearity and inessential game property. But it does not satisfy grand marginal contribution monotonicity. 



\section{Chapter 3}

\section{Implementation of the $\alpha$-ENSC value}

In this chapter, we introduce a new solution concept called $\alpha$-ENSC value which is a convex combination of the ENSC value and the ED value. The $\alpha$-ENSC value reconciles two economic thoughts: marginalism and egalitarianism. We study an allocation process under the assumption that players are partially egocentric, and the final outcome happens to be the $\alpha$-ENSC value. The $\alpha$-ENSC value is also the solution for corresponding optimization models under certain complaint criterion. Several new properties are proposed to characterize the $\alpha$-ENSC value, including $\alpha$-dual individual rationality, $\alpha$ egocentric inessential game property and grand marginal contribution monotonicity.

\subsection{Introduction}

In 1953, Shapley [72] introduced the Shapley value, which assigns to each player a payoff measuring his productivity within a cooperative game. Driessen [23] interpreted the Shapley value as an average of marginal contributions, i.e., players enter the game one by one in the order $(\pi(1), \pi(2), \cdots, \pi(n))$, where $\pi$ is a bijection on the player set, and each player obtains the marginal 
contribution he creates by joining the group of players already present, and the Shapley value averages all marginal vectors. Since every new entrant obtains his marginal contribution and does not share it with other players, in this sense, the Shapley value can be seen as an egoistic or self-interested allocation rule.

However, the grand marginal contributions may not be the ideal payoff of the players. Gillies [29] stated that the grand marginal contribution is an upper bound of the core, which is the set of feasible allocations that cannot be improved upon by any coalition. When considering an allocation in the core, the closer to the grand marginal contribution, the happier the player is. Under this circumstance, all the players would like to obtain their grand marginal contributions and do not have incentive to share it with other players. As discussed in Chapter 2, the ENSC value is derived based on the egotism of players. In contrast, we consider the so-called altruistic players. Recall that a null player gets $\frac{v(N)}{n}$ under the ED value although his marginal contributions are zero. All the non-null players are in some sense altruistic. Therefore, the ED value can be understood as an allocation under the principle of "altruism".

In this chapter, we consider the convex combination of the ED value and the the ENSC value, namely the $\alpha$-ENSC value.

Definition 3.1. For any game $(N, v) \in \Gamma_{n}$ and $\alpha \in[0,1]$, the $\alpha$-ENSC value is defined as

$$
\alpha-E N S C_{i}(v)=\alpha E N S C_{i}(v)+(1-\alpha) E D_{i}(v), \quad \text { for all } \quad i \in N .
$$

We can rewrite the formula of the $\alpha$-ENSC value as

$$
\alpha-E N S C_{i}(v)=\alpha b_{i}^{v}+\frac{1}{n}\left[v(N)-\sum_{j \in N} \alpha b_{j}^{v}\right], \quad \text { for all } i \in N .
$$

In view of Equation (3.2), the $\alpha$-ENSC value can also be regarded as a generalization of the ENSC value by taking $0 \leq b_{i}^{v} \leq v(N)-v(N \backslash i)$ in Definition 3.1. The parameter $\alpha$ reflects the degree of egotism for players towards their grand marginal contribution. 
This value reconciles two thoughts: egocentrism and altruism. By introducing an individual preference parameter $\alpha$, where $\alpha \in[0,1]$, it reflects the players' predilection to the grand marginal contribution. We study the dynamic preference situation which has the altruistic behavior and egocentric behavior as two extreme cases. When $\alpha=0$, the player is totally altruistic, and the player is totally egocentric while $\alpha=1$. The larger $\alpha$ is, the more selfish the player is. In order to reflect these two attitudes, several other combinatorial values have also been proposed. Notice that the $\alpha$-ENSC value belongs to the class of the equal surplus sharing solutions, which were introduced by van den Brink and Funaki [89]. We propose a process implementation of the value and study the value as the solution of some optimization models. van den Brink and Funaki [90] characterized the convex combination of the Shapley value and the ED value, namely the $\alpha$ egalitarian Shapley value introduced by Joosten et al. $[40,41]$. When compared to the ENSC value, the Shapley value seems less egocentric as an extreme point. Xu et al. [97] introduced the $\alpha$-CIS value as the convex combination of the CIS value and the ED value. But what they took into account is just the minimal right for players, that is, players' individual worths. For that reason, the $\alpha$-CIS value may fail to embody the egocentric part properly.

Moreover, we introduce a new kind of complaints for individual players instead of considering coalitions. In reality, players may be neither totally egocentric nor altruistic, and it is possible that the new entrant only obtains part of his grand marginal contribution. For different grand coalition forming orders, we assumed that all players are partially egocentric as we have announced, which means players could only get a fraction of his grand marginal contribution and the remaining money or utilities shared equally among the players who have come before him. By considering players' preference to the grand marginal contribution, it corresponds to the ideal payoff vector of individual players, yielding a gap between ideal payoff and proposed allocation, the difference of which is called the complaint.

Our aim is to minimize the total variance of complaint for players among all formation orders under the least square criterion, which is similar to the method as Ruiz et al. [68-70] have applied. Interestingly, the unique optimal solution for this problem coincides with the $\alpha$-ENSC value. Moreover, the 
expected ideal payoff, which is the average ideal payoff deprived from considering all formation orders, also coincides with the $\alpha$-ENSC value. One may notice that this process seems similar to the "procedural" value proposed by Malawski [47]. What players share during the process is the grand marginal contributions rather than marginal contributions to the coalitions formed by players joining in random order. Besides, the $\alpha$-ENSC value does not belong to the class of the procedural values, since it fails to satisfy weakly monotonic, which is one of the axiomatizations to characterize a "procedural" value.

van den Brink and Funaki [89] characterized the ENSC value by means of dual individual rationality, which is under the assumption that all players regard their grand marginal contribution as ideal payoff. Notice that players in our model are partially egocentric, we propose a new property called $\alpha$ dual individual rationality. Interestingly, together with efficiency, linearity and symmetry, $\alpha$-dual individual rationality characterizes the $\alpha$-ENSC value. Moreover, some other new properties are also proposed to characterize the $\alpha$ ENSC value, including the $\alpha$-egocentric inessential game property and grand marginal contribution monotonicity. All of them reflect the dual characters of players: egocentrism and altruism.

\subsection{Procedural implementation of the $\alpha$-ENSC value}

Given a formation order, a player who claims all his contribution to the grand coalition when he joins the game is totally egocentric. But the general case is that players may also possess the altruistic quality and be partially egocentric. We will incarnate the degree of egocentrism by a parameter. The larger this parameter is, the more egocentric players are. A player is partially egocentric, if for any coalition formation order in which he is not the first entrant, he would like to obtain a proportion of his grand marginal contribution when he joins the game and what's left is distributed equally among the players who have come before him. Formally, we give the definition of partially egoistic player as in Definition 2.1.

Definition 3.2. For any $v \in \Gamma_{n}, \alpha \in[0,1]$ and $\pi \in \Pi(N)$, where $\Pi(N)$ denotes the set of all permutations on $N$, a player $i \in N$ is $\alpha$ egoistic if he claims $C_{i}^{\alpha \pi}$ 
when he joins the game, where

$$
C_{i}^{\alpha \pi}:= \begin{cases}v(i), & \text { if } \pi(i)=1, \\ \alpha b_{i}^{v}, & \text { otherwise. }\end{cases}
$$

The gap $v\left(S_{\pi}^{i}\right)-v\left(S_{\pi}^{i} \backslash i\right)-C_{i}^{\alpha \pi}$ generated by player $i$ is shared among his predecessors. The portion for player $k \in N$ is $G_{i k}^{\pi}$, i.e.,

$$
G_{i k}^{\alpha \pi}:= \begin{cases}\frac{v\left(S_{\pi}^{i}\right)-v\left(S_{\pi}^{i} \backslash i\right)-C_{i}^{\alpha \pi}}{\pi(i)-1}, & \text { if } k \in S_{\pi}^{i} \backslash\{i\}, \\ 0, & \text { otherwise. }\end{cases}
$$

Here $S_{\pi}^{i}:=\{j \in N: \pi(j) \leq \pi(i)\}$ denotes the set that consists of player $i$ and all his predecessors under permutation $\pi$ and $\pi(i)$ is the position of player $i$ in order $\pi$.

To be special, when $\alpha=0$, the new comer will obtain 0 . If $\alpha=1$, the new comer takes all his grand marginal contribution. We illustrate this thought by considering a three-person game.

Example 3.1. Given that all players are $\alpha$ egoistic, and the entrance order is $\pi=(3,1,2)$.

At the beginning, player 3 joins the game. Since he is the first entrant, the payoff is his individual worth $v(3)$, while player 1 and 2 get nothing. Payoff allocation for players at the moment is shown in Table 1.

TABLE 3.1 Payoff allocation when player 3 joins in.

\begin{tabular}{lccc}
\hline Player & $\mathbf{1}$ & $\mathbf{2}$ & $\mathbf{3}$ \\
\hline Allocation & 0 & 0 & $v(3)$ \\
\hline
\end{tabular}

Then, player 1 arrives. As we have assumed that players are all $\alpha$ partially egoistic, player 1 gets $\alpha$ times his grand marginal contribution. The remaining worth $v(1,3)-v(3)-\alpha b_{1}^{v}$ is charged by player 3 , while player 2 still obtains nothing. Table 2 shows the payoff allocation to players in this step.

Finally, the last player 2 joins and he obtains $\alpha$ times his grand marginal contribution and what's left is shared among players 1,3 equally. Table 3 shows the outcome. 
TABLE 3.2 Payoff allocation when player 1 joins in.

\begin{tabular}{lccc}
\hline Player & $\mathbf{1}$ & $\mathbf{2}$ & $\mathbf{3}$ \\
\hline Allocation & $\alpha b_{1}^{v}$ & 0 & $v(1,3)-v(3)-\alpha b_{1}^{v}$ \\
\hline
\end{tabular}

TABLE 3.3 Payoff allocation when player 2 joins in.

\begin{tabular}{lccc}
\hline Player & $\mathbf{1}$ & $\mathbf{2}$ & $\mathbf{3}$ \\
\hline Allocation & $\frac{v(N)-v(1,3)-\alpha b_{2}^{v}}{2}$ & $\alpha b_{2}^{v}$ & $\frac{v(N)-v(1,3)-\alpha b_{2}^{v}}{2}$ \\
\hline
\end{tabular}

Summate the payoffs in different three stages, then we obtain the total payoff to players as is showed in Table 4.

TABLE 3.4 Payoff for $\alpha$ partially egoistic players under formation procedure $\pi=(3,1,2)$

\begin{tabular}{lcccc}
\hline Player $\backslash$ Step & $\mathbf{1}$ & $\mathbf{2}$ & $\mathbf{3}$ & Player's final payoff \\
\hline $\mathbf{3}$ & $v(3)$ & $v(1,3)-v(3)-\alpha b_{1}^{v}$ & $\frac{v(N)-v(1,3)-\alpha b_{2}^{v}}{2}$ & $-\alpha b_{1}^{v}+\frac{v(N)+v(1,3)-\alpha b_{2}^{v}}{2}$ \\
$\mathbf{1}$ & 0 & $\alpha b_{1}^{v}$ & $\frac{v(N)-v(1,3)-\alpha b_{2}^{v}}{2}$ & $\alpha b_{1}^{v}+\frac{v(N)-v(1,3)-\alpha b_{2}^{v}}{2}$ \\
$\mathbf{2}$ & 0 & 0 & $\alpha b_{2}^{v}$ & $\alpha b_{2}^{v}$ \\
\hline
\end{tabular}

Obviously, the final allocation scheme satisfies efficiency. As mentioned in Chapter 2, we call the final payoff in such procedure the partially compromised ideal payoff because under the assumption that all players are $\alpha$ egoistic, every player in any coalition formation order gets what he wants but also has to undertake the gap generated by his successors. Now we give the general explicit expression of the total payoff to players in this procedure as follows:

Definition 3.3. Given that all players are $\alpha$ egoistic and for the coalition formation order $\pi$, player $i^{\prime} s$ partially compromised ideal payoff is 


$$
\eta_{i}^{\alpha \pi}= \begin{cases}v(i)+\sum_{k=\pi(i)+1}^{n} \frac{v\left(S_{\pi}^{\pi^{-1}(k)}\right)-v\left(S_{\pi}^{\pi^{-1}(k)} \backslash \pi^{-1}(k)\right)-\alpha b_{\pi^{-1}(k)}^{v}}{k-1}, & \pi(i)=1, \\ \alpha b_{i}^{v}+\sum_{k=\pi(i)+1}^{n} \frac{v\left(S_{\pi}^{\pi^{-1}(k)}\right)-v\left(S_{\pi}^{\pi^{-1}(k)} \backslash \pi^{-1}(k)\right)-\alpha b_{\pi^{-1}(k)}^{v}}{k-1}, & \pi(i) \neq 1, n, \\ \alpha b_{i}^{v}, & \pi(i)=n .\end{cases}
$$

The next theorem reveals the fact that the ultimate payoff is identical to the $\alpha$-ENSC value when all formation orders are taken into consideration.

Theorem 3.1. For any $(N, v) \in \Gamma_{n}$, given that all the players are $\alpha$ egocentric, then player i's expected ideal payoff over all permutations coincides with his $\alpha$-ENSC value, i.e.,

$$
\sum_{\pi \in \Pi(N)} \frac{1}{n !} \eta_{i}^{\alpha \pi}=\alpha-E N S C_{i}(v)=\alpha E N S C_{i}(v)+(1-\alpha) E D_{i}(v),
$$

where $\eta_{i}^{\alpha \pi}$ has the form in Equation (3.5).

In order to prove the Theorem, the following lemma is taken into account.

Lemma 3.2. For any cooperative game $(N, v) \in \Gamma_{n}$, the $E D$ value has the form as follows:

$$
E D_{i}(v)=\sum_{\pi: \pi(i)=1} \frac{1}{n !}\left[v(i)+\sum_{k=\pi(i)+1}^{n} \frac{m_{\pi(k)}}{k-1}\right]+\sum_{\pi: \pi(i) \neq 1, n} \frac{1}{n !} \sum_{k=\pi(i)+1}^{n} \frac{m_{\pi(k)}}{k-1},
$$

where $m_{\pi(k)}=v\left(S_{\pi}^{\pi^{-1}(k)}\right)-v\left(S_{\pi}^{\pi^{-1}(k)} \backslash \pi^{-1}(k)\right)$.

Proof.

$$
\begin{aligned}
& \sum_{\pi: \pi(i)=1} \frac{1}{n !}\left[v(i)+\sum_{k=\pi(i)+1}^{n} \frac{m_{\pi(k)}}{k-1}\right]+\sum_{\pi: \pi(i) \neq 1, n} \frac{1}{n !} \sum_{k=\pi(i)+1}^{n} \frac{m_{\pi(k)}}{k-1} \\
= & \frac{(n-1) !}{n !} v(i)+\sum_{\pi: \pi(i)=1} \frac{1}{n !} \sum_{k=\pi(i)+1}^{n} \frac{m_{\pi(k)}}{k-1}+\sum_{\pi: \pi(i) \neq 1, n} \frac{1}{n !} \sum_{k=\pi(i)+1}^{n} \frac{m_{\pi(k)}}{k-1}
\end{aligned}
$$




$$
\begin{aligned}
& =\frac{1}{n} v(i)+\sum_{\pi(i) \neq n} \frac{1}{n !} \sum_{k=\pi(i)+1}^{n} \frac{m_{\pi(k)}}{k-1} \\
& =\frac{1}{n} v(i)+\sum_{l \in N \backslash\{i\}} \sum_{\pi: S_{\pi}^{l} \ni i} \frac{1}{n !} \frac{v\left(S_{\pi}^{l}\right)-v\left(S_{\pi}^{l} \backslash l\right)}{\left|S_{\pi}^{l}\right|-1} \\
& =\frac{1}{n} v(i)+\sum_{l \in N \backslash\{i\}} \sum_{S \ni i, l} \frac{v(S)-v(S \backslash l)}{s-1} \cdot \frac{(s-1) !(n-s) !}{n !} \\
& =\frac{1}{n} v(i)+\sum_{l \in N \backslash\{i\}} \sum_{S \ni i, l}[v(S)-v(S \backslash l)] \cdot \frac{(s-2) !(n-s) !}{n !} \\
& =\frac{1}{n} v(i)+\sum_{l \in N \backslash\{i\}} \sum_{S \ni i, l} v(S) \cdot \frac{(s-2) !(n-s) !}{n !}-\sum_{l \in N \backslash\{i\}} \sum_{S \ni i, l} v(S \backslash l) \cdot \frac{(s-2) !(n-s) !}{n !} \\
& =\frac{1}{n} v(i)+\sum_{S \ni i,|S| \geq 2} \sum_{l \in S \backslash\{i\}} v(S) \cdot \frac{(s-2) !(n-s) !}{n !} \\
& -\sum_{l \in N \backslash\{i\}} \sum_{T \subseteq N \backslash l, T \ni i} v(T) \cdot \frac{(t+1-2) !(n-(t+1)) !}{n !} \\
& =\frac{1}{n} v(i)+\sum_{S \ni i,|S| \geq 2}(s-1) v(S) \cdot \frac{(s-2) !(n-s) !}{n !}-\sum_{T \ni i,|T| \leq n-1} \sum_{l \notin T} v(T) \cdot \frac{(t-1) !(n-t-1) !}{n !} \\
& =\frac{1}{n} v(i)+\sum_{S \ni i,|S| \geq 2} v(S) \cdot \frac{(s-1) !(n-s) !}{n !}-\sum_{T \ni i,|T| \leq n-1}(n-t) v(T) \cdot \frac{(t-1) !(n-t-1) !}{n !} \\
& =\frac{1}{n} v(i)+\sum_{S \ni i,|S| \geq 2} v(S) \cdot \frac{(s-1) !(n-s) !}{n !}-\sum_{T \ni i,|T| \leq n-1} v(T) \cdot \frac{(t-1) !(n-t) !}{n !} \\
& =\frac{1}{n} v(i)-\frac{1}{n} v(i)+\frac{v(N)}{n} \\
& =\frac{v(N)}{n}=E D_{i}(v)
\end{aligned}
$$

Together with Lemma 2.3, we now provide the proof of Theorem 3.1. Proof of Theorem 3.1. In view of Equation (3.5), we have

$$
\sum_{\pi \in \Pi(N)} \frac{1}{n !} \eta_{i}^{\alpha \pi}=\sum_{\pi: \pi(i)=1} \frac{1}{n !} \eta_{i}^{\alpha \pi}+\sum_{\pi: \pi(i) \neq 1, n} \frac{1}{n !} \eta_{i}^{\alpha \pi}+\sum_{\pi: \pi(i)=n} \frac{1}{n !} \eta_{i}^{\alpha \pi}
$$




$$
\begin{aligned}
& =\sum_{\pi: \pi(i)=1} \frac{1}{n !}\left[v(i)+\sum_{k=\pi(i)+1}^{n} \frac{\left.m_{\pi(k)}-\alpha b_{\pi^{-1}(k)}^{v}\right]}{k-1}\right. \\
& +\sum_{\pi: \pi(i) \neq 1, n} \frac{1}{n !}\left[\alpha b_{i}^{v}+\sum_{k=\pi(i)+1}^{n} \frac{\left.m_{\pi(k)}-\alpha b_{\pi^{-1}(k)}^{v}\right]+\frac{(n-1) !}{n !} \alpha b_{i}^{v}}{k-1}\right. \\
& =\sum_{\pi: \pi(i)=1} \frac{1}{n !}\left[\alpha v(i)+(1-\alpha) v(i)+\sum_{k=\pi(i)+1}^{n} \frac{\alpha\left(m_{\pi(k)}-b_{\pi^{-1}(k)}^{v}\right)+(1-\alpha) m_{\pi(k)}}{k-1}\right] \\
& +\sum_{\pi: \pi(i) \neq 1, n} \frac{1}{n !}\left[\alpha b_{i}^{v}+\sum_{k=\pi(i)+1}^{n} \frac{\left.\alpha m_{\pi(k)}-\alpha b_{\pi^{-1}(k)}+\frac{(1-\alpha) m_{\pi(k)}}{k-1}\right]+\frac{1}{n} \alpha b_{i}^{v}}{k-1}\right. \\
& =\frac{1}{n} \alpha b_{i}^{v}+\sum_{\pi: \pi(i)=1} \frac{1}{n !}\left[\alpha v(i)+\sum_{k=\pi(i)+1}^{n} \frac{\left.\alpha m_{\pi(k)}-\alpha b_{\pi^{-1}(k)}^{v}\right]}{k-1}\right. \\
& +\sum_{\pi: \pi(i) \neq 1, n} \frac{1}{n !}\left[\alpha b_{i}^{v}+\sum_{k=\pi(i)+1}^{n} \frac{\left.\alpha m_{\pi(k)}-\alpha b_{\pi^{-1}(k)}^{v}\right]}{k-1}\right. \\
& +\sum_{\pi: \pi(i)=1} \frac{1}{n !}\left[(1-\alpha) v(i)+\sum_{k=\pi(i)+1}^{n} \frac{\left.(1-\alpha) m_{\pi(k)}\right]}{k-1}\right] \\
& +\sum_{\pi: \pi(i) \neq 1, n} \frac{1}{n !} \sum_{k=\pi(i)+1}^{n} \frac{(1-\alpha) m_{\pi(k)}}{k-1} \\
& =\alpha E N S C_{i}(v)+(1-\alpha) E D_{i}(v) \\
& =\alpha-E N S C_{i}(v)
\end{aligned}
$$

The last but one equation is derived from Lemma 2.3 and Lemma 3.2.

Malawski [47] introduced the concept of "procedural" values, the family of which includes several classical values for cooperative games, such as the Shapley value, the ED value and solidarity value. Although the $\alpha$-ENSC value also has the procedural form, it does not belong to the family since it fails to obey the weakly monotonic property proposed by Weber [96], which is crucial in one of the axiomatizations to characterize the "procedural"values. 


\subsection{The general $\alpha$-ENSC value}

We now consider a less restrictive situation, in which players have different degrees of altruism and egoism in the model. A more general result will be derived under such an assumption. Let $\alpha_{i}$ denote the degree of egoism for player $i$. Similar to the procedure in Example 3.1, players join the game one by one, but every new entrant $i$ obtains $\alpha_{i}$ times his grand marginal contribution, then the corresponding ideal payoff with respect to coalition formation order $\pi$ is as follows.

$$
\eta_{i}^{\vec{\alpha} \pi}= \begin{cases}v(i)+\sum_{k=\pi(i)+1}^{n} \frac{v\left(S_{\pi}^{\pi^{-1}(k)}\right)-v\left(S_{\pi}^{\pi^{-1}(k)} \backslash \pi^{-1}(k)\right)-\alpha_{\pi^{-1}(k)} b_{\pi^{-1}(k)}^{v}}{k-1}, & \pi(i)=1, \\ \alpha_{i} b_{i}^{v}+\sum_{k=\pi(i)+1}^{n} \frac{v\left(S_{\pi}^{\pi^{-1}(k)}\right)-v\left(S_{\pi}^{\pi^{-1}(k)} \backslash \pi^{-1}(k)\right)-\alpha_{\pi^{-1}(k)} b_{\pi^{-1}(k)}^{v}}{k-1}, & \pi(i) \neq 1, n, \\ \alpha_{i} b_{i}^{v}, & \pi(i)=n .\end{cases}
$$

Without loss of generality, we write $\alpha_{1} \leq \alpha_{2} \leq \cdots \leq \alpha_{n}$. For any $i \in N$, when considering all the formation orders of the grand coalition, the average final payoff is given by

$$
\sum_{\pi \in \Pi(N)} \frac{1}{n !} \eta_{i}^{\vec{\alpha} \pi}=\alpha_{1} \operatorname{ENSC}_{i}(v)+\sum_{k=2}^{n}\left(\alpha_{k}-\alpha_{k-1}\right) E N S C_{i}\left(v^{k}\right)+\left(1-\alpha_{n}\right) E D_{i}(v),
$$

where $v^{k}, k \in\{2,3, \cdots, n\}$, is defined as

$$
v^{k}(S)= \begin{cases}v(N), & \text { if } S=N \backslash\{l\}, l<k, \\ v(S), & \text { otherwise }\end{cases}
$$

The proof of Equation (3.8) entails the decomposition of the payoff in every step for players. Notice that the degree of egoism $\alpha_{i}$ for player $i$ is decomposed into different levels, $\alpha_{1}, \alpha_{2}-\alpha_{1}, \cdots, \alpha_{i}-\alpha_{i-1}$. For games $v^{k}$ with $k \leq i$, player $i$ will obtain a portion of the ENSC value as his egoistic action. Once $k>i$, the grand marginal contribution for $i$ in game $v^{k}$ equals zero and what he gets in these games is just originated from other players' altruistic action. Moreover, the $\alpha$-ENSC value is one of the special cases when all the degrees are the same. 
Proof of Equation (3.8).

$$
\begin{aligned}
& \sum_{\pi \in \Pi(N)} \frac{1}{n !} \eta_{i}^{\vec{\alpha} \pi}=\sum_{\pi: \pi(i)=n} \frac{1}{n !} \eta_{i}^{\vec{\alpha} \pi}+\sum_{\pi: \pi(i)=1} \frac{1}{n !} \eta_{i}^{\vec{\alpha} \pi}+\sum_{\pi: \pi(i) \neq 1, n} \frac{1}{n !} \eta_{i}^{\vec{\alpha} \pi} \\
& =\frac{1}{n} \alpha_{i} b_{i}^{v}+\sum_{\pi: \pi(i)=1} \frac{1}{n !}\left[v(i)+\sum_{k=\pi(i)+1}^{n} \frac{m_{\pi(k)}-\alpha_{\pi^{-1}(k)} b_{\pi^{-1}(k)}^{v}}{k-1}\right] \\
& +\sum_{\pi: \pi(i) \neq 1, n} \frac{1}{n !}\left[\alpha_{i} b_{i}^{v}+\sum_{k=\pi(i)+1}^{n} \frac{m_{\pi(k)}-\alpha_{\pi^{-1}(k)} b_{\pi^{-1}(k)}^{v}}{k-1}\right]+\frac{(n-1) !}{n !} \alpha_{i} b_{i}^{v} \\
& =\frac{1}{n}\left[\alpha_{1} b_{i}^{v}+\sum_{j=2}^{i}\left(\alpha_{j}-\alpha_{j-1}\right) b_{i}^{v}\right] \\
& +\sum_{\pi: \pi(i)=1} \frac{1}{n !}\left[\alpha_{1} v(i)+\sum_{j=2}^{n}\left(\alpha_{j}-\alpha_{j-1}\right) v(i)+\left(1-\alpha_{n}\right) v(i)\right] \\
& +\sum_{\pi: \pi(i)=1} \frac{1}{n !} \sum_{k=\pi(i)+1}^{n} \frac{1}{k-1}\left[\alpha_{1} m_{\pi(k)}+\sum_{j=2}^{n}\left(\alpha_{j}-\alpha_{j-1}\right) m_{\pi(k)}+\left(1-\alpha_{n}\right) m_{\pi(k)}\right] \\
& \text { - } \sum_{\pi: \pi(i)=1} \frac{1}{n !} \sum_{k=\pi(i)+1}^{n} \frac{1}{k-1}\left[\alpha_{1} b_{\pi^{-1}(k)}^{v}+\sum_{j=2}^{\pi^{-1}(k)}\left(\alpha_{j}-\alpha_{j-1}\right) b_{\pi^{-1}(k)}^{v}\right] \\
& +\sum_{\pi: \pi(i) \neq 1, n} \frac{1}{n !}\left[\alpha_{1} b_{i}^{v}+\sum_{j=2}^{i}\left(\alpha_{j}-\alpha_{j-1}\right) b_{i}^{v}\right] \\
& +\sum_{\pi: \pi(i) \neq 1, n} \frac{1}{n !} \sum_{k=\pi(i)+1}^{n} \frac{1}{k-1}\left[\alpha_{1} m_{\pi(k)}+\sum_{j=2}^{n}\left(\alpha_{j}-\alpha_{j-1}\right) m_{\pi(k)}+\left(1-\alpha_{n}\right) m_{\pi(k)}\right] \\
& \text { - } \sum_{\pi: \pi(i) \neq 1, n} \frac{1}{n !} \sum_{k=\pi(i)+1}^{n} \frac{1}{k-1}\left[\alpha_{1} b_{\pi^{-1}(k)}^{v}+\sum_{j=2}^{\pi^{-1}(k)}\left(\alpha_{j}-\alpha_{j-1}\right) b_{\pi^{-1}(k)}^{v}\right] \\
& =\frac{1}{n}\left[\alpha_{1} b_{i}^{v}+\sum_{j=2}^{n}\left(\alpha_{j}-\alpha_{j-1}\right) b_{i}^{v^{j}}\right] \\
& +\sum_{\pi: \pi(i)=1} \frac{1}{n !}\left[\alpha_{1} v(i)+\sum_{j=2}^{n}\left(\alpha_{j}-\alpha_{j-1}\right) v(i)+\left(1-\alpha_{n}\right) v(i)\right] \\
& +\sum_{\pi: \pi(i)=1} \frac{1}{n !} \sum_{k=\pi(i)+1}^{n} \frac{1}{k-1}\left[\alpha_{1} m_{\pi(k)}+\sum_{j=2}^{n}\left(\alpha_{j}-\alpha_{j-1}\right) m_{\pi(k)}+\left(1-\alpha_{n}\right) m_{\pi(k)}\right]
\end{aligned}
$$




$$
\begin{aligned}
& -\sum_{\pi: \pi(i)=1} \frac{1}{n !} \sum_{k=\pi(i)+1}^{n} \frac{1}{k-1}\left[\alpha_{1} b_{\pi^{-1}(k)}^{v}+\sum_{j=2}^{n}\left(\alpha_{j}-\alpha_{j-1}\right) b_{\pi^{-1}(k)}^{v^{j}}\right] \\
& +\sum_{\pi: \pi(i) \neq 1, n} \frac{1}{n !}\left[\alpha_{1} b_{i}^{v}+\sum_{j=2}^{n}\left(\alpha_{j}-\alpha_{j-1}\right) b_{i}^{v^{j}}\right] \\
& +\sum_{\pi: \pi(i) \neq 1, n} \frac{1}{n !} \sum_{k=\pi(i)+1}^{n} \frac{1}{k-1}\left[\alpha_{1} m_{\pi(k)}+\sum_{j=2}^{n}\left(\alpha_{j}-\alpha_{j-1}\right) m_{\pi(k)}\right] \\
& -\sum_{\pi: \pi(i) \neq 1, n} \frac{1}{n !} \sum_{k=\pi(i)+1}^{n} \frac{1}{k-1}\left[\alpha_{1} b_{\pi^{-1}(k)}^{v}+\sum_{j=2}^{n}\left(\alpha_{j}-\alpha_{j-1}\right) b_{\pi^{-1}(k)}^{v^{j}}\right] \\
& =\alpha_{1} E N S C_{i}(v)+\sum_{k=2}^{n}\left(\alpha_{k}-\alpha_{k-1}\right) E N S C_{i}\left(v^{k}\right)+\left(1-\alpha_{n}\right) E D_{i}(v) .
\end{aligned}
$$

The last equation is derived from Lemma 2.3 and Lemma 3.2.

We conclude this section by reconsidering Example 3.1 to illustrate the validity of Equation (3.8).

Example 3.2. For any $v \in \Gamma_{n}$, we suppose that player $i$ is $\alpha_{i}$ egoistic and the entrance order is $\pi=(3,1,2)$. Here we omit the allocation for players in every step and only show the final payoff under this entrance order in Table 3.5 .

TABLE 3.5 Payoff for players under formation procedure $\pi=$ $(3,1,2)$

\begin{tabular}{lcccc}
\hline Player $\backslash$ Step & $\mathbf{1}$ & $\mathbf{2}$ & $\mathbf{3}$ & Player's final payoff \\
\hline $\mathbf{3}$ & $v(3)$ & $v(1,3)-v(3)-\alpha_{1} b_{1}^{v}$ & $\frac{v(N)-v(1,3)-\alpha_{2} b_{2}^{v}}{2}$ & $-\alpha_{1} b_{1}^{v}+\frac{v(N)+v(1,3)-\alpha_{2} b_{2}^{v}}{2}$ \\
$\mathbf{1}$ & 0 & $\alpha_{1} b_{1}^{v}$ & $\frac{v(N)-v(1,3)-\alpha_{2} b_{2}^{v}}{2}$ & $\alpha_{1} b_{1}^{v}+\frac{v(N)-v(1,3)-\alpha b_{2}^{v}}{\alpha_{2} b_{2}^{v}}$ \\
$\mathbf{2}$ & 0 & 0 & $\alpha_{2} b_{2}^{v}$ \\
\hline
\end{tabular}

By considering all the permutations of the grand coalition, the average payoff of players in this model is given as

$$
\sum_{\pi \in \Pi(N)} \frac{1}{n !} \eta_{i}^{\vec{\alpha} \pi}=\alpha_{i} b_{i}^{v}+\frac{v(N)-\sum_{j \in N} \alpha_{j} b_{j}^{v}}{3}, \forall i \in N .
$$


In view of equation (3.9), we have

$$
v^{2}(S)= \begin{cases}v(N), & \text { if } S=\{2,3\} \\ v(S), & \text { otherwise }\end{cases}
$$

and

$$
v^{3}(S)= \begin{cases}v(N), & \text { if } S=\{2,3\},\{1,3\}, \\ v(S), & \text { otherwise. }\end{cases}
$$

According to the formula in Equation (3.8), we can obtain the average payoff for players as follows

$$
\sum_{\pi \in \Pi(N)} \frac{1}{n !} \eta_{i}^{\vec{\alpha}} \pi=\alpha_{i} b_{i}^{v}+\frac{v(N)-\sum_{j \in N} \alpha_{j} b_{j}^{v}}{3}, \forall i \in N,
$$

which is the same as in Equation (3.10).

\subsection{Optimization implementation of the $\alpha$-ENSC value}

The nucleolus and the least square pre-nucleolus both are the solutions for certain optimization problems of which the aims are to minimize the complaint of coalitions under different criterion. In this section, we discuss a complaint from the perspective of individual player and explore the optimal solutions for the same optimization models as in the determination of nucleolus and the least square pre-nucleolus based on the new complaint we define.

Definition 3.4. Given that players are all $\alpha$ egoistic and the partially compromised ideal payoff $\eta_{i}^{\alpha \pi}$ is in the form of (7.2), then denote $e\left(i, \mathbf{x}, \eta^{\alpha \pi}\right)$ the complaint of player $i$ at the payoff vector $\mathbf{x}$ with respect to the ideal payoff $\eta^{\alpha \pi}$ of the game $v$, i.e.,

$$
e\left(i, \mathbf{x}, \eta^{\alpha \pi}\right)=\eta_{i}^{\alpha \pi}-\mathbf{x}_{i} \quad i \in N, \mathbf{x} \in I^{*}(v) .
$$

Recall that the least square pre-nucleolus is the pre-imputation payoff for which the complaint vector is closest to vector zero. One may wonder that 
what will the optimal solution be when considering the new complaint of individual players with the similar optimization model. Formally, the following problem is taken into account so as to minimize the variance of complaint for players.

\section{Problem 3.1.}

$$
\begin{aligned}
& \min \sum_{i \in N} \sum_{\pi}\left(\eta_{i}^{\alpha \pi}-\mathbf{x}_{i}\right)^{2} \\
& \text { s.t. } \quad \sum_{i \in N} \mathbf{x}_{i}=v(N), \mathbf{x} \in \mathbf{R}^{n} .
\end{aligned}
$$

Theorem 3.3. For any $(N, v) \in \Gamma_{n}$, there exists a unique solution $\boldsymbol{x}^{*} \in \mathbf{R}^{n}$ of Problem 3.1, which coincides with the $\alpha$-ENSC value, i.e.,

$$
\boldsymbol{x}^{*}=\alpha-\operatorname{ENSC}(v)=\alpha \operatorname{ENSC}(v)+(1-\alpha) \operatorname{ED}(v)
$$

According to Theorem 3.1, since the $\alpha$-ENSC value is an average ideal payoff over all the orders, it is clear that it minimizes the variance in Problem 3.1. Thus, Theorem 3.3 is straightforward.

Definition 3.5. Given that players are all $\alpha$ partially egoistic, denote $e^{*}(i, \mathbf{x})$ the expected complaint of $i$ at the payoff vector $\mathbf{x}$ by considering $n$ ! different coalition formation orders in complaint criterion (3.14), i.e., for all $i \in N$, $\mathbf{x} \in I^{*}(v)$

$$
e^{*}(i, \mathbf{x})=\sum_{\pi} \frac{1}{n !}\left[\eta_{i}^{\alpha \pi}-\mathbf{x}_{i}\right] .
$$

One may notice that all players have the same expected complaints with respect to the allocation under the $\alpha$-ENSC value. Given expected complaint criterion in form (3.16), a value $\phi$ satisfies

- Equal expected complaint property: if $e^{*}(i, \phi)=e^{*}(j, \phi)$, for any $i, j \in N$.

Obviously, the equal expected complaint property and efficiency define the $\alpha$-ENSC value. 
The nucleolus [71] is an optimal value which minimizes the complaint function in the lexicographic order over the imputation set. Given the expected payoff complaint criterion in the form of (3.16), we define the socalled expected complaint vector $\theta^{*}(\mathbf{x}) \in \mathbf{R}^{n}$, for which components are the expected complaints $e^{*}(i, \mathbf{x})$ arranged in non-increasing order. The next theorem states that the $\alpha$-ENSC value is the solution that minimizes the expected complaint vector in lexicographic order among pre-imputation set.

Theorem 3.4. For any $v \in \Gamma_{n}$, the $\alpha$-ENSC value is the unique pre-imputation that satisfies

$$
\theta^{*}(\alpha-\operatorname{ENSC}(v)) \leq_{L} \theta^{*}(x) \quad \text { for all } \boldsymbol{x} \in I^{*}(v)
$$

Where $\leq_{L}$ is the lexicographic order.

Proof. Given any $\mathbf{x} \in I^{*}(v)$, the expected complaint of player $i$ is

$$
e^{*}(i, \mathbf{x})=\sum_{\pi} \frac{1}{n !}\left[\eta_{i}^{\alpha \pi}-\mathbf{x}_{i}\right]=\alpha-E N S C_{i}(v)-\mathbf{x}_{i} .
$$

The last equation is derived from Theorem 3.1. Moreover, $\sum_{i \in N} \alpha-E N S C_{i}(v)=$ $\mathbf{x}(N)=v(N)$, thus for any $\mathbf{x} \in I^{*}(v)$, it holds that $\sum_{i \in N} \theta_{i}^{*}(\mathbf{x})=0$. Together with $\theta^{*}(\alpha-\operatorname{ENSC}(v))=(0,0, \cdots, 0)$, we can obtain that the $\alpha$-ENSC value is the unique pre-imputation in the above conditions satisfying the lexicographic order.

\subsection{Characterization of the $\alpha$-ENSC value}

van den Brink and Funaki [89] applied dual individual rationality to characterize the ENSC value. Dual individual rationality indicates that players will get at least his marginal contribution to the grand coalition for dual weakly essential games, which states that the total grand marginal contribution for players does not exceed the worth of the grand coalition. Formally, a value $\phi$ satisfies

- Dual individual rationality: if $\phi_{i}(v) \geq v_{*}(i)$, for all $i \in N$ and dual weakly essential game $(N, v) \in \Gamma_{n}$ (that is $\sum_{j \in N} v_{*}(j) \leq v(N)$ ). 
But as we have assumed that players are all $\alpha$ egoistic, which implies that their grand marginal contributions may no longer be the objective for players, so the following property is put forward. A value $\phi$ satisfies

- $\alpha$-dual individual rationality: if $\forall i \in N, \phi_{i}(v) \geq \alpha[v(N)-v(N \backslash i)]$, for any $\alpha$-dual weakly essential game $(N, v) \in \Gamma_{n}$.

Game $v$ is $\alpha$-dual weakly essential if $\alpha \sum_{i \in N}[v(N)-v(N \backslash i)] \leq v(N), \alpha \in$ $[0,1]$.

The property of $\alpha$-dual individual rationality states that players could only guarantee a fraction $\alpha$ of his grand marginal contribution. However, there always exists gap between reality and ideality. This can only be realized when the worth of the grand coalition is adequate, that is, for $\alpha$-dual weakly essential game.

It is not difficult to verify that the $\alpha$-ENSC value satisfies this property. Moreover, we could also apply this property together with other properties to characterize the $\alpha$-ENSC value. In order to do that, we first consider a new subclass of $\Gamma_{n}$. For any $T \varsubsetneqq N$,

$$
Z^{T}(S)= \begin{cases}\alpha-1, & \text { if } T=N \backslash S \\ 0, & \text { if } T \varsubsetneqq N \backslash S \\ \alpha, & \text { otherwise }\end{cases}
$$

As to $T=N$, we define $Z^{N}(S)=1$, for all $S \subset N$. Besides, we consider the dual game of the standard game $b^{T}$ in form of (1.5) denoted by $b_{*}^{T}(S)=$ $b^{T}(N)-b^{T}(N \backslash S)$, for all $S, T \subset N$, and $T \neq \emptyset$.

Theorem 3.5. The $\alpha$-ENSC value is the unique value that satisfies efficiency, linearity, symmetry and $\alpha$-dual individual rationality.

Proof. One could easily verify that the $\alpha$-ENSC value satisfies the four properties. The remaining part is to prove the uniqueness. Suppose that solution $\phi$ obeys above mentioned properties. 
Given a coalition $T$, consider cooperative game $\left(N, Z^{T}\right)$ in (3.17). When $|T|=1, \alpha \sum_{i \in N}\left[Z^{T}(N)-Z^{T}(N \backslash i)\right]=\alpha[n \alpha-(\alpha-1)-(n-1) \alpha]=\alpha=Z^{T}(N)$, that is, game $Z^{T}$ is $\alpha$-dual weakly essential. Thus the $\alpha$-dual individual rationality implies that

$$
\phi_{i}\left(Z^{T}\right) \geq \alpha\left[Z^{T}(N)-Z^{T}(N \backslash i)\right]= \begin{cases}\alpha, & \text { if } i \in T \\ 0, & \text { if } i \notin T\end{cases}
$$

Together with efficiency principle $\sum_{i \in N} \phi_{i}\left(Z^{T}\right)=Z^{T}(N)=\alpha$, we have

$$
\phi_{i}\left(Z^{T}\right)= \begin{cases}\alpha, & \text { if } i \in T \\ 0, & \text { if } i \notin T\end{cases}
$$

When $2 \leq|T| \leq n-1$, observe that $\alpha \sum_{i \in N}\left[b_{*}^{T}(N)-b_{*}^{T}(N \backslash i)\right]=0=$ $b_{*}^{T}(N)$, the $\alpha$-dual individual rationality implies that $\phi_{i}\left(b_{*}^{T}\right) \geq \alpha\left[b_{*}^{T}(N)-\right.$ $\left.b_{*}^{T}(N \backslash i)\right]=0, \forall i \in N$. Besides, efficiency principle states that $\sum_{i \in N} \phi_{i}\left(b_{*}^{T}\right)=$ $b_{*}^{T}(N)=0$, thus $\phi_{i}\left(b_{*}^{T}(N)\right)=0$, for all $i \in N$.

When $T=N$, since all players are symmetric in game $b_{*}^{N}$, symmetry and efficiency principle imply that $\phi_{i}\left(b_{*}^{N}\right)=\frac{b_{*}^{N}(N)}{n}=\frac{1}{n}$, for all $i \in N$.

From above discussion, we can see that solution $\phi$ is unique for game $Z^{T}$ with $|T|=1$ and $b_{*}^{T}$ with $|T| \geq 2$. The last part we have to prove is that the set $\left\{Z^{T}\right\}_{T \subset N,|T|=1} \cup\left\{b_{*}^{T}\right\}_{T \subset N,|T| \geq 2}$ forms a basis of $\Gamma_{n}$.

Denote all non-empty subsets of $N$ by $S_{1}, S_{2}, \cdots, S_{2^{n}-1}$ which are arranged in lexicographic order as discussed by Xu et al. [98]. Let $\mathbf{C}=\left[c_{i j}\right]$ be a $2^{n}-1$ square matrix defined by

$$
c_{i j}= \begin{cases}Z^{S_{i}}\left(S_{j}\right), & \text { if }\left|S_{i}\right|=1 \\ b_{*}^{S_{i}}\left(S_{j}\right), & \text { if }\left|S_{i}\right| \geq 2\end{cases}
$$

Observe that when $n<i<2^{n}-1$, there is only one nonzero element(which equals -1) in row $i$ and all elements of the last row are equal to 1 . By expanding the matrix in these rows step by step, we have $|\operatorname{det} \mathbf{C}|=\left|\operatorname{det} \mathbf{C}^{\prime}\right|$, where $\mathbf{C}^{\prime}=\left[c_{i j}^{\prime}\right]_{(n+1) \times(n+1)}$ is the final cofactor of matrix $\mathbf{C}$. The specific form of $\mathbf{C}^{\prime}$ 
is

$$
c_{i j}^{\prime}= \begin{cases}\alpha-1, & \text { if } i+j=n+1 \\ 1, & \text { if } i=n+1 \\ \alpha, & \text { otherwise }\end{cases}
$$

By simple calculation, we find that $|\operatorname{det} \mathbf{C}|=\left|\operatorname{det} \mathbf{C}^{\prime}\right|=1 \neq 0$. So we conclude that $\left\{Z^{T}\right\}_{T \subset N,|T|=1} \cup\left\{b_{*}^{T}\right\}_{T \subset N,|T| \geq 2}$ forms a basis of $\Gamma_{n}$. Applying the linearity of $\phi$, we get the conclusion that solution $\phi$ is unique for any game $v \in \Gamma_{n}$, and it is just the $\alpha$-ENSC value.

We now introduce other two properties to characterize the $\alpha$-ENSC value called $\alpha$-inessential game property and grand marginal contribution monotonicity respectively. A value $\phi$ satisfies

- $\alpha$-egocentric inessential game property: if $\forall i \in N, \phi_{i}(v)=\alpha v(i)+$ $(1-\alpha) \frac{v(N)}{n}, \alpha \in[0,1]$, for any inessential game.

A game $(N, v) \in \Gamma_{n}$ is inessential if $v(S)=\sum_{i \in S} v(i), \forall S \subset N$. When a game is inessential, the general consideration is that players have no association with others and act alone, so a proper value allocates total worth according to their own power, that is, $v(i)$ for all $i \in N$. Such idea bases on the assumption that players are all totally egocentric. But as discussed earlier, player may be not that egocentric, they could also behave altruistically. $\alpha$-egocentric inessential game property, in some sense, reflects the partial egoism. Together with efficiency and grand marginal contribution monotonicity, as introduced in Chapter 2, $\alpha$-egocentric inessential game property could fully characterize the $\alpha$-ENSC value.

Theorem 3.6. The $\alpha$-ENSC value is the unique value that satisfies efficiency, linearity, $\alpha$-egocentric inessential game property and grand marginal contribution monotonicity.

Proof. It is clear that the $\alpha$-ENSC value satisfies the four properties. It remains to prove the uniqueness part.

For any $(N, v) \in \Gamma_{n}$, suppose that $\phi$ is a value with the four mentioned properties. We now construct a new game $w \in \Gamma_{n}$ defined as $w(S)=v(S)-$ 
$\sum_{j \in S}[v(N)-v(N \backslash j)], \forall S \subset N$. Denote $v^{0}=v-w$, then one could verify that game $v^{0}$ is an inessential game. Applying $\alpha$-egocentric inessential game property, we have $\phi_{i}\left(v^{0}\right)=\alpha v^{0}(i)+(1-\alpha) \frac{v^{0}(N)}{n}$, for all $i \in N$. Further, $w(N)-w(N \backslash i)=w(N)-w(N \backslash j), \forall i, j \in N$. According to grand marginal contribution monotonicity, we have $\phi_{i}(w)=\phi_{j}(w)$, for all $i, j \in N$. Since $\phi$ also obeys efficiency principle, then $\phi_{i}(w)=\frac{w(N)}{n}, \forall i \in N$. Eventually, linearity implies that $\phi(v)=\phi(w)+\phi\left(v^{0}\right)$, which is equal to the $\alpha$-ENSC value.

\subsection{Bidding mechanism design for the $\alpha$-ENSC value}

The methods of axiomatisations of the solution concepts of cooperative games have been sufficiently studied in literature. On the one hand, solution concepts can be characterised by a set of properties. On the other hand, researchers also implement solution concepts in a non-cooperative way. Solution concepts turn to be the outcomes, namely equilibria, of some noncooperative games, which are usually called bidding mechanism. The fundamental skeleton of the bidding mechanism is that players first bid for the proposer who takes charge of the potential offer to other players. When an offer is made by the proposer, it can be accepted or rejected by the others. In case it is accepted, players except the proposer will obtain the proposed offers and the proposer get the remainder. Once the offer is rejected, the proposer has to act alone or withdraw from the bidding process with some payoff and the other players continue to play the bidding process with some new proposer. Such bidding method has been investigated in different situations. Crawford [17] generated Pareto-efficient egalitatian-equivalent allocations by introducing a bidding procedure. Evans [27] implemented the core in subgame perfect equilibria (SPE for short) by using bidding step of the discrete time non-cooperative coalitional bargaining games. To implement the Shapley value, Perez-Castrillo and Wettstein [61, 62]constructed a bidding mechanism, in which players will first bid for the proposer before the proposal step. Player with the highest net bid will be the proposer and this bid has to be paid to the others before they go to the next step. Following the 
work of Perez-Castrillo and Wettstein [61,62], Ju and Wettstein [42] implemented a continuum of cooperative solution concepts, the $\alpha$-consensus value, by introducing the second round of proposals.

Inspired by the idea, we now provide a bidding mechanism to implement the $\alpha$-ENSC value. The primary difference from the previous mechanism is the treatment of the rejected proposer. As we have argued in former sections, players aspire to get a proportion of their marginal contributions to the grand coalition. Once the proposal of some proposer is rejected, he or she has to leave the game. It seems greedy if the abandoned player still charges his grand marginal contribution. Instead of assigning the individual worth to the rejected proposer, a discounted grand marginal contribution, parameterised by $\alpha \in[0,1]$, will be allocated to the player. Meanwhile, the fraction $1-\alpha$ can also be regarded as the tax imposed on the proposer due to his unacceptable proposal. Formally, the bidding mechanism is stated as follows

Mechanism 1: For games with only one player $i$, he obtains $v(i)$. For games with the player set $N=\{1,2, \cdots, n\}$, the mechanism begins with the set $S=N$.

Stage 1: Every player $i \in S$ makes a bid $b_{j}^{i} \in \mathbb{R}$ to player $j \in S \backslash\{i\}$. The net bid for player $i$ is the difference between the total bid he makes to others and total bid the other players make to him, i.e., $B^{i}=\sum_{j \in S \backslash\{i\}} b_{j}^{i}-\sum_{j \in S \backslash\{i\}} b_{i}^{j}$. Denote $i_{s}=\arg \max _{i \in S}\left\{B^{i}\right\}$ as the player with the highest net bid. In case there are more than one maximiser, we choose one player arbitrarily. Then $i_{S}$ plays the role as the proposer and pays other players his bid $b_{j}^{i}$.

Stage 2: Player $i_{S}$ proposes an allocation scheme $x_{j}^{i_{s}} \in \mathbb{R}$ to $j \in S \backslash\{i\}$.

Stage 3: Players in $S \backslash\{i\}$ sequentially make their decisions whether they are willing to accept the offer made by $i_{s}$ in Stage 2. The offer is accepted only when all players accept it. Otherwise, it is rejected.

The game ends when a proposed offer is accepted. If the offer in stage 3 is rejected, the proposer $i_{s}$ leaves the game with the discounted grand marginal contribution $\alpha b_{i_{s}}^{v}$. The other payers in $S \backslash\{i\}$ keep going through the mechanism from stage 1 to 3 and bargain over $v(S)-\alpha b_{i_{s}}^{v}$. In this way, the final payoff for $i_{s}$ is $\alpha b_{i_{s}}^{v}-\sum_{j \in S \backslash\left\{i_{s}\right\}} b_{j}^{i_{s}}+\sum_{t=s+1}^{n} b_{i_{s}}^{i_{t}}$. 
If the offer in stage 3 is accepted, the final payoff for players depend on the size of the coalition $S$. When $S=N$, players reach a consensus, and the whole game ends. Player $i_{n}$ obtains $v(N)-\sum_{j \in N \backslash\left\{i_{n}\right\}} x_{j}^{i_{n}}-\sum_{j \in N \backslash\left\{i_{n}\right\}} b_{j}^{i_{n}}$, and every player $j \in N \backslash\left\{i_{n}\right\}$ receives $x_{j}^{i_{n}}+b_{j}^{i_{n}}$. When $S \neq N$, this game still terminates. Any player $j \in N \backslash\left\{i_{s}\right\}$ receives the final payoff $x_{j}^{i_{s}}+\sum_{t=s}^{n} b_{j}^{i_{t}}$. Player $i_{s}$ receives $v\left(S \cup i_{s+1}\right)-\alpha b_{i_{s+1}}^{v}-\sum_{j \in S \backslash\left\{i_{s}\right\}} x_{j}^{i_{s}}-\sum_{j \in S \backslash\left\{i_{s}\right\}} b_{j}^{i_{s}}+\sum_{t=s+1}^{n} b_{i_{s}}^{i_{t}}$.

Theorem 3.7. For any cooperative game $v \in \Gamma_{n}$, the $\alpha$-ENSC value is the SPE outcome of Mechanism 1.

Proof. Note that the difference between our proof and the proof of Theorem 1 in [61] is the strategy designed to generate the $\alpha$-ENSC value. Here we only concentrate on the construction of an SPE. The proof is based on the induction of the size of player set. Theorem 3.7 holds when $n=1$. We assume that it is always true for any cooperative game with $m<n$ players. Hence, we will show the validity for $n$.

First we consider the following strategy, which will be enforced by all the players whichever subgame they are playing. Here we only descirbe the case for $N$, but these strategies are also followed by players in any player set $S \neq N$.

In Stage 1, every player i bid $b_{j}^{i}=\alpha-E N S C_{j}(v)-\alpha-E N S C_{j}\left(N \backslash\{i\}, v^{-i}\right)$ to any $j \in N \backslash\{i\}$, where the game $\left(N \backslash\{i\}, v^{-i}\right)$ is given by $v^{-i}(S)=v(S \cup i)-\alpha b_{i}^{v}$ for all $S \subset N \backslash\{i\}$.

In Stage 2, player $i_{n}$ offers $\alpha$-ENSC $C_{j}\left(N \backslash\{i\}, v^{-i_{n}}\right)$ to any $j \in N \backslash\left\{i_{n}\right\}$.

In Stage 3, players in $N \backslash\left\{i_{n}\right\}$ accept the offer if it's not less than the amount $\alpha-E N S C_{j}\left(N \backslash\left\{i_{n}\right\}, v^{-i_{n}}\right)$. And they will reject the offer which is strictly less than $\alpha-E N S C_{j}\left(N \backslash\{i\}, v^{-i_{n}}\right)$.

Obviously, any non-proposer player will receive his $\alpha$-ENSC value since acceptance will occur in stage. And the proposer will also obtain his $\alpha$-ENSC due to the formation of the grand coalition and the efficiency of the $\alpha$-ENSC value. Furthermore, the above strategy constitutes an SPE, which can be prove in a similar way in [61]. Here we only prove that the net bids for 
players are the same and equals zero.

$$
\begin{aligned}
B^{i} & =\sum_{j \in N \backslash\{i\}} b_{j}^{i}-\sum_{j \in N \backslash\{i\}} b_{i}^{j} \\
& =\sum_{j \in N \backslash\{i\}}\left[\alpha-E N S C_{j}(v)-\alpha-E N S C_{j}\left(N \backslash\{i\}, v^{-i}\right)\right] \\
& -\sum_{j \in N \backslash\{i\}}\left[\alpha-E N S C_{i}(v)-\alpha-E N S C_{i}\left(N \backslash\{j\}, v^{-j}\right)\right] \\
& =\sum_{j \in N \backslash\{i\}}\left[\alpha b_{j}^{v}+\frac{v(N)-\alpha \sum_{k \in N} b_{k}^{v}}{n}-\alpha b_{j}^{v^{-i}}-\frac{v^{-i}(N \backslash\{i\})-\alpha \sum_{k \in N \backslash\{i\}} b_{k}^{v^{-i}}}{n-1}\right] \\
& -\sum_{j \in N \backslash\{i\}}\left[\alpha b_{i}^{v}+\frac{v(N)-\alpha \sum_{k \in N} b_{k}^{v}}{n}-\alpha b_{i}^{v^{-j}}-\frac{v^{-j}(N \backslash\{j\})-\alpha \sum_{k \in N \backslash\{j\}} b_{k}^{v^{-j}}}{n-1}\right] \\
& =0-0=0 .
\end{aligned}
$$

The last equality holds since $b_{j}^{v^{-i}}=b_{j}^{v}$ for any $j \in N \backslash\{i\}$ and $v^{-i}(N \backslash\{i\})=$ $v(N)-\alpha b_{i}^{v}$ for any $i \in N$.

The fact that any SPE implements the $\alpha$-ENSC value can be verified by means of some claims, which are natural modifications of the claims in PerezCastrillo and Wettstein's work. Here we list these claims and provide the proof of the last one.

Claim 1 In any SPE, at stage 3 players accept the offer when $x_{j}^{i_{n}}>\alpha$ $E N S C_{j}\left(N \backslash\left\{i_{n}\right\}, v^{-i_{n}}\right)$ for all $j \in N \backslash\left\{i_{n}\right\}$. Otherwise, the offer is rejected.

Claim 2 For the subgame that starts at stage 2, there are two SPE. One is that the proposer $i_{n}$ gives the offer as $x_{j}^{i_{n}}=\alpha-E N S C_{j}\left(N \backslash\{i n\}, v^{-i_{n}}\right)$ for all $j \in$ $N \backslash\left\{i_{n}\right\}$, and at stage 3 , every player accepts the offer $x_{j}^{i_{n}}>\alpha$-ENSC $C_{j}\left(N \backslash\left\{i_{n}\right\}, v^{-i_{n}}\right)$ and rejects the offer otherwise. The other SPE is that at stage $2 i_{n}$ offers $x_{j}^{i_{n}} \leq \alpha-E N S C_{j}\left(N \backslash\left\{i_{n}\right\}, v^{-i_{n}}\right)$ to some player $j \in N \backslash\left\{i_{n}\right\}$ and at stage 3 , players reject the offer that satisfies $x_{j}^{i_{n}} \leq \alpha-E N S C_{j}\left(N \backslash\left\{i_{n}\right\}, v^{-i_{n}}\right)$.

Claim 3 In any SPE, all the net bids are the same, therefore $B^{i}=0$ for all $i \in N$.

Claim 4 In any SPE, the final payoff for any player is independent of the choice of the proposer. 
Claim 5 In any SPE, the final payoff for each player is exactly the $\alpha$-ENSC value.

Proof. Note that the final payoff to $i$ as a proposer is $\alpha b_{i}^{v}-\sum_{j \neq i} b_{j}^{i}$. If $j \neq i$ is the proposer, the final payoff for $i$ is given by $\alpha-E N S C_{i}\left(N \backslash\{j\}, v^{-j}\right)+b_{i}^{j}$. Thus the total final payoffs over all potential proposers is

$$
\begin{aligned}
\alpha b_{i}^{v} & -\sum_{j \neq i} b_{j}^{i}+\sum_{j \neq i}\left[\alpha-E N S C_{i}\left(N \backslash\{j\}, v^{-j}\right)+b_{i}^{j}\right] \\
& =\alpha b_{i}^{v}+\sum_{j \neq i} \alpha-E N S C_{i}\left(N \backslash\{j\}, v^{-j}\right) \\
& =\alpha b_{i}^{v}+\sum_{j \neq i}\left[\alpha b_{i}^{v}+\frac{v(N)-\alpha b_{j}^{v}-\alpha \sum_{k \neq j} b_{k}^{v}}{n-1}\right] \\
& =n \alpha b_{i}^{v}+v(N)-\alpha \sum_{k \in N} b_{k}^{v} \\
& =n \alpha-\operatorname{ENSC}_{i}(v) .
\end{aligned}
$$

The first equality is derived from Claim 3. By Claim 4, the final payoff is regardless of the proposer, thus the final payoff must coincide with the $\alpha$ ENSC value. 



\section{Chapter 4}

\section{Responsibility and sharing the cost of cleaning a polluted river}

Consider $n$ firms (agents) located at a river, indexed by $1, \ldots, n$ from upstream to downstream. The pollution generated by these firms induce cleaning costs $c_{1}, \ldots, c_{n}$, where $c_{i}$ is the cost for cleaning the water in region $i$ (according to the local environmental standards). The corresponding cost allocation problem is highly interesting both in theory and practice. Among the most prominent allocation schemes are the so-called Local Responsibility and Upstream Equal Sharing. The first one allocates simply each local cost $c_{i}$ to the corresponding firm $i$ located in region $i$. The second distributes each $c_{i}$ equally among firms $1, \ldots, i$. We propose and characterize a dynamic scheme which, given a particular order of arrival, allocates the current total cost among the firms that have arrived so far. The corresponding expected allocation (w.r.t. a random arrival order) turns out to be a convex combination of the two schemes above.

\subsection{Introduction}

More than 200 rivers around the world flow across different countries (see [3] and [7]) and many more rivers flow through different regions. On one hand, 
inhabitants or firms along the river can utilize the water resources. On the other hand, they may also discharge household or industrial waste into the rivers. Thus sharing a river from the aspects of both rights and responsibility is always a highly interesting issue in society.

On the beneficial side, the main aim is to find a fair allocation of the welfare resulting from distributing the water flow. Ambec et al. [3] first model such situation as a cooperative coalitional game, then they propose the downstream incremental method as a compromise between two precepts: Absolute Territorial Sovereignty [30] and Unlimited Territorial Integrity [45]. Ambec et al. [2] consider a more generalised model with satiable agents. Khmelnitskaya [44] investigates the situation where river basins possess multiple springs. van den Brink et al. [94] also study such river system by taking account of externalities.

In this chapter, we concentrate on the responsibility side of sharing a river. Agents situated along a river benefit from the water resources, but like every coin has two sides, their production processes are accompanied by pollution. There is no doubt that waste can destroy the ecosystem of the river and sometimes even cause natural disasters. Many countries around the world seek to solve the problem of river pollution. For instance, the government of China has established a chief river regulation system coordinating the various involved departments to improve the quality of China's waterways. An important issue is the question how the cost of cleaning the water should be shared among the agents involved.

$\mathrm{Ni}$ and Wang [55] first analysed the river responsibility sharing problem among different segments from a theoretical point of view. In their model, agents (polluters) are located along a river from upstream to downstream and there is an authority that determines how the total cleaning cost should be allocated to the agents. Ni and Wang [55] introduce two allocation methods, Local Responsibility Sharing (LRS for short) method and Upstream Equal Sharing (UES for short) method. These two methods are proposed under the principles of Local Responsibility and Downstream Responsibility respectively. The LRS method prescribes that each agent should take full responsibility of the cost in its area. In contrast, the UES method forces upstream agents to take some responsibility for its downstream cleaning costs - after all, it is 
often difficult to determine exactly who is responsible for which part of the pollution. More precisely, the UES method allocates the cleaning cost in a segment equally among the corresponding firm and all its upstream companies.

Alcalde-Unzu et al. [1] argued that neither LRS nor UES makes sense for all scenarios and modified the model by introducing the so-called transfer rate, which measures the proportion of the waste that is transferred from one segment of the river to its downstream. They argued that the LRS method ignored the fact that water in a river would flow from upstream to downstream with certain rate, taking some of the waste along with it. As to the UES method, although it takes into consideration the transfer rate of the pollution, the assumption that a firm in a given segment and all the firms located from its upstream have the same responsibility for the cleaning cost in this region does not make sense for all scenarios. Hence they proposed the so-called Upstream Responsibility method by taking into account the responsibility of the segments for the flow of the pollution. In many cases, this transfer rate is difficult to estimate, however. In this chapter, we therefore propose a somewhat different approach: instead of estimating the transfer rate, we assume that the firms agree on a minimal responsibility level $\alpha$, meaning that each firm would be happy to pay for an $\alpha$ fraction of the cleaning cost in its segment. All agents will agree on this minimal responsibility level. Alternatively, we can say that the minimal responsibilities are the maximal cost that agents will accept without complaint ( Of course, the value of $\alpha$ would also reflect the general opinion on a lower bound for the transfer rate.). Such an agreement may be determined from empirical data, by bargaining or simply government intervention.

Furthermore, inspired by the work of Malawski [47] and Sun et al.( [79] and [80]), we investigate the allocation method from a dynamic perspective. More specifically, suppose that the firms arrive at the river one by one. When an agent joins the river system, it is ready to pay for its minimal responsibility. Apart from this minimal responsibility, the remaining cost should also be allocated properly. As we have discussed, the upstream agents that have appeared should take the responsibility (Note that downstream agents have no obligation to contribute towards the cleaning cost at upstream locations 
in our model). To avoid potential uncertainy, every new entrant may want to allocate the cost in its area as long as it joins the procedure, but not wait until its upstream agents join. That is the reason why we assume that an agent shares in the cost of a downstream agent that enters after it but not when it enters after this downstream agent. Proceeding this way, the total cost is shared among the agents given their order of arrival. Of course, the final outcome will depend on the minimal responsibility level $\alpha$.

In case all firms are already present, we may define a corresponding expected cost allocation by randomizing over all possible arrival orderings. This defines a new sharing method which we call $\alpha$-responsibility method. Interestingly, the $\alpha$-responsibility method coincides with a convex combination of the LRS and UES method. So the $\alpha$-responsibility in some sense reconciles the two doctrines, Local Responsibility and Downstream Responsibility.

$\mathrm{Ni}$ and Wang [55] characterize the LRS and UES method with Additivity, while van den Brink et al. [92] introduce axiomatizations of these two methods without Additivity. We show that the $\alpha$-responsibility method can also be fully characterized with or without additivity. Several new properties, including Weakly Blind Cost, Surplus Monotonicity, Essential Player Property, $\alpha$-Blind Cost, and Upstream (Strong) Surplus Symmetry are proposed to axiomatically characterize the $\alpha$-responsibility method.

\subsection{Preliminaries}

Consider $n$ firms (agents) located along a river, indexed by $N=\{1,2, \cdots, n\}$ from upstream to downstream. Each of the firms generates some pollutants, which will influence the quality of the waterbody. In order to clean the pollutants according to (local) environmental standards, firms along the river have to pay for the cleaning costs. To this end, the environmental authority fixes a corresponding cost vector $c=\left(c_{1}, c_{2}, \cdots, c_{n}\right)$, where $c_{i}$ denotes the cost incurred to clean the river in segment $i$. Formally, denote such river sharing system as a pair $(N, c)$ and the class of all sharing systems with $n$ firms as $\Delta_{n}$.

Given a river sharing system $(N, c)$, the authority has to allocate the total cost along the whole river to all firms in a reasonable way. A sharing method 
is a map $x:(N, c) \rightarrow R^{n}$, and $x_{i}$ denotes the cost firm $i$ has to pay. Researchers have proposed various allocation methods to allocate the total cost $c(N)=$ $\sum_{i \in N} c_{i}$. Among these methods, the so-called Local Responsibility Sharing (LRS for short) method and Upstream Equal Sharing (UES for short) method are the most prominent ones. The LRS method states that any firm in segment $i$ should take full responsibility to the cost of cleaning in that segment, i.e.,

Definition 4.1. For any river sharing system $(N, c) \in \Delta_{n}$, the Local Responsibility Sharing method is defined by

$$
x_{i}^{L R S}(c)=c_{i}, \quad \forall i \in N
$$

The Upstream Equal Sharing method allocates each $c_{i}$ equally among firms $1, \ldots, i$ (see Section 4.1 for motivation):

Definition 4.2. For any river sharing system $(N, c) \in \Delta_{n}$, the Upstream Equal Sharing method is defined by

$$
x_{i}^{U E S}(c)=\frac{1}{i} c_{i}+\frac{1}{i+1} c_{i+1}+\cdots+\frac{1}{n} c_{n}, \quad \forall i \in N .
$$

\subsection{Dynamic implementation of the sharing method and the $\alpha$-responsibility method}

When the transfer of the pollutants is involved, no firm is willing to pay all the cleaning cost in its area, since firms from upstream also contribute to the cost in its region. But in general, it's difficult to measure the exact transfer rate technically. Instead of estimating the transfer rate as Joerge et al. [1] did, we investigate an allocation based on the minimal responsibility of the firms. To measure the minimal responsibility, we introduce a parameter $\alpha \in[0,1]$, which determines the fraction of the local cost a firm is willing to pay. When it comes to such cost allocation situations, firms definitely want to pay as little as possible. However, the minimal responsibility $\alpha c_{i}$ in some sense sets the limitation for $i$.This fraction can be determined through bargaining and negotiation among firms based on empirical data or some other information. Here 
we do not discuss this issue further and simply assume that, as a consensus, all the firms will agree on this bargaining outcome.

Inspired by the concept of procedural value proposed by Marcin [47], also studied by Sun et al.( [79] and [80]), we aim to study the sharing method by analysing the formation of the grand coalition, i.e., all firms along the river. We assume that firms join the system one by one in a given formation order of $N$. Based on the above discussion, every new entrant will merely pay for its minimal cost. Next we introduce the explicit dynamic procedure and reveal the relationship between the final allocation and the responsibility level $\alpha$.

- All firms join the system under the formation order $\pi \in \Pi(N)$, and we assume that all permutations have the same probability;

- Given a new entrant $i \in N$, it will take position $\pi(i)$ and pay for its minimal responsibility i.e., $\alpha c_{i}$ in the first place. The surplus, $c_{i}-\alpha c_{i}$ is allocated evenly among the firms from its upstream that have already arrived (Note that any existing firms, which are downstream of the new entrant, have no reason to take the responsibility of the gap. Thus, in case there is no firm upstream yet, then $i$ has to take all the cost involved in this area);

- Proceeding in this way, the total $\operatorname{cost} c(N)$ is distributed among all the firms under permutation $\pi$;

- By considering all formation orders, the final allocation for the firms is the expected allocation over all $n$ ! permutations.

We now illustrate this dynamic procedure by discussing a 3-firm example with the formation order $\pi=(3,1,2)$. At the beginning, firm 3 comes. It will be charged $c_{3}$ since there is no other firm, while 1 and 2 pay nothing at the moment (see Table 4.1).

TABLE 4.1 Cost allocation when firm 3 joins in.

\begin{tabular}{llll}
\hline Firm & $\mathbf{1}$ & $\mathbf{2}$ & $\mathbf{3}$ \\
\hline Allocation & 0 & 0 & $c_{3}$ \\
\hline
\end{tabular}


Then firm 1 comes, and it has to pay $c_{1}$ since the existing firm 3 is not its upstream. Firm 2 still pays nothing in this stage (see Table 4.2).

TABLE 4.2 Cost allocation when firm 1 joins in.

\begin{tabular}{llll}
\hline Firm & $\mathbf{1}$ & $\mathbf{2}$ & $\mathbf{3}$ \\
\hline Allocation & $c_{1}$ & 0 & $c_{3}$ \\
\hline
\end{tabular}

Finally firm 2 joins. When firm 2 enters, it is willing to pay $\alpha$ part of the local cost $\alpha c_{2}$, since the cost $c_{2}$ also includes the cost of pollution transferred from the upstream. The remaining part $(1-\alpha) c_{2}$ will be allocated to firm 1 , since it is the unique upstream of firm 2 (see Table 4.3).

TABLE 4.3 Cost allocation when firm 2 joins in.

\begin{tabular}{lccc}
\hline Firm & $\mathbf{1}$ & $\mathbf{2}$ & $\mathbf{3}$ \\
\hline Allocation & $c_{1}+(1-\alpha) c_{2}$ & $\alpha c_{2}$ & $c_{3}$ \\
\hline
\end{tabular}

Summating the cost in every stage, we obtain the firms' final allocations, which are $c_{1}+(1-\alpha) c_{2}, \alpha c_{2}$ and $c_{3}$, under the permutation $\pi=(3,1,2)$.

Generally, the cost of firm $i$ under the formation order $\pi$ consists of two parts. The first is from the cost in its own region, which is

$$
B_{i}^{\alpha \pi}(c):=\left\{\begin{aligned}
c_{i}, & \text { if } i<j, \quad \forall j \in S_{i}^{\pi} ; \\
\alpha c_{i}, & \text { otherwise, }
\end{aligned}\right.
$$

where $S_{i}^{\pi}:=\{j \in N: \pi(j) \leq \pi(i)\}$ denotes the set of firm $i$ and all the firms that precede $i$.

Another part of the cost is generated by segments from $i$ 's downstream. Denote $G_{i k}^{\alpha \pi}(c)$ as the cost transferred from firm $k$ to $i$ under the formation order $\pi$. Then $G_{i k}^{\alpha \pi}(c)$ has the following form.

$$
G_{i k}^{\alpha \pi}(c):=\left\{\begin{aligned}
\frac{c_{k}-B_{k}^{\alpha \pi}}{\left|U_{k}^{\pi}\right|}, & \text { if } i \in U_{k}^{\pi} ; \\
0, & \text { otherwise, }
\end{aligned}\right.
$$


where $U_{k}^{\pi}$ is the set of all firms from upstream of $k$ in $S_{k}^{\pi}$, i.e., $U_{k}^{\pi}=\{j \in$ $\left.S_{k}^{\pi} \mid j<k\right\}$.

Let $\xi_{i}^{\alpha \pi}$ denote the cost of firm $i$ under the formation order $\pi$ with minimal responsibility level $\alpha$. Thus

$$
\begin{aligned}
\xi_{i}^{\alpha \pi} & =B_{i}^{\alpha \pi}(c)+\sum_{k=1}^{n} G_{i k}^{\alpha \pi}(c) \\
& =B_{i}^{\alpha \pi}(c)+\sum_{k>i, \pi(k)>\pi(i)} \frac{c_{k}-\alpha c_{k}}{\left|U_{k}^{\pi}\right|} .
\end{aligned}
$$

In case all firms are present already, it is common to allocate the cost according to their expected share (with the expectation taken with respect to random arrival orderings):

Definition 4.3. For any river sharing system $(N, c) \in \Delta_{n}$, the $\alpha$-responsibility method $\xi^{\alpha}$ allocates to each firm $i \in N$ the average of its cost over all permutations, i.e.,

$$
\xi_{i}^{\alpha}(c)=\sum_{\pi \in \Pi(N)} \frac{1}{n !} \xi_{i}^{\alpha \pi}
$$

Theorem 4.1. For any river sharing system $(N, c) \in \Delta_{n}$, the $\alpha$-responsibility method coincides with the convex combination of the Local Responsibility Sharing method and the Upstream Equal Sharing method with coefficient $\alpha$, i.e.,

$$
\xi^{\alpha}(c)=\alpha x^{L R S}(c)+(1-\alpha) x^{U E S}(c) .
$$

Proof. If there is no confusion, we omit $c$ in the formulas, i.e., we write $\xi^{\alpha}$ instead of $\xi^{\alpha}(c)$ throughout the paper. For any $i \in N$, we have

$$
\xi_{i}^{\alpha}=\sum_{\pi \in \Pi(N)} \frac{1}{n !} \xi_{i}^{\alpha \pi}=\sum_{\pi \in \Pi(N)} \frac{1}{n !} B_{i}^{\alpha \pi}+\sum_{\pi \in \Pi(N)} \sum_{k>i, \pi(k)>\pi(i)} \frac{1}{n !} \frac{c_{k}-\alpha c_{k}}{\left|U_{k}^{\pi}\right|} .
$$

Denote the first item on the right hand side by $A$ and the second item by $B$. If agent $i$ enters before all $j<i$ then its contribution in its own cost is $c_{i}$, and its contribution is $\alpha c_{i}$ otherwise. Since the probability of permutations where $i$ enters before all $j<i$ is equal to $\frac{(i-1) !}{i !}=\frac{1}{i}$, its expected contribution in its own cost $\mathrm{A}$ is equal to $\frac{1}{i} c_{i}+\left(1-\frac{1}{i}\right) \alpha c_{i}=\alpha c_{i}+\frac{1-\alpha}{i} c_{i}$. 
As to item B, we group the coalitions in the second sum according to the size of $U_{k}^{\pi}$ :

$$
\begin{aligned}
B & =\frac{1}{n !} \sum_{k=i+1}^{n} \sum_{\pi: \pi(k)>\pi(i)} \frac{c_{k}-\alpha c_{k}}{\left|U_{k}^{\pi}\right|} \\
& =\frac{1}{n !} \sum_{k=i+1}^{n} \sum_{m=1}^{k-1} C_{n}^{n-k}(n-k) ! C_{k-2}^{m-1} m !(k-m-1) ! \frac{(1-\alpha) c_{k}}{m} \\
& =\frac{1}{n !} \sum_{k=i+1}^{n} \sum_{m=1}^{k-1} \frac{n !}{k(k-1)}(1-\alpha) c_{k} \\
& =\sum_{k=i+1}^{n} \frac{1}{k}(1-\alpha) c_{k} .
\end{aligned}
$$

Based on above results, we conclude that for any $i \in N$,

$$
\xi_{i}^{\alpha}=A+B=\alpha x_{i}^{L R S}+(1-\alpha) x_{i}^{U E S}
$$

For $\alpha=0$, we obtain the UES method, while $\alpha=1$ results in the LRS method. Thus $\alpha$ indeed reflects the extent to which the firms take responsibility for the cleaning cost in their segment. Hence, $\alpha$-responsibility method seems to be a proper choice for the name of this allocation concept.

Remark 4.1. Actually, we could also consider a more general model, in which players have different responsibility levels. Suppose that the responsibility level for player $i$ is $\alpha_{i}$, then the final payoff under the above procedure is $\alpha_{i} c_{i}+\sum_{k=i}^{n} 1 / k\left(1-\alpha_{k}\right) c_{k}$. The proof is similar to the proof of Theorem 4.1.

Remark 4.2. In the above model we mainly consider the situation where downstream agents have no obligation to share the cost of their upstream agents. But Dong, Ni and Wang [20] proposed another interpretation of the Unlimited Territorial Integrity, which is the opposite of the downstream responsibility. In their model, the agents also have to undertake part of the cost from its upstream agents. Actually, we could also consider the upstream responsibility in our model as follows: 
- All firms join the system under the formation order $\pi \in \Pi(N)$;

- Given a new entrant $i \in N$, it will take position $\pi(i)$ and pay for its minimal responsibility i.e., $\alpha c_{i}$ in the first place. The surplus, $c_{i}-\alpha c_{i}$ is allocated evenly among the firms from its downstream that have already arrived. (Thus, in case there is no firm downstream yet, then $i$ has to take all the cost involved in this area.);

- Proceeding in this way, the total $\operatorname{cost} c(N)$ is distributed among all the firms under permutation $\pi$.

Interestingly, under such a procedure, the expected cost allocation is exactly the convex combination of the Local Responsibility Sharing method and the Downstream Equal Sharing method, proposed by Dong et al. [20].

Remark 4.3. Notice that agents in our model share in the cost of the downstream agents that enter after them. One may argue that why they don't share such cost when they enter after their downstream agents. In fact, if we allocate the surplus of an agent evenly among the agents from its upstream that haven't arrived, then similar to the above analysis, the average cost over all permutations coincides with the $\alpha$-responsibility method.

\subsection{Characterization of the $\alpha$-responsibility method}

In this section, we introduce several axiomatizations of the $\alpha$-responsibility method with or without additivity, which is a classical property in cooperative game theory. Recall that Ni and Wang [55] characterize the LRS method and UES method with the following axioms.

- Efficiency: $\sum_{i \in N} x_{i}=\sum_{i \in N} c_{i}$ for any $(N, c) \in \Delta_{n}$.

- Additivity: for any $\left(N, c^{1}\right)$ and $\left(N, c^{2}\right) \in \Delta_{n}$, it holds that $x_{i}\left(c^{1}\right)+$ $x_{i}\left(c^{2}\right)=x_{i}\left(c^{1}+c^{2}\right)$.

- No Blind Cost: $x_{i}(c)=0$ for any $(N, c) \in \Delta_{n}$ such that $c_{i}=0$.

- Independence of Upstream Costs: if $\left(N, c^{1}\right),\left(N, c^{2}\right) \in \Delta_{n}$ and $i \in N$ with $c_{k}^{1}=c_{k}^{2}, \forall k>i$, then $x_{j}\left(c^{1}\right)=x_{j}\left(c^{2}\right)$ for all $j>i$. 
- Upstream Symmetry: if $c_{j}=0$ for all $j \neq i$, then $x_{j}(c)=x_{i}(c)$ for all $j<i$.

The LRS method is known to be the only sharing method that satisfies Efficiency, Additivity and No Blind Cost [55]. The UES method is known to be the unique sharing method that satisfies Efficiency, Additivity, Upstream Symmetry and Independence of Upstream Costs [55]. In this section, we will propose some new properties to characterize the $\alpha$-responsibility method based on these axiomatizations.

\subsubsection{Characterization based on No Blind Cost property}

No Blind Cost is a kind of "null player" axiom stating that a firm with no cleaning cost in its local area does not have to contribute anything. No cleaning cost in an area does not mean that the corresponding firm produces no pollution. For example, environmental standards in a downstream country $i+1$ may well imply non-zero cleaning costs for the waste that firm $i$ produces. This motivates a somewhat weaker Blind Cost axiom as follows.

- Weakly Blind Cost: $x_{i}(c)=0$ for any $(N, c) \in \Delta_{n}$ with $c_{j}=0, \forall j \geq i$.

Weakly Blind Cost states that if there is no waste or no cost induced by waste in all the segments from its downstream including its local area, then the firm bears no cost.

For any potential sharing method $x$, the difference between $x_{i}$ and $\alpha c_{i}$ can be viewed as the extra "social responsibility" of firm $i$. The following axiom states that upstream firms are to take more social responsibilities than downstream firms.

- Surplus Monotonicity: for any $c \in R_{+}^{n}$ and $i \in N$, it holds that $x_{i}(c)-$ $\alpha c_{i} \geq \max _{j \geq i}\left\{x_{j}(c)-\alpha c_{j}\right\}$.

Consider a river sharing system in which only one segment incurs pollution, then regardless of the position of the firm, it seems reasonable that such an essential firm should bear no less responsibility than the other firms.

- Essential Player Property: for any $j \in N$, 


$$
x_{i}\left(0, \cdots, 0, c_{i}, 0, \cdots, 0\right)-\alpha c_{i} \geq x_{j}\left(0, \cdots, 0, c_{i}, 0, \cdots, 0\right)-\alpha c_{j} .
$$

Together with efficiency and additivity, these three axioms fully characterize the $\alpha$-responsibility method.

Theorem 4.2. For any $(N, c) \in \Delta_{n}$, the $\alpha$-responsibility method is the only sharing method that satisfies Efficiency, Additivity, Weakly Blind Cost, Surplus Monotonicity and Essential Player Property.

Proof. It is easy to verify that the $\alpha$-responsibility method satisfies the five properties. In order to show the uniqueness, let $x$ be a method with the mentioned properties and consider $c^{k}$ given as $c_{i}^{k}=0$ for $i \neq k$ and $c_{k}^{k}=1$. By Weakly Blind Cost, $x_{i}\left(c^{k}\right)=0$ for all $i>k$. Surplus Monotonicity implies that for all $j<k$,

$$
\begin{aligned}
& x_{j}\left(c^{k}\right)-\alpha c_{j}^{k} \geq x_{k}\left(c^{k}\right)-\alpha c_{k}^{k} \\
\Rightarrow \quad & x_{j}\left(c^{k}\right) \geq x_{k}\left(c^{k}\right)-\alpha, \quad \forall j<k .
\end{aligned}
$$

By Essential Player Property, it follows that

$$
\begin{aligned}
& x_{k}\left(c^{k}\right)-\alpha c_{k}^{k} \geq x_{j}\left(c^{k}\right)-\alpha c_{j}^{k} \\
\Rightarrow \quad & x_{k}\left(c^{k}\right)-\alpha \geq x_{j}\left(c^{k}\right), \quad \forall j<k .
\end{aligned}
$$

Thus we conclude that $x_{j}\left(c^{k}\right)=x_{k}\left(c^{k}\right)-\alpha$ for all $j<k$. Together with Efficiency, we have

$$
\sum_{i=1}^{n} x_{i}\left(c^{k}\right)=\sum_{i<k} x_{i}\left(c^{k}\right)+x_{k}\left(c^{k}\right)=\sum_{i<k}\left[x_{k}\left(c^{k}\right)-\alpha\right]+x_{k}\left(c^{k}\right)=1,
$$

which gives that $x_{k}\left(c^{k}\right)=\alpha+\frac{1-\alpha}{k}$ and $x_{i}\left(c^{k}\right)=\frac{1-\alpha}{k}$ for $i<k$. Notice that the set $\left\{c^{k}\right\}_{k=1}^{n}$ forms a basis of $R^{n}$. For any given $c \in R^{n}, c=\sum_{i \in N} c_{i} c^{i}$, together with Additivity, it holds that

$$
\begin{aligned}
x_{i}(c) & =\sum_{k \in N} c_{k} x_{i}\left(c^{k}\right) \\
& =c_{i} x_{i}\left(c^{i}\right)+\sum_{k \neq i} c_{k} x_{i}\left(c^{k}\right)
\end{aligned}
$$




$$
\begin{aligned}
& =\alpha c_{i}+\frac{1-\alpha}{i} c_{i}+\sum_{k>i}(1-\alpha) \frac{1}{k} c_{k} \\
& =\alpha c_{i}+(1-\alpha) \sum_{k=i}^{n} \frac{1}{k} c_{k}
\end{aligned}
$$

It's worth noting that the axioms of Theorem 4.2 are quite close to those of van den Brink et al. [95], but we do propose these axioms independently. Surplus monotonicity and the essential player property are variations of structural monotonicity and the necessary agent property, which correspond to $\alpha=0$. In this way, Theorem 4.2 generalizes one of the axiomatizations of the UES method given by van den Brink et al. [95].

We have argued that a firm with no cost-inducing waste in its segment may also bear some cost generated from its downstream, but the problem is how much the responsibility should be. Recall that we have introduced the minimal responsibility level $\alpha$ to measure the amount of cost that the firms are willing to bear, then the remaining should be shared among the firms from their upstream. Therefore, compared to Weakly Blind Cost, we propose a stronger property, which states that any firm with a zero cost in its segment assumes some of the cost from its downstream i.e.,

- $\alpha$-Blind Cost: $x_{i}(c)=(1-\alpha) \sum_{j>i} \frac{1}{j} c_{j}$ for any $(N, c) \in \Delta_{n}$ with $c_{i}=0$.

The strengthening of the Weakly Blind Cost property allows us to abandon Surplus Monotonicity and the Essential Player Property in Theorem 4.2. As a result, $\alpha$-Blind Cost can fully characterize the $\alpha$-responsibility method together with Efficiency and Additivity.

Theorem 4.3. For any $(N, c) \in \Delta_{n}$, the $\alpha$-responsibility method is the only method that satisfies Efficiency, $\alpha$-Blind Cost and Additivity.

Proof. It is easy to verify that the $\alpha$-responsibility method satisfies the three properties. Let $x \in R^{n}$ be a method with the mentioned properties, then we show that $x$ is uniquely determined. 
Let $c^{k}$ be defined as $c_{i}^{k}=0$ for $i \neq k$ and $c_{k}^{k}=1$. By $\alpha$-Blind Cost, it follows that

$$
x_{i}\left(c^{k}\right)=\left\{\begin{aligned}
(1-\alpha) \frac{1}{k}, & \text { if } i<k \\
0, & i>k
\end{aligned}\right.
$$

Together with Efficiency, we have $x_{k}\left(c^{k}\right)=1-(1-\alpha) \frac{k-1}{k}$. Similar to the last part of the proof of Theorem 4.2, by applying Additivity, it holds that

$$
x_{i}(c)=\alpha c_{i}+(1-\alpha) \sum_{k=i}^{n} \frac{1}{k} c_{k} .
$$

Young [102] provides an axiomatization of the Shapley value for the class of cooperative games without using Additivity but Strong Monotonicity. Van den Brink et al. [92] show that a sharing method satisfies Independence of Upstream Costs when the corresponding solution concepts obeys Strong Monotonicity and introduce a new characterization of the UES method. Following the approach of van den Brink et al., we show that the $\alpha$-responsibility method can also be fully axiomatized by replacing Additivity in Theorem 4.3 with Independence of Upstream Costs.

Theorem 4.4. For any $(N, c) \in \Delta_{n}$, the $\alpha$-responsibility method is the only method that satisfies Efficiency, $\alpha$-Blind Cost and Independence of Upstream Costs.

Proof. First of all, it is not difficult to verify that the method satisfies the three properties. It remains to show uniqueness. Let $x \in R^{n}$ be a method with the mentioned properties. We are to prove that $x$ is exactly the $\alpha$-responsibility method.

Let $c^{n} \in R_{+}^{n}$ be defined by $c_{i}^{n}=0$ for $i<n$ and $c_{n}^{n}=c_{n}$. $\alpha$-Blind Cost implies that $x_{i}\left(c^{n}\right)=\frac{(1-\alpha)}{n} c_{n}^{n}=\frac{(1-\alpha)}{n} c_{n}$ for all $i<n$. Efficiency implies that $x_{n}\left(c^{n}\right)=c_{n}-(n-1) \frac{(1-\alpha)}{n} c_{n}=\alpha c_{n}+(1-\alpha) \frac{1}{n} c_{n}$. Together with Independence of Upstream Costs, it holds that $x_{n}(c)=x_{n}\left(c^{n}\right)=\alpha c_{n}+(1-\alpha) \frac{1}{n} c_{n}$, which means that the cost for $n$ is uniquely determined. Similarly, define $c^{j}$ as $c_{i}^{j}=c_{i}$ for all $i \geq j$ and $c_{i}^{j}=0$ for all $i<j$. According to $\alpha$-Blind Cost, we have that 
$x_{i}\left(c^{j}\right)=(1-\alpha) \sum_{k=j}^{n} \frac{1}{k} c_{k}^{j}$ for all $i<j$ are uniquely determined. Together with Independence of Upstream Costs, we then deprive $x_{j}(c)=x_{j}\left(c^{j}\right)$, which is also uniquely determined.

Remark 4.4. As a matter of fact, combined with $\alpha$-Blind Cost, additivity implies Independence of Upstream Costs. For any $\left(N, c^{1}\right),\left(N, c^{2}\right) \in \Delta_{n}$ and $i \in N$ such that $c_{k}^{1}=c_{k}^{2}, \forall k>i$, it holds that $x_{j}\left(c^{1}\right)-x_{j}\left(c^{2}\right)=x_{j}\left(c^{1}-c^{2}\right)$ by additivity. With $\alpha$-Blind Cost, we have $x_{j}\left(c^{1}-c^{2}\right)=(1-\alpha) \sum_{k>j} \frac{1}{k}\left(c_{k}^{1}-c_{k}^{2}\right)=0$. Thus we conclude that $x_{j}\left(c^{1}\right)-x_{j}\left(c^{2}\right)=0$ for all $j>i$, which is the Independence of Upstream Costs property. However, the Independence of Upstream Costs is not equivalent to additivity. The sharing method $x_{i}(c)=\left[c_{i}\right]^{2}$ satisfies the Independence of Upstream Costs but not additivity, since efficiency plays a significant part in this situation.

\subsubsection{Characterization based on Upstream Symmetry}

$\mathrm{Ni}$ and Wang [55] proposed the Upstream Symmetry to capture the idea that any given downstream costs are shared evenly among the upstream firms. In our model, all the firms will reach an agreement on the minimal ratio of local costs they have to bear, thus when we deal with the responsibility of firms from upstream, it seems more fair to consider the surplus, i.e., $x_{i}-\alpha c_{i}$, where $x$ is a potential sharing method. Correspondingly, we have the following modified symmetry axiom.

- Upstream Surplus Symmetry: $x_{j}(c)-\alpha c_{j}=x_{k}(c)-\alpha c_{k}$ for all $j, k \leq i$, where $c_{j}=0$ for all $j \neq i$.

Theorem 4.5. For any $(N, c) \in \Delta_{n}$, the $\alpha$-responsibility method is the only method that satisfies Efficiency, Additivity, Upstream Surplus Symmetry and Independence of Upstream Costs.

Proof. First, the $\alpha$-responsibility method satisfies the four properties. In order to show the uniqueness, let $x$ be a solution with the mentioned properties and consider $c^{k} \in R^{n}$ defined by $c_{i}^{k}=0$ for all $i \neq k$ and $c_{k}^{k}=1$. By Independence of Upstream Costs, $x_{j}\left(c^{k}\right)=x_{j}(0,0, \cdots, 0)=0$ for all $j>k$. By Upstream $\alpha$ Symmetry, there must exist $\beta, \gamma \in R$ such that $x_{k}\left(c^{k}\right)=\gamma=x_{j}\left(c^{k}\right)+\alpha=\beta+\alpha$ 
for all $j<k$. Moreover, Efficiency implies that

$$
(k-1) \beta+\gamma=(k-1) \beta+\beta+\alpha=1,
$$

which gives that

$$
x_{i}\left(c^{k}\right)=\left\{\begin{aligned}
\frac{1-\alpha}{k}, & \text { if } i<k ; \\
\frac{1-\alpha}{k}+\alpha, & \text { if } i=k ; \\
0, & i>k .
\end{aligned}\right.
$$

Based on above consideration and Additivity, for any $c \in R^{n}$, one can easily obtain that (the details are left to the reader)

$$
x_{i}(c)=\alpha c_{i}+(1-\alpha) \sum_{k=i}^{n} \frac{1}{k} c_{k} .
$$

Upstream Surplus Symmetry implies that firm $i$ and all firms from its upstream bear the same surplus when all firms incur no cost except $i$. Now we consider a stronger axioms by weakening the condition that all firms from $i$ 's upstream incur no cost in their segments.

- Strong Upstream Surplus Symmetry: $x_{j}\left(c^{i}\right)-\alpha c_{j}^{i}=x_{k}\left(c^{i}\right)-\alpha c_{k}^{i}$ for all $j, k \leq i$, where $c_{j}^{i}=0$ for all $j<i$.

It is not difficult to show that Upstream Surplus Symmetry and Additivity imply Strong Upstream Surplus Symmetry. In fact, compared with Theorem 4.5 , this property allows us to get rid of Additivity.

Theorem 4.6. For any $(N, c) \in \Delta_{n}$, the $\alpha$-responsibility method is the only method that satisfies Efficiency, Strong Upstream Surplus Symmetry and Independence of Upstream Costs.

Proof. One can easily verify that the $\alpha$-responsibility method obeys the properties. It remains to prove the uniqueness. Suppose $x$ is a method with the three properties. Consider $c^{n} \in R^{n}$ defined by $c_{i}^{n}=0$ for $i<n$ and $c_{n}^{n}=c_{n}$. By Upstream Strong Surplus Symmetry, there exist $\beta, \gamma \in R$ such 
that $x_{i}\left(c^{n}\right)=\beta=x_{n}\left(c^{n}\right)-\alpha c_{n}^{n}=\gamma-\alpha c_{n}^{n}$. Together with Efficiency, it holds that

$$
\sum_{i \in N} x_{i}\left(c^{n}\right)=(n-1) \beta+\gamma=c_{n}^{n},
$$

which gives that $\beta=\frac{1-\alpha}{n}$

$$
x_{i}\left(c^{n}\right)=\left\{\begin{aligned}
\frac{1-\alpha}{n} c_{n}, & \text { if } i<n ; \\
\frac{1-\alpha}{n} c_{n}+\alpha c_{n}, & \text { if } i=k ;
\end{aligned}\right.
$$

Moreover, Independence of Upstream Costs implies that $x_{n}(c)=x_{n}\left(c^{n}\right)=$ $\frac{1-\alpha}{n} c_{n}+\alpha c_{n}$.

By induction on $i$, we assume that $x_{i}(c)=\alpha c_{i}+(1-\alpha) \sum_{j=i}^{n} \frac{1}{j} c_{j}$ for all $i \geq$ $j+1$. Then define $c^{j}$ as $c_{i}^{j}=0$ for $i<j$ and $c_{i}^{j}=c_{i}$ for $i \geq j$. The assumption and Independence of Upstream Costs imply that $x_{i}(c)=x_{i}\left(c^{j}\right)$ for $i \geq j+1$. And with Upstream Strong Surplus Symmetry, we have $x_{i}\left(c^{j}\right)=x_{j}\left(c^{j}\right)-\alpha c_{j}$ for $i<j$. Together with Efficiency, it holds that

$$
\begin{aligned}
x_{j}\left(c^{j}\right) & =\sum_{i=1}^{n} c_{i}-\sum_{i=1}^{j-1} x_{i}\left(c^{j}\right)-\sum_{i=j+1}^{n} x_{i}\left(c^{j}\right) \\
& =\sum_{i=j}^{n} c_{i}-\sum_{i=1}^{j-1}\left[x_{j}\left(c^{j}\right)-\alpha c_{j}\right]-\sum_{i=j+1}^{n}\left[\alpha c_{i}+(1-\alpha) \sum_{j=i}^{n} \frac{1}{j} c_{j}\right]
\end{aligned}
$$

It turns out that $x_{j}\left(c^{j}\right)=\alpha c_{j}+(1-\alpha) \sum_{i=j}^{n} \frac{1}{i} c_{i}$. Again by Independence of Upstream Costs, $x_{j}(c)=x_{j}\left(c^{j}\right)$ is uniquely determined. 



\section{Chapter 5}

\section{The general compromise value for cooperative games}

In this chapter, we introduce a solution concept, the general compromise value, for cooperative games with transferable utility. Different from the existing compromise values in literature, we define the general compromise value with respective to a set of potential payoffs, of which the maximal and minimal potential payoffs are regarded as the upper and lower bound for players. More specifically, the unique pre-imputation on the straight line segment between these two vectors is defined as the general compromise value. By considering different sets of potential payoffs, the corresponding general compromise value coincides with several classical solution concepts, such as the $\tau$-value and the $\chi$-value, which are both well-known compromise solution concepts. Moreover, we provide an axiomatization of the general compromise value based on the relative invariance under $S$-equivalence and the maximal (minimal) proportional property. 


\subsection{Introduction}

The main subject of cooperative game theory [56] is to determine a reasonable and fair allocation rule of the total worth generated by all the participants. Many solution concepts have been introduced to solve this problem. A special type among these well-known solution concepts are called compromise values [84]. For any game, a compromise value selects a unique allocation between two vectors, which are the maximal and minimal payoffs for players. Different ways to measure the maximal and minimal payoffs for players yield various solution concepts. By considering the upper and lower bound of the core [29] as the the maximum and minimum aspiration for players, Tijs [81] proposed the $\tau$-value for transferable utility games. Bergantiños et al. introduced the $\chi$-value for both TU [8] and NTU [9] games by taking into account the maximal marginal contributions for players. Borm et al. [10] extended the $\tau$-value into the NTU-games [5] and proposed two different values, namely the compromise value and the NTU $\tau$-value. A prominent example of a compromise value for bargaining problems [54] is the Raiffa-KalaiSmorodinsky value, which was initially proposed by Raiffa [64] and characterized by Kalai et al [43]. Based on the $\tau$-value for TU and the compromise value for NTU games, Timmer [85] introduced the compromised value for cooperative games with random payoffs.

Notice that in the definition of all existing compromise values except the random cooperative games, the maximal and minimal payoffs for players are based on the game itself, which indicates that once the game is given, the extreme payoff vectors are settled and players have no chance to make any change. But a natural observation is that when facing a decision-making situation, there may exist many restrictions, which can be generated by, for instance, the preferences of players towards the potential payoffs. Recall that, for example, the $\tau$-value is chosen as the unique pre-imputation which lies between the grand marginal contribution vector and the so-called disagreement vector. There is no doubt that it is possible to achieve the upper bound by players. Sometimes, however, there may exist restrictions or preferences that prevent players from achieving their grand marginal contributions. In 
this way, it is no longer reasonable enough to regard the grand marginal contribution vector as an extreme point.

Based on above consideration, we explore the compromise value for cooperative games with transferable utility in a more general way by taking into account the influence of restrictions. Primarily, for any cooperative game, we consider a nonempty bounded subset of the pre-imputation set as the potential payoff vectors for players. Normally, such set is selected from the pre-imputation set, which consists of all payoff vectors that assign the total benefit or cost to all players. We then take advantage of the supremum and infimum payoff for each player among this potential payoffs set to evaluate the maximal and minimal potential payoffs for players. Generally speaking, the total payoff allocated by the maximal potential payoff vector may exceed the worth of the grand coalition, which means that it is impossible to guarantee all players their maximal potential payoffs ("total utopia payoff"). Therefore, all players have to make a concession or compromise towards the amount of the gap between the total utopia payoff and the realistic payoff. On the other hand, the minimal potential payoff vector represents the minimal right for players and generally allocates the total worth of the grand coalition with positive surplus. Similar to the definition of the $\tau$-value, each player is charged a fraction of his maximal contribution to the concession that the grand coalition has to make. In this way, we define the general compromise value as the unique pre-imputation when this proportion is identical for all players. It is worth noting that the general compromise value may lie outside of the set of the potential payoffs. The main reason is that we select the maximal and minimal potential payoff vector respectively as the supremum and infimum point by point. Furthermore, we provide the necessary and sufficient condition when the general compromise value belongs to the core.

In this chapter, we discuss the general compromise value with respect to some special sets of potential payoffs. We first consider the potential payoff set containing pre-imputations of which all players have lower limits towards their final allocation. It turns out that the general compromise value with respect to this kind of sets coincides with several classical solution concepts for cooperative games, including the CIS value [24], the $\alpha$-CIS value [97], the ENSC value [53] and the $\alpha$-ENSC value [80] by setting different lower 
limits for players. When it comes to cost games, players are more likely to set a upper limit towards the sharing of the total cost as their worst results. By choosing various upper limits, the general compromise value also coincides with the mentioned solution concepts in a cost manner. Notice that the general compromise values under these two sets are both included in the set of potential payoffs, but this is not always the case. If we choose the core as the potential payoff set, a counter-example illustrates that the corresponding general compromise value does not belong to the core, but we do provide a necessary and sufficient condition to ensure that. Particularly, once the game is convex, the general compromise value coincides with the $\tau$-value. Recall that the $\tau$-value is the unique pre-imputation on the segment joining the grand marginal contribution vector and the disagreement vector [81]. In this chapter, we consider a payoff set of which the upper and lower bounds for players are exactly the grand marginal contribution vector and the disagreement vector. As a result, the relevant general compromise value is a unique pre-imputation lying on a smaller segment compared with the $\tau$-value. Moreover, we exhibit the sufficient condition when these two values are the same. Another special potential payoff set we take into account consists of pre-imputations with the worth of individual players and the maximal marginal contributions [52] as the lower and upper bound respectively. Interestingly, the corresponding general compromise value is quite similar to the $\chi$-value, proposed by Bergantiños [8], but possesses a different extreme point. We also show the class of games under which the general compromise value coincides with the $\chi$-value.

Furthermore, inspired by the classical characterization of the $\tau$-value [82], we propose an axiomatization to show the fairness and rationality of the general compromise value. It is obvious that the general compromise value is proportional to the maximal (minimal respectively) potential payoff vector whenever the minimal (maximal) potential payoff vector equals zero with respect to a set of potential payoffs. Another prominent property in the axiomatization of the $\tau$-value is the relative invariance under $S$-equivalence, which requires that the value varies in a natural way under the changes in scale which are comparable with positive affine transformations on games. While in our axiomatization of the general compromise value, the positive 
affine transformations affect not only the games but also the set of the potential payoffs. Essentially, the proportional property, the relative invariance under S-equivalence together with the efficiency principle fully characterize the general compromise value on the whole class of cooperative games.

\subsection{Preliminaries}

For any cooperative game $v \in \Gamma_{n}$, the utopia vector $b^{v} \in \mathbf{R}^{n}$ is defined as $b_{i}^{v}:=v(N)-v(N \backslash i), \forall i \in N$, of which the components are the grand marginal contribution of players. The gap function $g^{v}: 2^{N} \rightarrow \mathbf{R}$ is defined as $g^{v}(S):=$ $b^{v}(S)-v(S)=-e^{v}\left(S, b^{v}\right), \forall S \subseteq N$. The concession vector $\lambda^{v} \in \mathbf{R}^{n}$ is given by $\lambda_{i}^{v}:=\min \left\{g^{v}(S) \mid S \subseteq N, i \in S\right\}$. A game $v \in \Gamma_{n}$ is called convex [73] if it holds that

$$
g^{v}(S)+g^{v}(T) \geq g^{v}(S \cup T)+g^{v}(S \cap T) \text { for all } S, T \subseteq N .
$$

Recall that the core is defined as the set of all pre-imputations with nonpositive excesses of all coalitions [29], i.e.,

Definition 5.1. For any cooperative game $v \in \Gamma_{n}$, the core $C(v)$ of game $v$ is given by

$$
C(v):=\left\{x \in \mathbf{R}^{n} \mid x \in I^{*}(v) \text { and } e^{v}(S, x) \leq 0 \text { for all } S \subset N\right\} .
$$

Let $B^{n}$ denote the set of all cooperative games with non-empty core on player set $N$. Tijs [83] revealed that the utopia vector and the disagreement vector are upper and lower bound for the core respectively, i.e.,

Lemma 5.1. Let $v \in B^{n}$ and $x \in C(v)$, then

(i) $x_{i} \leq b_{i}^{v}$ for all $i \in N$ and

(ii) $x_{i} \geq b_{i}^{v}-\lambda_{i}^{v}$ for all $i \in N$.

The $\tau$-value was proposed by Tijs [81] of a quasi-balanced game as the convex combination of the utopia vector $b^{v}$ and the disagreement vector $b^{v}-$ $\lambda^{v}$, i.e., 
Definition 5.2. The $\tau$-value $\tau(v) \in \mathbf{R}^{n}$ of a game $v \in Q B^{n}$ is defined as

$$
\begin{aligned}
\tau(v): & =b^{v}, \quad \text { if } g^{v}(N)=0, \\
& =b^{v}-g^{v}(N)\left(\sum_{j \in N} \lambda_{j}^{v}\right)^{-1} \lambda^{v} \\
& =\left(1-\frac{g^{v}(N)}{\lambda^{v}(N)}\right) b^{v}+\frac{g^{v}(N)}{\lambda^{v}(N)}\left(b^{v}-\lambda^{v}\right) \quad \text { if } g^{v}(N)>0,
\end{aligned}
$$

where $Q B^{n}$ denotes the set of quasi-balanced $n$-person games, which is

$$
Q B^{n}:=\left\{v \in \Gamma_{n} \mid \lambda^{v}(i) \geq 0, \forall i \in N \text { and } 0 \leq g^{v}(N) \leq \lambda^{v}(N)\right\} \text {. }
$$

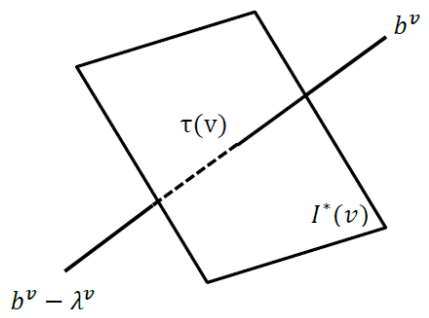

FIGURE 5.1: The $\tau$-value $\tau(v)$ of a quasibalanced game $v$

The idea behind the $\tau$-value as a payoff vector is natural. For any $v \in Q B^{n}$, we have $b^{v}(N) \geq v(N)$, which means that the utopia vector $b^{v}$ generally violates the efficiency principle. So the grand coalition has to make a concession $g^{v}(N)$. Every player $i$ considers the amount $\lambda_{i}^{v}$ as the maximal concession with respect to his utopia payoff $b_{i}^{v}$. Moreover, $\lambda^{v}(N) \geq g^{v}(N)$ implies that each player only need to concede a fraction of his upper bound $\lambda_{i}^{v}$. Once the fractions of all players are the same, the $\tau$-value emerges. In fact, the $\tau$-value is the unique pre-imputation lying on the straight line segment with end points the upper vector $b^{v}$ and the disagreement vector $b^{v}-\lambda^{v}$ (see Figure 5.1), thus the $\tau$-value is always interpreted as a compromise value for cooperative games.

Bergantiños et al. [8] proposed another compromise value, called the $\chi$ value, by modifying the utopia payoff into the maximal marginal contribution [52] for players, that is 
Definition 5.3. For any $v \in I^{n}$, the $\chi$-value $\chi(v)$ is defined as

$$
\chi(v)=m^{\chi}(v)+\alpha\left[M^{\chi}(v)-m^{\chi}(v)\right]
$$

where $I^{n}$ denotes the set of $n$-person games with nonempty imputation set and for all $i \in N$,

$$
\begin{aligned}
& M_{i}^{\chi}(v)=\max _{S \subseteq N, i \in S}\{v(S)-v(S \backslash\{i\})\}, \\
& m_{i}^{\chi}(v)=\max _{S \subseteq N, i \in S}\left\{v(S)-\sum_{j \in S \backslash\{i\}} M_{j}^{\chi}(v)\right\},
\end{aligned}
$$

and $\alpha$ is the real number such that $\sum_{i \in N} \chi_{i}(v)=v(N)$.

Bergantiños [8] has shown that $m_{i}^{\chi}(v)=v(i)$ for all $i \in N$. Note that the $\chi$-value is the unique imputation in the linear segment with the extreme points $m^{\chi}(v)$ and $M^{\chi}(v)$. Inspired by the definition of the $\tau$-value and the $\chi$-value, in this paper, we introduce a more general compromise value by generalizing the minimum and maximum aspirations for players. More details will be shown in the following sections.

\subsection{The general compromise value}

Let $v \in \Gamma_{n}$ be fixed and $\mathscr{F}$ be a nonempty bounded subset of the pre-imputation set $I^{*}(v)$. Here a set $\mathscr{F}$ is bounded in the $n$-dimensional Euclidean space if there exists a real number $r \in(0,+\infty)$ such that $\mathscr{F} \subseteq S(0, r):=\{x \in$ $\left.\mathbf{R}^{n} \mid \sum_{i=1}^{n} x_{i}^{2} \leq r^{2}\right\}$. Essentially, $\mathscr{F}$ can be regarded as a potential payoff set generated from the pre-imputation set with some additional restrictions, which may, for instance, reflect the special preferences of players.

Definition 5.4. Given any $v \in \Gamma_{n}$ and a nonempty bounded subset $\mathscr{F}$ of $I^{*}(v)$, the maximal potential payoff and the minimal potential payoff to players are defined as follows

$$
M_{i}^{\mathscr{F}}(v):=\sup _{x \in \mathscr{F}} x_{i}, \quad \forall i \in N
$$




$$
m_{i}^{\mathscr{F}}(v):=\inf _{x \in \mathscr{F}} x_{i}, \quad \forall i \in N .
$$

Since the subset $\mathscr{F}$ is bounded, the supremum and infimum of $\mathscr{F}$ must exist, hence the definition is valid. Formally, $M^{\mathscr{F}}(v)$ and $m^{\mathscr{F}}(v)$ respectively represent the utopia and minimal payoff for players with respect to the potential payoff set $\mathscr{F}$. Similar to the utopia payoff vector $b^{v}$ and the disagreement vector $b^{v}-\lambda^{v}, M^{\mathscr{F}}(v)$ and $m^{\mathscr{F}}(v)$ generally violate the efficiency principle.

Throughout the chapter, for the sake of simplicity, we denote $\sum_{i \in S} M_{i}^{\mathscr{F}}(v)$ and $\sum_{i \in S} m_{i}^{\mathscr{F}}(v)$ as $M^{\mathscr{F}}(S)$ and $m^{\mathscr{F}}(S)$ for all $S \subseteq N$ respectively. The next lemma exhibits a useful property of the two payoff vector.

Lemma 5.2. Given $v \in \Gamma_{n}, \alpha \in(0,+\infty)$ and $d \in \mathbf{R}^{n}$, let $\mathscr{F}$ be a nonempty bounded subset of $I^{*}(v)$, then it holds that

$$
M^{\mathscr{F}^{\prime}}\left(v^{\prime}\right)=\alpha M^{\mathscr{F}}(v)+d \text { and } m^{\mathscr{F}^{\prime}}\left(v^{\prime}\right)=\alpha m^{\mathscr{F}}(v)+d,
$$

where $\mathscr{F}^{\prime}=\alpha \mathscr{F}+d$ and $v^{\prime}=\alpha v+d$.

The proof is straightforward, thus here we omit it. The following proposition reveals the conditions when $\mathscr{F}$ consists of only one point.

Proposition 5.1. For any $v \in \Gamma_{n}$ and a bounded subset $\mathscr{F}$ of $I^{*}(v)$, the following statements are equivalent.

(i) $\mathscr{F}$ contains only one element;

(ii) $M^{\mathscr{F}}(N)=v(N)$;

(iii) $m^{\mathscr{F}}(N)=v(N)$;

(iv) $M^{\mathscr{F}}(N)=m^{\mathscr{F}}(N)$.

Proof. First, (i) $\Leftrightarrow$ (iv) is straightforward and statement (i) implies both (ii) and (iii). Therefore it is sufficient to prove that (ii) $\Rightarrow$ (i) and (iii) $\Rightarrow$ (i). "(ii) $\Rightarrow$ (i)": Given the condition $M^{\mathscr{F}}(v)(N)=v(N)$, suppose that $\mathscr{F}$ consists of more than one vectors. Let $x, y$ be two different elements in $\mathscr{F}$, then there must exist at least two components $i, j \in N$ such that $x_{i}>y_{i}$ and $x_{j}<y_{j}$. Together with the definition of the maximal potential payoff, it holds that

$$
M_{i}^{\mathscr{F}}(v)=\sup _{z \in \mathscr{F} \subseteq I^{*}(v)} z_{i} \geq x_{i}>y_{i} .
$$


Furthermore we have

$$
M^{\mathscr{F}}(N) \geq y(N \backslash i)+x_{i}>y(N)=v(N) .
$$

The last equation is derived from $y \in I^{*}(v)$. The relation in Equation (5.6) contradicts with the condition $M^{\mathscr{F}}(N)=v(N)$, thus we conclude that $\mathscr{F}$ contains only one element. Similarly, we can prove the case when it comes to the minimal potential payoff.

In the reminder of this chapter, we restrict our attention to the general case, that is, $M^{\mathscr{F}}(N)>v(N)>m^{\mathscr{F}}(N)$. Recall that the maximal potential payoff vector $M^{\mathscr{F}}$ to some degree reflects the ideal payoff when players are facing a choice set $\mathscr{F} . m^{\mathscr{F}}$ indicates the minimal right, which is the baseline for players. Although all players prefer their maximal potential payoff, it is not always possible to distribute the amount $v(N)$ in such a way due to the fact that $M^{\mathscr{F}}(N)>v(N)$. Therefore, the grand coalition has to make a concession. Inspired by the definition of the $\tau$-value, our aim is to find a compromise payoff vector on the segment joining $M^{\mathscr{F}}$ and $m^{\mathscr{F}}$, that is, to select an appropriate parameter $\lambda^{\mathscr{F}}$ and consider the combination $\lambda^{\mathscr{F}} M^{\mathscr{F}}+$ $\left(1-\lambda^{\mathscr{F}}\right) m^{\mathscr{F}}$. Primarily, a natural concern is that the convex combination should satisfy the efficiency principle, i.e.,

$$
\lambda^{\mathscr{F}} M^{\mathscr{F}}(N)+\left(1-\lambda^{\mathscr{F}}\right) m^{\mathscr{F}}(N)=v(N),
$$

which yields that $\lambda^{\mathscr{F}}=\frac{v(N)-m^{\mathscr{F}}(N)}{M^{\mathscr{F}}(N)-m^{\mathscr{F}}(N)}$. It turns out that $\lambda^{\mathscr{F}} \in(0,1)$ because of the assumption that $M^{\mathscr{F}}(N)>v(N)>m^{\mathscr{F}}(N)$. Formally, the following definition is taken into account.

Definition 5.5. For any $v \in \Gamma_{n}$ and nonempty bounded subset $\mathscr{F}$ of $I^{*}(v)$, the general compromise value is defined as

$$
G C^{\mathscr{F}}(v)=\lambda^{\mathscr{F}} M^{\mathscr{F}}(v)+\left(1-\lambda^{\mathscr{F}}\right) m^{\mathscr{F}}(v),
$$

where $\lambda^{\mathscr{F}}=\frac{v(N)-m^{\mathscr{F}}(N)}{M^{\mathscr{F}}(N)-m^{\mathscr{F}}(N)}$. 
It is worth emphasizing that the compromise value may lie outside of the region of $\mathscr{F}$ even if it is a convex combination of the supremum and infimum vector. But we provide necessary and sufficient condition for the compromise value being a core payoff vector.

Theorem 5.3. For any $v \in \Gamma_{n}$ and nonempty bounded subset $\mathscr{F}$ of $I^{*}(v)$, it holds that

$G C^{\mathscr{F}}(v) \in C(v)$ iff $\frac{v(N)-m^{\mathscr{F}}(N)}{M^{\mathscr{F}}(N)-v(N)} \geq \frac{v(S)-m^{\mathscr{F}}(S)}{M^{\mathscr{F}}(S)-v(S)}$, for $S \subseteq N$ satisfying $M^{\mathscr{F}}(S)-v(S)>0$, and $\frac{v(N)-m^{\mathscr{F}}(N)}{M^{\mathscr{F}}(N)-v(N)} \leq \frac{v(S)-m^{\mathscr{F}}(S)}{M^{\mathscr{F}}(S)-v(S)}$, for $S \subseteq N$ satisfying $M^{\mathscr{F}}(S)-v(S)<0$.

Proof. Put $x:=G C^{\mathscr{F}}(v)$, by the formulation (5.8) of the general compromise value, it follows that

$$
\begin{aligned}
& x \in C(v) \text { iff } x(S) \geq v(S), \forall S \subseteq N \\
& \text { iff } \lambda^{\mathscr{F}} M^{\mathscr{F}}(S)+\left(1-\lambda^{\mathscr{F}}\right) m^{\mathscr{F}}(S) \geq v(S), \forall S \subseteq N \\
& \text { iff } \frac{v(N)-m^{\mathscr{F}}(N)}{M^{\mathscr{F}}(N)-m^{\mathscr{F}}(N)} M^{\mathscr{F}}(S)+\frac{M^{\mathscr{F}}(N)-v(N)}{M^{\mathscr{F}}(N)-m^{\mathscr{F}}(N)} m^{\mathscr{F}}(S) \geq v(S), \forall S \subseteq N \\
& \text { iff }\left[M^{\mathscr{F}}(S)-v(S)+v(S)-m^{\mathscr{F}}(S)\right] v(N) \\
& \geq\left[v(S)-m^{\mathscr{F}}(S)\right] M^{\mathscr{F}}(N)+\left[M^{\mathscr{F}}(S)-v(S)\right] m^{\mathscr{F}}(N), \forall S \subseteq N \\
& \text { iff }\left[v(N)-m^{\mathscr{F}}(N)\right]\left[M^{\mathscr{F}}(S)-v(S)\right] \\
& \geq\left[M^{\mathscr{F}}(N)-v(N)\right]\left[v(S)-m^{\mathscr{F}}(S)\right], \forall S \subseteq N .
\end{aligned}
$$

Based on the assumption that $M^{\mathscr{F}}(N)>v(N)>m^{\mathscr{F}}(N)$, we have

$$
x \in C(v) \text { iff } \frac{v(N)-m^{\mathscr{F}}(N)}{M^{\mathscr{F}}(N)-v(N)}\left[M^{\mathscr{F}}(S)-v(S)\right] \geq v(S)-m^{\mathscr{F}}(S), \quad \forall S \subseteq N .
$$

Whenever $M^{\mathscr{F}}(S)-v(S)>0$ or $M^{\mathscr{F}}(S)-v(S)<0$, it follows immediately that Equation (5.9) and Equation (5.10) holds. In case $M^{\mathscr{F}}(S)=v(S)$ for 
some $S \subseteq N$, we have

$$
m^{\mathscr{F}}(S) \leq M^{\mathscr{F}}(S)=v(S)
$$

Hence $v(S)-m^{\mathscr{F}}(S) \leq 0$, which implies that the inequality in (5.11) holds for all $S \subseteq N$ with $M^{\mathscr{F}}(S)=v(S)$.

\subsection{The general compromise value generated by sets with special bounds}

In this section, we further explore the general compromise value generated by some special subsets of the pre-imputation set. Interestingly, different classical solution concepts of the cooperative games can be implemented as the special cases of the general compromise value.

Recall that the main issue in cooperative game theory is to distribute the total worth $v(N)$. Such worth may be benefits or cost produced by all players. When it comes to the benefit case, it is highly possible for players to put a floor under how much they could obtain as their minimal right. Formally, for any $v \in \Gamma_{n}$ and $L \in \mathbf{R}^{n}$, we consider the following subset of $I^{*}(v)$,

$$
\mathscr{F}_{L}=\left\{x \in \mathbf{R}^{n} \mid x(N)=v(N), x_{i} \geq L_{i}, \forall i \in N\right\} .
$$

$L_{i}$ represents the minimal expectation for player $i$ in this game. Clearly, the non-emptiness of $\mathscr{F}_{L}$ is guaranteed by the condition $v(N) \geq L(N)$. In case $v(N)=L(N), \mathscr{F}_{L}$ degenerates into a single point $\{L\}$. Thus we only consider the nontrivial case, that is, $v(N)>L(N)$.

Theorem 5.4. Given any $v \in \Gamma_{n}$ and $\mathscr{F}_{L} \subseteq I^{*}(v)$ satisfying $v(N)>L(N)$, then for all $i \in N$, it holds that

(i) $M_{i}^{\mathscr{F}_{L}}(v)=\sup _{x \in \mathscr{F}_{L}} x_{i}=v(N)-L(N \backslash i)$.

(ii) $m_{i}^{\mathscr{F}_{L}}(v)=\inf _{x \in \mathscr{F}_{L}} x_{i}=L_{i}$.

Proof. (i) Notice that for all $x \in \mathscr{F}_{L}$ and $i \in N$, we have

$$
v(N)-L(N \backslash i) \geq v(N)-x(N \backslash i)=x_{i} .
$$


The above inequality is derived from $x_{i} \geq L_{i}$ and the equality results from the condition $x(N)=v(N)$. It is sufficient to prove that $\forall \varepsilon>0, \exists x \in \mathscr{F}_{L}$ such that $x_{i}>v(N)-L(N \backslash i)-\varepsilon$. To accomplish this, for any fixed $i \in N$ and $\forall \varepsilon>0$, we define the payoff vector $\widehat{x}$ as follows

$$
\widehat{x}_{j}:= \begin{cases}L_{j}+\delta, & \text { if } j \in\{N \backslash i\}, \\ v(N)-L(N \backslash j)-(n-1) \delta, & \text { if } j=i,\end{cases}
$$

where $\delta:=\min \left\{\frac{v(N)-L(N)}{n}, \frac{\varepsilon}{n}\right\}$, which is positive. Obviously, $\widehat{x}(N)=v(N)$ and $\widehat{x}_{j} \geq L_{j}$ for all $j \in\{N \backslash i\}$. As to component $i$, according to the definition of $\delta$, we have

$$
\widehat{x}_{i} \geq v(N)-L(N \backslash i)-\frac{n-1}{n}(v(N)-L(N))=\frac{1}{n}(v(N)-L(N))+L_{i}>L_{i} .
$$

The above discussion gives that $\hat{x} \in \mathscr{F}_{L}$. Moreover, also based on the definition of $\delta$, it yields

$$
\widehat{x}_{i}-(v(N)-L(N \backslash i)-\varepsilon)=\varepsilon-(n-1) \delta \geq \varepsilon-\frac{n-1}{n} \varepsilon=\frac{1}{n} \varepsilon>0 .
$$

Thus we have $\widehat{x}_{i}>v(N)-L(N \backslash i)-\varepsilon$, which completes the proof of (i).

(ii) Obviously, it is sufficient to prove that $\forall \varepsilon>0, \exists x \in \mathscr{F}_{L}$ such that $x_{i}<L(i)+\varepsilon$. For any $i \in N$ and $\forall \varepsilon>0$, the vector $\widehat{x} \in \mathbf{R}^{n}$ as

$$
\widehat{x}_{j}:= \begin{cases}L_{j}+\frac{v(N)-L(N)}{n-1}-\delta, & \text { if } j \in\{N \backslash i\}, \\ L_{j}+(n-1) \delta, & \text { if } j=i,\end{cases}
$$

where $\delta:=\min \left\{\frac{v(N)-L(N)}{n}, \frac{\varepsilon}{n}\right\}>0$. Similarly to the proof of (i), one may easily verify that $\widehat{x} \in \mathscr{F}_{L}$, and $\forall \varepsilon>0$, it holds that $\widehat{x}_{i}<L(i)+\varepsilon$.

Based on Theorem 5.4, we obtain the parameter $\lambda^{\mathscr{F}_{L}}$ as

$$
\lambda^{\mathscr{F}_{L}}=\frac{v(N)-m^{\mathscr{F}_{L}}(N)}{M^{\mathscr{F}_{L}}(N)-m^{\mathscr{F}_{L}}(N)}=\frac{v(N)-L(N)}{n v(N)-\sum_{j \in N} L(N \backslash j)-L(N)}=\frac{1}{n}
$$


It follows that the general compromise value in this case has the form

$$
\begin{aligned}
G C_{i}^{\mathscr{F}_{L}}(v) & =\lambda^{\mathscr{F}_{L}} M_{i}^{\mathscr{F}_{L}}(v)+\left(1-\lambda^{\mathscr{F}_{L}}\right) m_{i}^{\mathscr{F}_{L}}(v) \\
& =\frac{1}{n}(v(N)-L(N \backslash i))+\left(1-\frac{1}{n}\right) L_{i} \\
& =L_{i}+\frac{v(N)-L(N)}{n}, \quad \forall i \in N .
\end{aligned}
$$

Remark 5.1. Various classical solution concepts for cooperative games can be obtained via different choices of the vector $L$.

Case 1: Let $L:=\{v(i)\}_{i \in N}$, then the general compromise value coincides with the CIS value [24], i.e.,

$$
G C_{i}^{\mathscr{F}_{L}}(v)=v(i)+\frac{v(N)-\sum_{j \in N} v(j)}{n} \text { with } v(N)>\sum_{j \in N} v(j) .
$$

Case 2: Let $L:=\left\{\alpha_{i} v(i)\right\}_{i \in N}$, where $\alpha_{i} \in[0,1]$. Then the general compromise value coincides with the $\alpha$-CIS value [97], i.e.,

$$
G C_{i}^{\mathscr{F}_{L}}(v)=\alpha_{i} v(i)+\frac{v(N)-\sum_{j \in N} \alpha_{j} v(j)}{n} \text { with } v(N)>\sum_{j \in N} \alpha_{j} v(j) .
$$

Case 3: Let $L:=\left\{b_{i}^{v}\right\}_{i \in N}$, it follows that the general compromise value becomes the ENSC value [53], that is,

$$
G C_{i}^{\mathscr{F}_{L}}(v)=b_{i}^{v}+\frac{v(N)-\sum_{j \in N} b_{j}^{v}}{n} \text { with } v(N)>\sum_{j \in N} b_{i}^{v} .
$$

Case 4: Let $L:=\left\{\alpha_{i} b_{i}^{v}\right\}_{i \in N}$, where $\alpha_{i} \in[0,1]$. It turns out that the general compromise value coincides with the $\alpha$-ENSC value [80], that is,

$$
G C_{i}^{\mathscr{F}_{L}}(v)=\alpha_{i} b_{i}^{v}+\frac{v(N)-\sum_{j \in N} \alpha_{j} b_{j}^{v}}{n} \text { with } v(N)>\sum_{j \in N} \alpha_{j} b_{i}^{v} .
$$

Once the worth $v(N)$ refers to cost, for instance, in the Tennessee Valley 
Authority (TVA) cost allocation problem [65], players certainly prefer to obtain as few distribution as possible. But due to the restriction of efficiency principle, players may at best set a cap of the cost sharing as their worst outcomes. Thus for any $v \in \Gamma_{n}$ and $R \in \mathbf{R}^{n}$, we consider the subset of the pre-imputation set given by

$$
\mathscr{F}_{R}=\left\{x \in \mathbf{R}^{n} \mid x(N)=v(N), x_{i} \leq R_{i}, \forall i \in N\right\} .
$$

$R$ can be regarded as the maximal share of the total cost. $\mathscr{F}_{R}$ is nonempty if and only if $v(N) \leq R(N)$, and $\mathscr{F}_{R}$ degenerates into a one point when it holds that $v(N)=R(N)$. The next theorem gives the supremum and infimum of the set $\mathscr{F}_{R}$.

Theorem 5.5. Given any $v \in \Gamma_{n}$ and $\mathscr{F}_{R} \subseteq I^{*}(v)$ satisfying $v(N)<R(N)$, then for all $i \in N$, it holds that

(i) $M_{i}^{\mathscr{F}_{R}}(v)=\sup _{x \in \mathscr{F}_{R}} x_{i}=R_{i}$.

(ii) $m_{i}^{\mathscr{F}_{R}}(v)=\inf _{x \in \mathscr{F}_{R}} x_{i}=v(N)-R(N \backslash i)$.

Proof. (i) Obviously, for any $i \in N$ and $x \in \mathscr{F}_{R}, x_{i} \leq R_{i} . \forall \varepsilon>0$, it remains to prove that there must exist vector $x \in \mathscr{F}_{R}$ such that $x_{i}>R_{i}-\varepsilon$. Given any $\varepsilon>0$, we define the vector $\widehat{x}$ as follows

$$
\widehat{x}_{j}:= \begin{cases}R_{j}+\frac{v(N)-R(N)}{n-1}+\delta, & \text { if } j \in\{N \backslash i\}, \\ R_{j}-(n-1) \delta, & \text { if } j=i,\end{cases}
$$

where $\delta:=\min \left\{\frac{R(N)-v(N)}{n}, \frac{\varepsilon}{n}\right\}>0$. It is not difficult to verify that $\widehat{x} \in \mathscr{F}_{R}$. Further, $\forall \varepsilon>0$, it follows that

$$
\widehat{x}_{i}=R_{j}-(n-1) \delta>R_{j}-\frac{n-1}{n} \varepsilon>R_{i}-\varepsilon,
$$

hence $R_{i}$ is exactly the supremum payoff of player $i$. Note that the proof of (ii) is quite similar to (i), here we omit it. 
The determination of the maximal and minimal potential payoff yields that the coefficient is

$$
\lambda^{\mathscr{F}_{R}}=\frac{v(N)-\left(n v(N)-\sum_{j \in N} R(N \backslash j)\right)}{R(N)-\left(n v(N)-\sum_{j \in N} R(N \backslash j)\right)}=\frac{n-1}{n} .
$$

By the definition of the general compromise value, we have

$$
\begin{aligned}
G C_{i}^{\mathscr{F}_{R}}(v) & =\lambda^{\mathscr{F}_{R}} M_{i}^{\mathscr{F}_{R}}(v)+\left(1-\lambda^{\mathscr{F}_{R}}\right) m_{i}^{\mathscr{F}_{R}}(v) \\
& =\frac{n-1}{n} R_{i}+\left(1-\frac{n-1}{n}\right)(v(N)-R(N \backslash i)) \\
& =R_{i}+\frac{v(N)-R(N)}{n}, \quad \forall i \in N .
\end{aligned}
$$

Remark 5.2. Similarly, different classical solution concepts for cooperative games can be implemented when choosing different vector $R$.

Case 1: Let $R:=\{v(i)\}_{i \in N}$, then the general compromise value coincides with the CIS value, i.e.,

$$
G C_{i}^{\mathscr{F}_{R}}(v)=v(i)+\frac{v(N)-\sum_{j \in N} v(j)}{n} \text { with } v(N)<\sum_{j \in N} v(j) .
$$

Case 2: Let $R:=\left\{\alpha_{i} v(i)\right\}_{i \in N}$, where $\alpha_{i} \in[0,1]$. Then the general compromise value coincides with the $\alpha$-CIS value, i.e.,

$$
G C_{i}^{\mathscr{F}_{R}}(v)=\alpha_{i} v(i)+\frac{v(N)-\sum_{j \in N} \alpha_{j} v(j)}{n} \text { with } v(N)<\sum_{j \in N} \alpha_{j} v(j) .
$$

Case 3: Let $R:=\left\{b_{i}^{v}\right\}_{i \in N}$, it follows that the general compromise value becomes the ENSC value, that is,

$$
G C_{i}^{\mathscr{F}_{R}}(v)=b_{i}^{v}+\frac{v(N)-\sum_{j \in N} b_{j}^{v}}{n} \text { with } v(N)<\sum_{j \in N} b_{i}^{v} .
$$


Case 4: Let $R:=\left\{\alpha_{i} b_{i}^{v}\right\}_{i \in N}$, where $\alpha_{i} \in[0,1]$. It turns out that the general compromise value coincides with the $\alpha$-ENSC value, that is,

$$
G C_{i}^{\mathscr{F}_{R}}(v)=\alpha_{i} b_{i}^{v}+\frac{v(N)-\sum_{j \in N} \alpha_{j} b_{j}^{v}}{n} \text { with } v(N)<\sum_{j \in N} \alpha_{j} b_{i}^{v} .
$$

Generally speaking, it is not realistic for players to claim their distribution of benefits or cost without limits. We now consider a more restrictive situation, that is, there exists both upper and lower boundary of the payoff for players. For any $v \in \Gamma_{n}$ and $L, R \in \mathbf{R}^{n}$, let $\mathscr{F}_{T}$ be a subset of $I^{*}(v)$ given by

$$
\mathscr{F}_{T}=\left\{x \in \mathbf{R}^{n} \mid x(N)=v(N), L_{i} \leq x_{i} \leq R_{i}, \forall i \in N\right\}
$$

Note that $\mathscr{F}_{T}$ is nonempty if and only if $L(N) \leq v(N) \leq R(N)$ and $L_{i} \leq R_{i}$ for all $i \in N$.

Proposition 5.2. For any $v \in \Gamma_{n}$ and nonempty subset $\mathscr{F}_{T}$ of $I^{*}(v)$, if

(i) $L(N)=v(N)$ or

(ii) $R(N)=v(N)$ or

(iii) $L(N)=R(N)$,

then $\mathscr{F}_{T}$ contains only one element.

In view of Proposition 5.2, we leave out the scenario in which $\mathscr{F}_{T}$ degenerates into a single point, and focus on the nontrivial case, that is, $L(N)<$ $v(N)<R(N)$.

Theorem 5.6. Let $v \in \Gamma_{n}$ and $\mathscr{F}_{T}$ be a nonempty subset of $I^{*}(v)$ with $L(N)<$ $v(N)<R(N)$. The maximal potential payoff and minimal potential payoff for any $i \in N$ are given as

(i) $M_{i}^{\mathscr{F}_{T}}(v)=\sup _{x \in \mathscr{F}_{T}} x_{i}=\min \left\{v(N)-L(N \backslash i), R_{i}\right\}$.

(ii) $m_{i}^{\mathscr{F}_{T}}(v)=\inf _{x \in \mathscr{F}_{T}} x_{i}=\max \left\{L_{i}, v(N)-R(N \backslash i)\right\}$.

Proof. Primarily, we show that for all $x \in \mathscr{F}_{T}$, it follows that $x_{i} \leq \min \{v(N)-$ $\left.L(N \backslash i), R_{i}\right\}$. Suppose that there exists $\tilde{x} \in \mathscr{F}_{T}$ such that $\tilde{x}_{i}>\min \{v(N)-$ $\left.L(N \backslash i), R_{i}\right\}$, next we analyze the two cases respectively.

Case 1: If $\min \left\{v(N)-L(N \backslash i), R_{i}\right\}=R_{i}$, then it follows that $\widetilde{x}_{i}>R_{i}$, which 
contradicts with the condition $\widetilde{x}_{i} \leq R_{i}$

Case 2: If $\min \left\{v(N)-L(N \backslash i), R_{i}\right\}=v(N)-L(N \backslash i)$, then we have $\tilde{x}_{i}>v(N)-$ $L(N \backslash i)$, which implies that

$$
v(N)-\widetilde{x}_{i}=\tilde{x}(N \backslash i)<L(N \backslash i) .
$$

The equation is derived from that $\widetilde{x}(N)=v(N)$. Recall that $\widetilde{x}_{j}>L_{j}$ for all $j \in N$, thus it holds that $\tilde{x}(N \backslash i)>L(N \backslash i)$, which contradicts with equation (5.18).

Based on above discussion, we conclude that $x_{i} \leq \min \left\{v(N)-L(N \backslash i), R_{i}\right\}$ for all $x \in \mathscr{F}_{T}$. It remains to prove that for a fixed $i \in N$ and $\forall \varepsilon>0$, there exists $\widehat{x} \in \mathscr{F}_{T}$ satisfying $\widehat{x}_{i}>\min \left\{v(N)-L(N \backslash i), R_{i}\right\}-\varepsilon$. Similarly, we construct the payoff vector $\widehat{x}$ by considering different cases.

Case 1: If $\min \left\{v(N)-L(N \backslash i), R_{i}\right\}=R_{i}$, define the vector $\widehat{x}$ as

$$
\widehat{x}_{j}:= \begin{cases}\delta L_{j}+(1-\delta) R_{j}, & \text { if } j \in\{N \backslash i\}, \\ v(N)-\delta L(N \backslash i)-(1-\delta) R(N \backslash i), & \text { if } j=i,\end{cases}
$$

where

$$
\delta:= \begin{cases}\frac{1}{2} \frac{R(N)-v(N)-\varepsilon}{R(N \backslash i)-L(N \backslash i)}+\frac{1}{2}, & \text { if } R(N)-v(N)-\varepsilon>0, \\ \frac{1}{2}, & \text { if } R(N)-v(N)-\varepsilon \leq 0 .\end{cases}
$$

In case $R(N \backslash i)-L(N \backslash i)=0$, then $\mathscr{F}_{T}$ degenerates into a single point $x$ of which $x_{j}=L_{j}=R_{j}$ for all $j \in\{N \backslash i\}$ and $x_{i}=v(N)-L(N \backslash i)=R(N \backslash i)$. Thus we focus on the case where $R(N \backslash i)-L(N \backslash i)>0$. Moreover, it holds that

$$
\begin{aligned}
\frac{R(N)-v(N)-\varepsilon}{R(N \backslash i)-L(N \backslash i)} & =\frac{R(N \backslash i)-\left(v(N)-R_{i}\right)-\varepsilon}{R(N \backslash i)-L(N \backslash i)} \\
& \leq \frac{R(N \backslash i)-L(N \backslash i)-\varepsilon}{R(N \backslash i)-L(N \backslash i)} \\
& =1-\frac{\varepsilon}{R(N \backslash i)-L(N \backslash i)}<1 .
\end{aligned}
$$

The last but one inequality is derived from that $v(N)-L(N \backslash i) \geq R_{i}$. Hence it follows that $\delta \in(0,1)$, which implies $L_{k} \leq \widehat{x} \leq R_{k}$ for all $k \in N$. Together with the efficiency of $\widehat{x}$, we conclude that $\widehat{x} \in \mathscr{F}_{T}$. Next we show that $\widehat{x}_{i}>$ 
$R_{i}-\varepsilon$.

When $R(N)-v(N)-\varepsilon>0$, then

$$
\begin{aligned}
\widehat{x}_{i} & =v(N)-R(N \backslash i)-\frac{1}{2}\left[\frac{R(N)-v(N)-\varepsilon}{R(N \backslash i)-L(N \backslash i)}+1\right][L(N \backslash i)-R(N \backslash i)] \\
& >v(N)-R(N \backslash i)-\frac{R(N)-v(N)-\varepsilon}{R(N \backslash i)-L(N \backslash i)}[L(N \backslash i)-R(N \backslash i)] \\
& =R_{i}-\varepsilon .
\end{aligned}
$$

When $R(N)-v(N)-\varepsilon \leq 0$, we have

$$
\begin{aligned}
\widehat{x}_{i} & =v(N)-R(N \backslash i)-\frac{1}{2}[L(N \backslash i)-R(N \backslash i)] \\
& =R_{i}+v(N)-R(N)+\frac{1}{2}[R(N \backslash i)-L(N \backslash i)] \\
& =R_{i}-\varepsilon .
\end{aligned}
$$

Case 2: If $\min \left\{v(N)-L(N \backslash i), R_{i}\right\}=v(N)-L(N \backslash i)$, similar to case 1, define $\widehat{x}$ as

$$
\widehat{x}_{j}:= \begin{cases}\delta L_{j}+(1-\delta) R_{j}, & \text { if } j \in\{N \backslash i\}, \\ v(N)-\delta L(N \backslash i)-(1-\delta) R(N \backslash i), & \text { if } j=i,\end{cases}
$$

where

$$
\delta:= \begin{cases}1-\frac{1}{2} \frac{\varepsilon}{R(N \backslash i)-L(N \backslash i)}, & \text { if } R(N \backslash i)-L(N \backslash i)-\varepsilon>0, \\ \frac{1}{2}, & \text { if } R(N \backslash i)-L(N \backslash i)-\varepsilon \leq 0 .\end{cases}
$$

One could verify that $\delta \in(0,1)$, which implies that $\widehat{x} \in \mathscr{F}_{T}$. Furthermore, we exhibit that $\widehat{x}_{i}>v(N)-L(N \backslash i)-\varepsilon$.

When $R(N \backslash i)-L(N \backslash i)-\varepsilon>0$, then

$$
\begin{aligned}
\widehat{x}_{i} & =v(N)-R(N \backslash i)-\left[1-\frac{1}{2} \frac{\varepsilon}{R(N \backslash i)-L(N \backslash i)}\right][L(N \backslash i)-R(N \backslash i)] \\
& >v(N)-R(N \backslash i)-\left[1-\frac{\varepsilon}{R(N \backslash i)-L(N \backslash i)}\right][L(N \backslash i)-R(N \backslash i)] \\
& =v(N)-L(N \backslash i)-\varepsilon .
\end{aligned}
$$


When $R(N \backslash i)-L(N \backslash i)-\varepsilon \leq 0$, we have

$$
\begin{aligned}
\widehat{x}_{i} & =v(N)-R(N \backslash i)-\frac{1}{2}[L(N \backslash i)-R(N \backslash i)] \\
& =v(N)-L(N \backslash i)-\frac{1}{2}[R(N \backslash i)-L(N \backslash i)] \\
& \geq R_{i}-\frac{1}{2} \varepsilon>R_{i}-\varepsilon,
\end{aligned}
$$

Which completes the proof of (i). The proof of (ii) is left to the reader, since the method is the same as (i).

Now that we have figured out the maximal and minimal potential payoff for players with respect to the set $\mathscr{F}_{T}$, the corresponding general compromise value $G C^{\mathscr{F}_{T}}$ can be acquired. Furthermore, it turns out that $M_{i}^{\mathscr{F}_{T}}(v) \leq R_{i}$ and $m_{i}^{\mathscr{F}_{T}}(v) \geq L_{i}$ for all $i \in N$, which implies that $G C^{\mathscr{F}_{T}} \in \mathscr{F}_{T}$. The same result holds by $G C^{\mathscr{F}_{L}}$ and $G C^{\mathscr{F}_{R}}$, that is, $G C^{\mathscr{F}_{L}} \in \mathscr{F}_{L}$ and $G C^{\mathscr{F}_{R}} \in \mathscr{F}_{R}$. But it is not always true that $G C^{\mathscr{F}} \in \mathscr{F}$, and some counter-examples will be shown later.

\subsection{The general compromise value, the $\tau$-value, the $\chi$-value and the core}

In the theory of cooperative games, the core is a rather significant solution concept. The core is always internally stable, since all coalitions can receive at least the worth by acting alone and have no incentive to drop out the sharing system. But there still exists one defect, that is, the core of a game generally is not a single point, which is clearly not an ideal choice in decision issues. In this section, we explore the general compromise value generated from the core set, and study the relationship of the general compromise value with the $\tau$-value, the $\chi$-value and the core.

\subsubsection{The general compromise value generated from the core}

For any cooperative game $v$ with a non-empty core, i.e., $v \in B^{n}$, let $\mathscr{F}_{C}:=$ $C(v)$. Since $\mathscr{F}_{C}$ is a nonempty compact set, the maximal and minimal potential payoff vector $M^{\mathscr{F}_{C}}(v)$ and $m^{\mathscr{F}_{C}}(v)$ are both well-defined. Therefore, 
we define the general compromise value $G C^{\mathscr{F}_{C}}(v)$ generated from the core. In general, it does not always hold that $G C^{\mathscr{F}} C(v) \in C(v)$, which can be illustrated by the following example.

Example 5.1. Let $v \in \Gamma_{n}$ be a 5-person game, and the characteristic function is given as

$$
v(S):= \begin{cases}0, & \text { if }|S|=1, \\ 8, & \text { if }|S|=2 \text { and } 1 \in S, \\ 10, & \text { if }|S|=2,1 \notin S \text { and } S \neq\{4,5\}, \\ 36, & \text { if } S=\{4,5\}, \\ 18, & \text { if }|S|=3,1 \in S \text { and } S \neq\{1,4,5\}, \\ 44, & \text { if } S=\{1,4,5\}, \\ 20, & \text { if }|S|=3,1 \notin S, \\ 28, & \text { if }|S|=4,\{4,5\} \nsubseteq S, \\ 54, & \text { if }|S|=4,\{2,3\} \nsubseteq S, \\ 56, & \text { if } S=\{2,3,4,5\}, \\ 64, & \text { if } S=N .\end{cases}
$$

Essentially, the game is convex, of which the two vectors $b^{v}$ and $b^{v}-\lambda^{v}$ are sharp bounds for the core [81]. Thus we have $M^{\mathscr{F}_{C}}(v)=b^{v}=(8,10,10,36,36)$ and $m^{\mathscr{F}_{C}}(v)=b^{v}-\lambda^{v}=(0,0,0,0)$, which gives that $\lambda^{\mathscr{F}_{C}}=\frac{v(N)-m^{\mathscr{F} C}(N)}{M^{\mathscr{F} C}(N)-m^{\mathscr{F} C}(N)}=$ 0.64 and $G C^{\mathscr{F}} C(v)=0.64(8,10,10,36,36)$. Observe that $G C^{\mathscr{F}_{C}}(\{123\})=$ $0.64(8+10+10)=17.92<18=v(\{123\})$, which contradicts with the definition of the core. Hence we conclude that $G C^{\mathscr{F}_{C}} \notin C(v)$.

As a matter of fact, once the game is convex, the two vectors $b^{v}$ and $b^{v}-\lambda^{v}$ are sharp bounds for the core, that is

$$
M^{\mathscr{F}_{C}}(v)=b^{v} \text { and } m^{\mathscr{F}_{C}}(v)=b^{v}-\lambda^{v}
$$

Therefore, the compromise value $G C^{\mathscr{F}_{C}}(v)$ coincides with the $\tau$-value for any convex game [81]. The next corollary shows the necessary and sufficient conditions when the general compromise value belongs to the core. 
Corollary 5.1. For any $v \in B^{n}$, let $\mathscr{F}_{C}:=C(v)$, then it holds that $G C^{\mathscr{F}_{C}}(v) \in \mathscr{F}_{C}$ iff $\frac{v(N)-m^{\mathscr{F}_{C}}(N)}{M^{\mathscr{F}}(N)-v(N)} \geq \frac{v(S)-m^{\mathscr{F}_{C}}(S)}{M^{\mathscr{F}_{C}}(S)-v(S)}$, for $S \subseteq N$ satisfying $M^{\mathscr{F}_{C}}(S)-v(S)>0$.

Notice that Corollary 5.1 is a direct variation of Theorem 5.3. By the definition of the maximal potential payoff, we have $M^{\mathscr{F}_{C}}(S) \geq v(S)$ for all $S \subseteq N$ when $\mathscr{F}_{C}=C(v)$. So we only need to take into account the case $M^{\mathscr{F}_{C}}(S)-v(S)>0$.

\subsubsection{The relation between the general compromise value and the $\tau$-value}

Recall that for any $v \in B^{n}$, the vectors $b^{v}$ and $b^{v}-\lambda^{v}$ respectively are upper and lower bound for the core of the game $v$. Our aim is to study the general compromise value by considering the two bounds. For that purpose, given any $v \in \Gamma_{n}$, we consider the subset $\mathscr{F}_{\tau}$ of $I^{*}(v)$ with $L:=b^{v}-\lambda^{v}$ and $R:=b^{v}$, i.e.,

$$
\mathscr{F}_{\tau}=\left\{x \in \mathbf{R}^{n} \mid x(N)=v(N), b_{i}^{v}-\lambda_{i}^{v} \leq x_{i} \leq b_{i}^{v}, \forall i \in N\right\}
$$

Actually, the set $\mathscr{F}_{\tau}$ in this type is nonempty if and only if the games are quasi-balanced, i.e.,

Proposition 5.3. Given $v \in \Gamma_{n}$ and $\mathscr{F}_{\tau}=\left\{x \in \mathbf{R}^{n} \mid x(N)=v(N), b_{i}^{v}-\lambda_{i}^{v} \leq\right.$ $\left.x_{i} \leq b_{i}^{v}, \forall i \in N\right\}$, then $\mathscr{F}_{\tau} \neq \emptyset$ if and only if $v \in Q B^{n}$.

Proof.

$\mathscr{F}_{\tau} \neq \emptyset$ iff $\quad b_{i}^{v}-\lambda_{i}^{v} \leq b_{i}^{v}$ for all $i \in N$ and $b^{v}(N)-\lambda^{v}(N) \leq v(N) \leq b^{v}(N)$

iff $\quad \lambda_{i}^{v} \geq 0$, for all $i \in N$ and $b^{v}(N)-\lambda^{v}(N) \leq v(N) \leq b^{v}(N)$. 
Observe that conditions in Equation (5.26) are equivalent to the definition of quasi-balanced games as shown in Equation (5.2).

According to Theorem 5.6, we could immediately obtain the maximal and minimal potential payoff vector of this particular subset.

Corollary 5.2. For any $v \in Q B^{n}$ and $\mathscr{F}_{\tau}=\left\{x \in \mathbf{R}^{n} \mid x(N)=v(N), b_{i}^{v}-\lambda_{i}^{v} \leq\right.$ $\left.x_{i} \leq b_{i}^{v}, \forall i \in N\right\}$, the maximal potential payoff and minimal potential payoff for any $i \in N$ are given as

(i) $M_{i}^{\mathscr{F}_{\tau}}(v)=\min \left\{v(N)-\left[b^{v}(N \backslash i)-\lambda^{v}(N \backslash i)\right], b_{i}^{v}\right\}$.

(ii) $m_{i}^{\mathscr{F}_{\tau}}(v)=\max \left\{b_{i}^{v}-\lambda_{i}^{v}, v(N)-b^{v}(N \backslash i)\right\}$.

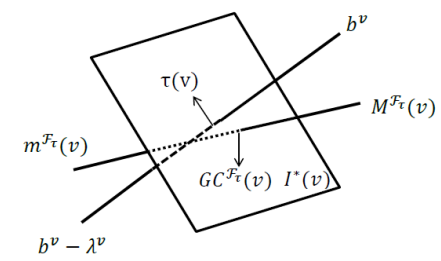

FIGURE 5.2: The general compromise value $G C^{\mathscr{F}_{\tau}}(v)$ of a quasibalanced game $v$.

Notice that $b_{i}^{v} \geq M_{i}^{\mathscr{F}_{\tau}}(v)$ and $b_{i}^{v}-\lambda_{i}^{v} \leq m_{i}^{\mathscr{F}_{\tau}}(v)$, which implies that the general compromise value in this case is selected from a smaller range compared to the $\tau$-value. Figure 5.2 illustrates this relationship. Furthermore, the next theorem shows that the compromise value coincides with the $\tau$-value under certain subclass games, which are included in the set of all quasibalanced games.

Theorem 5.7. For any $v \in S Q B^{n}:=\left\{v \in \Gamma_{n} \mid 0 \leq \lambda_{i}^{v} \leq g^{v}(N) \leq \lambda^{v}(N \backslash i), \forall i \in\right.$ $N\}$ and $\mathscr{F}_{\tau}=\left\{x \in \mathbf{R}^{n} \mid x(N)=v(N), b_{i}^{v}-\lambda_{i}^{v} \leq x_{i} \leq b_{i}^{v}, \forall i \in N\right\}$, the general compromise value coincides with the $\tau$-value.

Proof. Let $v \in S Q B^{n}$ and $i \in N$, then we have

$$
b^{v}(N)-\lambda^{v}(N) \leq b^{v}(N)-\lambda^{v}(N \backslash i) \leq v(N) \leq b^{v}(N)-\lambda_{i}^{v} \leq b^{v}(N) .
$$

The first and last inequalities hold because of the fact that $\lambda_{i}^{v} \geq 0 . g^{v}(N)=$ $b^{v}(N)-v(N) \leq \lambda^{v}(N \backslash i)$ yields that the second inequality, and $\leq b^{v}(N)-\lambda_{i}^{v}$ 
is derived from the condition $\lambda_{i}^{v} \leq g^{v}(N)=b^{v}(N)-v(N)$. Equation (5.27) implies that $v \in Q B^{n}$, thus by Corollary 5.2, it follows that

$$
\begin{aligned}
& M_{i}^{\mathscr{F}_{\tau}}(v)=\min \left\{v(N)-\left[b^{v}(N \backslash i)-\lambda^{v}(N \backslash i)\right], b_{i}^{v}\right\}, \\
& m_{i}^{\mathscr{F}_{\tau}}(v)=\max \left\{b_{i}^{v}-\lambda_{i}^{v}, v(N)-b^{v}(N \backslash i)\right\} .
\end{aligned}
$$

To prove the coincidence of the general compromise value and the $\tau$-value, it is sufficient to show that

$$
M_{i}^{\mathscr{F}_{\tau}}(v)=b_{i}^{v}, \quad m_{i}^{\mathscr{F}_{\tau}}(v)=b_{i}^{v}-\lambda_{i}^{v},
$$

which are equivalent to

$$
\begin{aligned}
& b_{i}^{v} \leq v(N)-\left[b^{v}(N \backslash i)-\lambda^{v}(N \backslash i)\right], \quad b_{i}^{v}-\lambda_{i}^{v} \geq v(N)-b^{v}(N \backslash i) \\
& \Leftrightarrow b^{v}(N)-v(N) \leq \lambda^{v}(N \backslash i), \quad b^{v}(N)-v(N) \geq \lambda_{i}^{v} \\
& \Leftrightarrow \lambda_{i}^{v} \leq b^{v}(N)-v(N) \leq \lambda^{v}(N \backslash i) \\
& \Leftrightarrow \lambda_{i}^{v} \leq g^{v}(N) \leq \lambda^{v}(N \backslash i) .
\end{aligned}
$$

Apparently, equation (5.28) holds since $v \in S Q B^{n}$.

Remark 5.3. Driessen [21] showed that the $\tau$-value and the nucleolus both coincide with the barycenter of the core on the domain of 1-convex games, which is given by

$$
C_{1}^{n}=\left\{v \in \Gamma_{n} \mid 0 \leq g^{v}(N) \leq g^{v}(S), \text { for all } S \subset N\right\} .
$$

It is not difficult to verify that $C_{1}^{n} \subseteq S Q B^{n}$, therefore we conclude that the general compromise value and the nucleolus coincide with the barycenter of the core for any 1-convex game. When it comes to a smaller subclass of games, i.e., convex games, it holds that the general compromise value, the nucleolus and the Shapley value [72] are all the barycenter of the core, which is a resemblance with the result of [73]. 


\subsubsection{The relation between the general compromise value and the $\chi$-value}

The concept of maximal marginal contribution was initially introduced by Milnor [52], and van Heumen [38] took advantage of the concept to define the so-called $\mu$-value. To explore the general compromise value with such special bounds, for any $v \in \Gamma_{n}$, we formally consider the following subsets of the pre-imputation set, that is,

$$
\mathscr{F}_{\chi}=\left\{x \in \mathbf{R}^{n} \mid x(N)=v(N), m_{i}^{\chi}(v) \leq x_{i} \leq M_{i}^{\chi}(v), \forall i \in N\right\} .
$$

Proposition 5.4. Given $v \in \Gamma_{n}$ and $\mathscr{F}_{\chi}=\left\{x \in \mathbf{R}^{n} \mid x(N)=v(N), m_{i}^{\chi}(v) \leq\right.$ $\left.x_{i} \leq M_{i}^{\chi}(v), \forall i \in N\right\}$, then $\mathscr{F}_{\chi} \neq \emptyset$ if and only if $v \in I^{n}$.

Proof.

$$
\begin{array}{rlrl}
\mathscr{F}_{\chi} \neq \emptyset \text { iff } & v(\{i\}) & \leq \max _{S \ni i}\{v(S)-v(S \backslash i)\} \text { for all } i \in N \\
\text { and } \quad \sum_{i \in N} v(\{i\}) & \leq v(N) \leq \sum_{i \in N} \max _{S \ni i}\{v(S)-v(S \backslash i)\} .
\end{array}
$$

It is easy to see Equation (5.31) holds by taking $S=\{i\}$. To prove $v(N) \leq$ $\sum_{i \in N} \max _{S \ni i}\{v(S)-v(S \backslash i)\}$, let $\theta$ be a permutation of $N$ and $p_{i}^{\theta}$ be the set containing players who precede player $i$ under the ordering $\theta$. Then it follows that

$$
v(N)=\sum_{j \in N}\left\{v\left(p_{j}^{\theta} \cup\{j\}\right)-v\left(p_{j}^{\theta}\right)\right\} \leq \sum_{j \in N} \max _{S \ni j}\{v(S)-v(S \backslash j)\} .
$$

Furthermore, $\sum_{i \in N} v(\{i\}) \leq v(N)$ holds if and only if the imputation set $I(v)$ is nonempty, that is, $v \in I^{n}$.

Notably, if we choose the minimal marginal contributions for players as the lower bound, i.e., $m_{i}^{\chi}(v):=\min _{S \ni i}\{v(S)-v(S \backslash i)\}$, it is not difficult to verify that $\mathscr{F}_{\chi} \neq \emptyset$ for any $v \in \Gamma_{n}$. The next corollary states that the minimal potential payoff for subset in equation (5.30) exactly coincides with the lower bound. 
Corollary 5.3. For any $v \in I^{n}$ and $\mathscr{F}_{\chi}=\left\{x \in \mathbf{R}^{n} \mid x(N)=v(N), m_{i}^{\chi}(v) \leq x_{i} \leq\right.$ $\left.M_{i}^{\chi}(v), \forall i \in N\right\}$, the maximal potential payoff and minimal potential payoff for any $i \in N$ are given as

(i) $M_{i}^{\mathscr{F}_{\chi}}(v)=\min \left\{v(N)-\sum_{j \in N \backslash\{i\}} m_{j}^{\chi}(v), M_{i}^{\chi}(v)\right\}$.

(ii) $m_{i}^{\mathscr{F} \chi}(v)=m_{i}^{\chi}(v)$.

Proof. According to Theorem 5.6, we have

$$
\begin{aligned}
& M_{i}^{\mathscr{F} \chi}(v)=\min \left\{v(N)-\sum_{j \in N \backslash\{i\}} m_{j}^{\chi}(v), M_{i}^{\chi}(v)\right\}, \\
& m_{i}^{\mathscr{F} \chi}(v)=\max \left\{m_{i}^{\chi}(v), v(N)-\sum_{j \in N \backslash\{i\}} M_{j}^{\chi}(v)\right\} .
\end{aligned}
$$

Thus it remains to prove that $m_{i}^{\chi}(v) \geq v(N)-\sum_{j \in N \backslash\{i\}} M_{j}^{\chi}(v)$ for all $i \in N$, which has been verified by Bergantiños et al.(see Proposition 1, [8]).

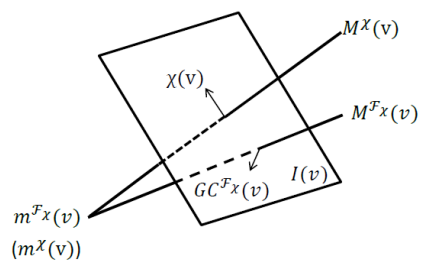

FIGURE 5.3: The general compromise value $G C^{\mathscr{F}} x(v)$ of game $v \in I^{n}$.

The $\chi$-value and the general compromise value are generated from the same lower bound, but the later possesses a lower upper bound. Recall that $m_{i}^{\chi}(v)=v(\{i\})$ for all $i \in N$ and $v \in I^{n}$, it follows that $v(N)-\sum_{j \in N \backslash\{i\}} m_{j}^{\chi}(v) \geq$ $v(\{i\})$. Together with the fact that $M_{i}^{\chi}(v) \geq v(\{i\})$, we conclude that $M_{i}^{\mathscr{F}}(v) \geq$ $v(\{i\})$ and $m_{i}^{\mathscr{F}_{\chi}}(v) \geq v(\{i\})$, thus we have $G C^{\mathscr{F}_{\chi}}(v) \in I(v)$. In this way, both the $\chi$-value and the general compromise value are the unique imputation in linear segments with different extreme points (see Figure 5.3). The next theorem reveals the conditions under which these two values are identical. 
Theorem 5.8. For any $v \in S I^{n}:=\left\{v \in \Gamma_{n} \mid v(S)-v(S \backslash\{i\}) \geq v(\{i\}), \forall S \subseteq\right.$ $N$ with $i \in S\}$ and $\mathscr{F}_{\chi}=\left\{x \in \mathbf{R}^{n} \mid x(N)=v(N), m_{i}^{\chi}(v) \leq x_{i} \leq M_{i}^{\chi}(v), \forall i \in\right.$ $N\}$, the general compromise value coincides with the $\chi$-value.

Proof. Let $v \in S I^{n}$, then

$$
v(N) \geq v(N \backslash\{i\})+v(\{i\}) \geq v(N \backslash\{i, j\})+v(\{j\})+v(\{i\}) \geq \cdots \geq \sum_{j \in N} v(\{j\}) .
$$

Hence we have $v \in I^{n}$, which means that $\mathscr{F}_{\chi}$ is not empty according to Proposition 5.4. In view of Corollary 5.3, it is sufficient to prove that $M^{\mathscr{F}} \chi(v)=$ $M^{\chi}(v)$, i.e.,

$$
v(N)-\sum_{j \in N \backslash\{i\}} m_{j}^{\chi}(v) \geq M_{i}^{\chi}(v) \text { for all } i \in N .
$$

In fact we can show that

$$
v(S)-\sum_{j \in S \backslash\{i\}} m_{j}^{\chi}(v) \geq M_{i}^{\chi}(v) \text { for all } S \subseteq N \text { with } i \in N \text { and }|S| \geq 2 .
$$

The proof is carried by induction on the cardinality of coalition $S$. When $S=\{i, j\}$, then

$$
v(S)-\sum_{j \in S \backslash\{i\}} m_{j}^{\chi}(v)=v(\{i, j\})-v(\{j\}) \geq v(\{i\}) .
$$

The last inequality is derived from $v \in S I^{n}$.

Supposing that Equation (5.35) holds for all $S$ with no more than $k$ players, then we show that the result is true for coalition with $k+1$ players. Given $S=\left\{i_{1}, i_{2}, \cdots, i_{k}, i\right\}$, then

$$
\begin{aligned}
v(S)-\sum_{j \in S \backslash\{i\}} m_{j}^{\chi}(v) & =v(S)-\sum_{j \in\{1,2, \cdots, k\}} m_{i_{j}}^{\chi}(v) \\
& \geq v(S)-\left[v(S)-v\left(S \backslash\left\{i_{k}\right\}\right)\right]-\sum_{j \in\{1,2, \cdots, k-1\}} m_{i_{j}}^{\chi}(v) \\
& =v\left(S \backslash\left\{i_{k}\right\}\right)-\sum_{j \in\{1,2, \cdots, k-1\}} m_{i_{j}}^{\chi}(v)
\end{aligned}
$$




$$
=v\left(S \backslash\left\{i_{k}\right\}\right)-\sum_{j \in S \backslash\left\{i_{k}, i\right\}} m_{i_{j}}^{\chi}(v)
$$

The inequality is due to the condition that $v \in S I^{n}$. Notice that $S \backslash\left\{i_{k}\right\}$ consists of $k$ players, thus by the induction hypothesis, we conclude that

$$
v(S)-\sum_{j \in S \backslash\{i\}} m_{j}^{\chi}(v) \geq M_{i}^{\chi}(v) \text { for all } S \subseteq N \text { with } i \in N \text { and }|S| \geq 2 .
$$

Particularly, let $S=N$, it follows that Equation (5.34) holds, which completes the proof.

\subsection{Characterization of the general compromise value}

Recall that Tijs [82] characterized the $\tau$-value with the aid of relative invariance under $S$-equivalence and the restricted proportionality. As a generalization of the $\tau$-value, the general compromise value can be determined by similar properties. As to the relative invariance under $S$-equivalence, the difference is that the positive affine transformations not only act on the games but also on the set of the set of potential payoffs. In view of Equation (5.8), it follows that the general compromise value is proportional to the maximal (minimal) potential payoff vector once the minimal (maximal) potential payoff vector turns into zero. The next theorem reveals that these two properties fully characterize the general compromise value together with the efficiency principle.

Theorem 5.9. For any $v \in \Gamma_{n}$ and nonempty bounded subset $\mathscr{F} \subseteq I^{*}(v)$, the general compromise value $G C^{\mathscr{F}}: v \rightarrow \mathbf{R}^{n}$ is the unique value $\phi$ on $\Gamma_{n}$ with the following three properties:

(i) efficiency principle,

(ii) relative invariance under $S$-equivalence: for all $\alpha \in(0,+\infty)$ and $d \in \mathscr{R}^{n}$, it follows that $\phi(\alpha v+d)=\alpha \phi(v)+d$ and

(iii)maximal proportional property: for any $v \in \Gamma_{n}$ satisfying $m^{\mathscr{F}}(v)=0$, the value vector $\phi(v)$ is proportional to the maximal potential payoff vector $M^{\mathscr{F}}(v)$. 
Proof. In view of Lemma 5.2, one could easily verify the validity of the relative invariance under $S$-equivalence. Efficiency principle and the third property holds based on the definition of the general compromise value. It remains to prove the uniqueness part.

Suppose that $\phi \rightarrow \mathbf{R}^{n}$ is a value satisfying the three mentioned properties, we show that $\phi=G C^{\mathscr{F}}$. Define the vector $d \in \mathbf{R}^{n}$ and the game $w \in \Gamma_{n}$ by $d:=m^{\mathscr{F}}(v)$ and $w:=v-d$. Since $\phi$ and $G C^{\mathscr{F}}$ both are relatively invariant under $S$-equivalence, we have $\phi(w)=\phi(v)-d$ and $G C^{\mathscr{F}^{\prime}}(w)=G C^{\mathscr{F}}(v)-d$, where $\mathscr{F}^{\prime}=\mathscr{F}-d$. To show that $\phi(v)=G C^{\mathscr{F}}(v)$, it is sufficient to show that $\phi(w)=G C^{\mathscr{F}^{\prime}}(w)$. Based on Lemma 5.2, for all $i \in N$, it holds that

$$
\begin{aligned}
& M_{i}^{\mathscr{F}^{\prime}}(w)=M_{i}^{\mathscr{F}^{\prime}}(v-d)=\sup _{x \in \mathscr{F}}\left\{x_{i}-d_{i}\right\}=M_{i}^{\mathscr{F}}(v)-m_{i}^{\mathscr{F}}(v), \\
& m_{i}^{\mathscr{F}^{\prime}}(w)=m_{i}^{\mathscr{F}^{\prime}}(v-d)=\inf _{x \in \mathscr{F}}\left\{x_{i}-d_{i}\right\}=m_{i}^{\mathscr{F}}(v)-m_{i}^{\mathscr{F}}(v)=0 .
\end{aligned}
$$

Notice that $w$ satisfies $m_{i}^{\mathscr{F}^{\prime}}(w)=0$, hence $\phi(w)$ and $G C^{\mathscr{F}^{\prime}}(w)$ are proportional to the maximal potential payoff vector $M^{\mathscr{F}^{\prime}}(w)$, which means that there exist real numbers $\beta$ and $\gamma$ such that

$$
\phi(w)=\beta M^{\mathscr{F}^{\prime}}(w), \quad G C^{\mathscr{F}^{\prime}}(w)=\gamma M^{\mathscr{F}^{\prime}}(w) .
$$

Obviously, $M_{i}^{\mathscr{F ^ { \prime }}}(w)=M_{i}^{\mathscr{F}}(v)-m_{i}^{\mathscr{F}}(v) \geq 0$ for all $i \in N$. If $\sum_{i \in N} M_{i}^{\mathscr{F}^{\prime}}(w)=0$, then $M_{i}^{\mathscr{F}^{\prime}}(w)=0$ for all $i \in N$ and therefore, we have $\phi(w)=G C^{\mathscr{F}^{\prime}}(w)$. When $\sum_{i \in N} M_{i}^{\mathscr{F}^{\prime}}(w)>0$, the efficiency principle of the two values implies that $\beta \sum_{i \in N} M_{i}^{\mathscr{F}^{\prime}}(w)=w(N)=\gamma \sum_{i \in N} M_{i}^{\mathscr{F}^{\prime}}(w)$ and hence, $\beta=\gamma$. Thus, we conclude that $\phi(w)=G C^{\mathscr{F}^{\prime}}(w)$ whenever $\sum_{i \in N} M_{i}^{\mathscr{F}^{\prime}}(w)>0$, which completes the proof.

Remark 5.4. According to the proof of Theorem 5.9, the relative invariance under $S$-equivalence can be replaced by a weaker property, that is,

- Minimal potential payoff property: for any $v \in \Gamma_{n}$ and nonempty bounded set $\mathscr{F} \subseteq I^{*}(v)$,

$$
G C^{\mathscr{F}}(v)=m^{\mathscr{F}}(v)+G C^{\mathscr{F}}\left(v-m^{\mathscr{F}}(v)\right) .
$$


Alternatively, the general compromise value can be characterized by means of the property that it is proportional to the minimal potential payoff vector once the maximal potential payoff vector equals zero, and we call this the minimal proportional property. The proof is a direct variation of Theorem 5.9 applied to $d:=M^{\mathscr{F}}(v)$, thus we omit the proof of the next theorem.

Theorem 5.10. For any $v \in \Gamma_{n}$ and nonempty subset $\mathscr{F} \subseteq I^{*}(v)$, the general compromise value $G C^{\mathscr{F}}: v \rightarrow \mathbf{R}^{n}$ is the unique value $\phi$ on $\Gamma_{n}$ with the following three properties:

(i) efficiency principle,

(ii) relative invariance under $S$-equivalence and

(iii) minimal proportional property: for any $v \in \Gamma_{n}$ satisfying $M^{\mathscr{F}}(v)=0$, the value vector $\phi(v)$ is proportional to the minimal potential payoff vector $m^{\mathscr{F}}(v)$. 



\section{Chapter 6}

\section{The optimal compromise values based on the}

lexicographic minimization criterion

In this chapter, we propose two kinds of complaints criteria based on which the optimistic (average) complaint and the pessimistic (average) complaint are defined. Two optimal compromise values are obtained by lexicographically minimizing the optimistic (average) complaint and the pessimistic (average) complaint, respectively. Interestingly, these two optimal compromise values coincide with the ENSC value and the CIS value, respectively. Moreover, these values are characterized in terms of equal maximal (average) complaint property and efficiency.

\subsection{Introduction}

The excess [19] proposed by Davis and Maschler in 1965 is one of the most important criteria to characterize the complaint with respect to a given payoff 
vector. The idea of excess forms the basis of several solution concepts for cooperative games in characteristic function form, such as the (pre-)kernel [19], the (pre-)nucleolus [71] and the $\tau$-value [83]. Beyond the excess criterion, there are other criteria to express the complaint. In this chapter, for every payoff vector $x$, two kinds of new criteria are proposed to measure the complaint of coalition $S, S \subseteq N$, with respect to the payoff vector $x$, by observing the fact that the marginal contribution vector and the individual worth vector are the upper bound and the lower bound of the core respectively. One is proposed from the perspective of coalitional insiders to measure the amount (the size of the inequity) by which the coalition falls short of its potential marginal contribution. While the other is based on the surplus of the complementary coalition, and the complaint is measured by the gap between the real payoff and the least potential payoff of the complementary coalition.

Instead of applying a general axiomatization of fairness to a value function defined on the set of all characteristic functions, we look at a fixed characteristic function $v$, and try to find a payoff vector $x$ that minimizes the maximal complaint under certain complaint criteria. Precisely, we look first at those coalitions $S$ whose complaint, for a fixed payoff vector $x$, is the largest. Then we adjust $x$, if possible, to make this largest complaint smaller. When the largest complaint has been made as small as possible, we concentrate on the next largest complaint, and adjust $x$ to make it as small as possible, and so on.

At first sight, the internal relationship between the ENSC value, the CIS value and the (pre-)nucleolus is not explicit. Driessen [24] revealed the coincidence of the ENSC value and the (pre-)nucleolus for restricted games in 1991. By minimizing the maximal complaint under different complaint criteria inspired by the definition of the (pre-)nucleolus, we obtain various significant solutions of the game, respectively. In the case coalition S's complaint is measured by the (average) gap of the sum of the grand marginal contributions and the proposed payoff vector $x$, the ENSC value is achieved by minimizing the optimistic maximal complaint. Alternatively, the coalition $S$ may take the (average) surplus of his complementary coalition $N \backslash S$ into consideration, which yields the other complaint criterion. To be precise, from a coalition point of view, since the players outside $S$ do not join $S$, it is assumed that the 
players $i \in N \backslash S$ do not cooperate and obtain their lowest potential payoff $v(i)$, which generates a gap between the proposed allocation $x_{i}$ and the individual value $v(i)$. When coalition $S$ 's complaint is measured by the (average) gap of its complementary coalition $N \backslash S$ 's least potential payoff and the given payoff vector, by minimizing the pessimistic maximal complaint, we achieve the CIS value.

\subsection{The optimal compromise value for cooperative games}

For any cooperative game $v \in \Gamma_{n}$ and payoff vector $x \in \mathbf{R}^{n}$, let $\theta(x)$ be the $2^{n}$ tuple whose components are the complaints of coalition $S, S \subseteq N$, arranged in non-increasing order, i.e.,

$$
\theta_{i}(x) \geq \theta_{j}(x), \quad \text { if } \quad 1 \leq i \leq j \leq 2^{n} .
$$

Here we leave $\theta(x)$ undefined but present $\theta(x)=\{v(S)-x(S)\}_{S \subseteq N}$ as an example. For any pair $x, y \in \mathbf{R}^{n}$, we have $x \leq_{L} y$, i.e. $x$ is lexicographically smaller than or equal to $y$, if $x=y$ or if there exists an $s \in\left\{1,2, \ldots, 2^{n}-1\right\}$ such that $x_{k}=y_{k}$ for all $k \in\{1,2, \ldots, s-1\}$ and $x_{s}<y_{s}$.

Definition 6.1. The optimal compromise value of a balanced game $v \in \Gamma_{n}$ is the pre-imputation $y \in I^{*}(v)$ of which the corresponding complaint vector $\theta(y)$ satisfies the lexicographic order $\theta(y) \leq_{L} \theta(x)$ for any $x \in I^{*}(v)$.

Obviously, under the complaint criterion of excess $v(S)-x(S)$, the optimal compromise value and the pre-nucleolus of the balanced game coincide. The question is: is there any other interesting or reasonable complaint criterion? Under different complaint criteria, what are the corresponding optimal compromise values? By answering these questions, we consider the bounds of the core.

To begin with, we focus on a balanced game $v$, of which the core is not empty and the elementary fact holds that for any $x \in C(v), v(i) \leq x_{i} \leq$ $v(N)-v(N \backslash i)$, that is, the grand marginal contribution vector and the individual worth vector are upper bound and lower bound of the core, respectively. 
The so-called optimal compromise value is based on the idea of minimizing the maximal complaint vector, while the optimistic optimal compromise value and the pessimistic optimal compromise value are related to the complaint vectors with respect to $\left\{b_{i}^{v}\right\}_{i \in N}$ and $\{v(i)\}_{i \in N}$, as defined below

Definition 6.2. For any balanced game $v$, the optimistic complaint and the pessimistic complaint of a coalition $S$ are given by

$$
e\left(S, x, b^{v}\right)=b^{v}(S)-x(S), S \subseteq N
$$

and

$$
e(S, x, v(\cdot))=x(N \backslash S)-\sum_{j \in N \backslash S} v(j), \emptyset \neq S \subseteq N .
$$

We define these two complaint criteria by "optimistic" and "pessimistic", since in the optimistic terms, players always take the surplus of themselves into consideration, while the pessimist always departs from the other players' extra payoff. The optimistic complaint $e\left(S, x, b^{v}\right)$ of coalition $S$ at the payoff vector $x$ with respect to the marginal contribution vector $b^{v}$ of the balanced game $v$, represents the amount of the complaint about the payoff distribution $x$, assuming the member $i$ in coalition $S$ is willing to obtain the monetary utility of which is measured by his marginal contribution $b_{i}^{v}=v(N)-v(N \backslash\{i\})$. For a coalition $S$, the lowest potential payoff of player $j$ in the complementary coalition $N \backslash S$ is his individual worth $v(j)$. Thus, the difference of the sum of the $N \backslash S$ 's payoff and the sum of the individual worths, i.e., $x(N \backslash S)-\sum_{j \in N \backslash S} v(j)$, is an alternative way to measure the complaint of coalition $S$. The pessimistic complaint $e(S, x, v(\cdot))$ represents the complaint of a non-empty coalition $S$ at the payoff vector $x$ with respect to the least potential payoff of his complementary coalition in the game $v$, assuming coalition $S$ only wants the outsiders get their individual worths.

In next subsections, we will determine the optimistic optimal compromise value and the pessimistic optimal compromise value based on the optimistic and pessimistic complaints. 


\subsubsection{The determination of the optimal optimistic compromise value}

Definition 6.3. The optimal optimistic compromise value of a balanced game $v$ is the pre-imputation $x$ of which the corresponding optimistic complaint vector $\theta(x)$ satisfies $\theta(x) \leq_{L} \theta(y)$ for any $y \in I^{*}(v)$.

Similar to the definition of the pre-nucleolus, the optimal optimistic compromise value minimizes the maximal optimistic complaint over all coalitions for a given pre-imputation. We reveal that such a value is bounded from above by the grand marginal contribution.

Proposition 6.1. Let $v$ be a balanced game, then the optimal optimistic compromise value $x$ is bounded from above by the grand marginal contribution vector $b^{v}$, i.e., $x_{i} \leq b_{i}^{v}, \forall i \in N$.

Proof. Suppose $x_{i} \leq b_{i}^{v}$ does not hold for all $i \in N$.

Case 1: If $x_{i} \geq b_{i}^{v}$ holds for all $i \in N$ and there exists at least one player $j, j \in N$ such that $x_{j}>b_{j}^{v}$, then

$$
\sum_{i \in N} x_{i}>\sum_{i \in N} b_{i}^{v} \geq v(N) .
$$

The last inequality holds because the marginal vector $b^{v}$ is the upper bound of the core. Thus, $x(N)>v(N)$, which contradicts the efficiency of the optimal optimistic compromise value.

Case 2: There exist at least two players, $i, j \in N$, such that $x_{i}>b_{i}^{v}, x_{j}<b_{j}^{v}$. Denote $\Delta=\min \left\{b_{j}^{v}-x_{j}, x_{i}-b_{i}^{v}\right\}$. Then $\triangle>0$ and for the new payoff $x^{*}$, where

$$
x_{k}^{*}= \begin{cases}x_{k} & \text { for any } k \neq i, j \\ x_{j}+\triangle & k=j \\ x_{i}-\triangle & k=i\end{cases}
$$

it holds $\theta(x)>_{L} \theta\left(x^{*}\right)$ by observing that the first coordinates of $\theta(x)$ and 
$\theta\left(x^{*}\right)$ are $\sum_{b_{j}^{v}>x_{j}, j \in N}\left(b_{j}^{v}-x_{j}\right)$ and $\sum_{b_{j}^{v}>x_{j}, j \in N}\left(b_{j}^{v}-x_{j}\right)-\triangle$, respectively, which contradicts the claim that $x$ is the optimal optimistic compromise value. Therefore, $x_{i} \leq b_{i}^{v}, \forall i \in N$.

Examining the proof of Proposition 6.1, we find that the conclusion also holds for a larger class of games $\Gamma_{1}=\left\{v \in \Gamma_{n} \mid v(N) \leqslant b^{v}(N)\right\}$, here we omit the proof.

Lemma 6.1. Given the optimistic complaint criterion, a payoff vector $x \in I^{*}(v)$ of $v \in \Gamma_{1}$ and any player $m, l \in N$ such that $b_{l}^{v}-x_{l}>b_{m}^{v}-x_{m} \geq 0$, denote $\triangle=\frac{b_{l}^{v}-x_{l}-\left(b_{m}^{v}-x_{m}\right)}{2}$. Then for the new payoff vector $x^{*}$ constructed in the form of Equation (6.3), the following statements hold.

(i) $e\left(S, x^{*}, b^{v}\right)=e\left(S, x, b^{v}\right), \forall S \subseteq N$ with $S \not \supset m, l$ or $S \ni m, l$.

(ii) $e\left(S, x^{*}, b^{v}\right)<e\left(S, x, b^{v}\right), \forall S \subseteq N$ with $S \not \ngtr m, S \ni l$.

(iii) $\theta(x)>\theta\left(x^{*}\right)$.

Proof. It is not difficult to verify (i)-(ii), here we only prove (iii). For any $S \subseteq N$ satisfying $S \ni m, S \not \supset l$, we have

$$
\begin{aligned}
& e\left(S, x, b^{v}\right)=b^{v}(S)-x(S) \\
= & b^{v}(S \backslash\{m\})-x(S \backslash\{m\})+\left(b_{m}^{v}-x_{m}\right) \\
< & b^{v}(S \backslash\{m\})-x(S \backslash\{m\})+\left(b_{l}^{v}-x_{l}\right) \\
= & b^{v}(S \cup\{l\} \backslash\{m\})-x(S \cup\{l\} \backslash\{m\})=e\left(S \cup\{l\} \backslash\{m\}, x, b^{v}\right) .
\end{aligned}
$$

Which means that for any $S \ni m, S \not \supset l$, there always exists coalition $S \cup$ $\{l\} \backslash\{m\}$, such that $e\left(S \cup\{l\} \backslash\{m\}, x, b^{v}\right)>e\left(S, x, b^{v}\right)$. On the other, note that $b_{l}^{v}-x_{l}^{*}=b_{m}^{v}-x_{m}^{*}$, thus,

$$
\begin{aligned}
& e\left(S, x^{*}, b^{v}\right)=b^{v}(S)-x^{*}(S) \\
= & b^{v}(S \backslash\{m\})-x^{*}(S \backslash\{m\})+\left(b_{m}^{v}-x_{m}^{*}\right) \\
= & b^{v}(S \backslash\{m\})-x(S \backslash\{m\})+\left(b_{l}^{v}-x_{l}^{*}\right) \\
= & b^{v}(S \cup\{l\} \backslash\{m\})-x^{*}(S \cup\{l\} \backslash\{m\})=e\left(S \cup\{l\} \backslash\{m\}, x^{*}, b^{v}\right) .
\end{aligned}
$$


Therefore, in order to lexicographically compare $\theta(x)$ and $\theta\left(x^{*}\right)$, it is sufficient to compare $e\left(S \cup\{l\} \backslash\{m\}, x^{*}, b^{v}\right)$ and $e\left(S \cup\{l\} \backslash\{m\}, x, b^{v}\right)$. According to (ii), we have $e\left(S \cup\{l\} \backslash\{m\}, x^{*}, b^{v}\right)<e\left(S \cup\{l\} \backslash\{m\}, x, b^{v}\right)$, which implies that $\theta(x)>\theta\left(x^{*}\right)$.

Theorem 6.2. If $x$ is the optimal optimistic complaint value of game $v \in \Gamma_{1}$, then $b_{i}^{v}-x_{i}=b_{m}^{v}-x_{m}, \forall i, m \in N$ and $x_{i}=\operatorname{ENSC}_{i}(v), \forall i \in N$.

Proof. (i) Suppose there exist $l, m$ such that $b_{l}^{v}-x_{l} \neq b_{m}^{v}-x_{m}$. Without loss of generality, let $b_{l}^{v}-x_{l}>b_{m}^{v}-x_{m}$. By Lemma 6.1, there exists $x^{*}$ such that $\theta(x)>\theta\left(x^{*}\right)$, which is in contradiction with that $x$ is the optimal optimistic compromise value. Therefore, $b_{i}^{v}-x_{i}, \forall i \in N$, is constant.

(ii) It is easy to obtain that $x_{i}=E N S C_{i}(v)=b_{i}^{v}+\frac{1}{n}\left[v(N)-\sum_{j \in N} b_{j}^{v}\right]$ by (i) together with efficiency.

Remark 6.1. In fact there is also an alternative way to determine the optimal optimistic compromise value. Recall that a cooperative game $v \in \Gamma_{n}$ is called 1-convex [21,26] if it satisfies $b^{v}(N) \geq v(N)$ and $v(S) \leq v(N)-$ $b^{v}(N \backslash S), \forall S \subseteq N, S \neq \emptyset$. The 1-convex condition states that the amount which remains in the game $v$ for a coalition $S$, when the total amount $v(N)$ is distributed in such a way that all the players outside $S$ obtain their marginal contributions, is at least its worth $v(S)$. For the 1-convex game, the core is not empty, i.e., the 1-convex game is balanced. The nucleolus of a 1-convex $n$-person game has already been determined by Driessen [22] as $N u_{i}^{v}=b_{i}^{v}-$ $\frac{b^{v}(N)-v(N)}{n}=E N S C_{i}(v)$. For any $v \in \Gamma_{1}$, the optimal optimistic compromise value can be viewed as the nucleolus of the corresponding additive game $\bar{v}(S)=b^{v}(S), \forall S \subseteq N$. Notice that any additive game is also 1-convex, which immediately give rise to the validity of Theorem 6.2.

\subsubsection{The determination of the optimal pessimistic compromise value}

In Subsection 6.2.1, we implement the ENSC value by minimizing the maximal complaint under the optimistic complaint criterion. In this section, we will deal with the CIS value which is anti-dual to the ENSC value. 
Definition 6.4. The optimal pessimistic compromise value of a balanced game $v$ is the pre-imputation $x$ of which the corresponding pessimistic complaint vector $\theta(x)$ satisfies $\theta(x) \leq_{L} \theta(y) \forall y \in I^{*}(v)$.

Proposition 6.2. Let $v$ be a balanced game. The optimal pessimistic compromise value $x$ is bounded from below by individual vector, i.e., $x_{i} \geq v(i), \forall i \in$ $N$. That is, the pessimistic optimal compromise value belongs to the imputation set.

Proof. Suppose $x_{i} \geq v(i)$ does not hold for all $i \in N$.

Case 1: If $x_{i} \leq v(i)$ holds for all $i \in N$, then there exists at least one player $j$ such that $x_{j}<v(j)$, then

$$
x(N)<\sum_{i \in N} v(i) \leq v(N) .
$$

The last inequality holds since the individual worth is a lower bound of the core. Thus, $x(N)<v(N)$, which is in contradiction with the efficiency of the optimal pessimistic compromise value.

Case 2: There exist at least two players, $i, j \in N$, such that $x_{i}\left\langle v(i), x_{j}\right\rangle$ $v(j)$. Denote $\Delta=\min \left\{x_{j}-v(j), v(i)-x_{i}\right\}$, then $\Delta>0$ and for the new payoff $x^{*}$ constructed in the form of (6.3), it holds $\theta(x)>\theta\left(x^{*}\right)$, because the first coordinates of $\theta(x)$ and $\theta\left(x^{*}\right)$ are $\theta_{1}(x)=\sum_{x_{j}>v(j), j \in N}\left(b_{j}-x_{j}\right)$ and $\theta_{1}\left(x^{*}\right)=$ $\sum_{x_{j}>v(j), j \in N}\left(b_{j}-x_{j}\right)-\triangle$, respectively, yielding $\theta_{1}(x)>\theta_{1}\left(x^{*}\right)$. This contradicts the claim that $x$ is the optimal pessimistic compromise value. Therefore, $x_{i} \geq$ $v(i), \forall i \in N$.

Examining the proof of Proposition 6.2, we find that the conclusion also holds for a larger class of games $\Gamma_{2}=\left\{v \in \Gamma_{n} \mid v(N) \geq \sum_{k \in N} v(k)\right\}$, here we omit the proof.

Lemma 6.3. Given the pessimistic complaint criterion, the imputation payoff vector $x$ of the game $v \in \Gamma_{2}$ and any player $m$ such that $x_{l}-v(l)>x_{m}-v(m)$, $l, m \in N$, denote $\Delta=\frac{x_{l}-v(l)-\left(x_{m}-v(m)\right)}{2}$. Then for the new payoff vector $x^{*}$ constructed in the form of Equation (6.3), it holds that $\theta(x)>\theta\left(x^{*}\right)$. 
Theorem 6.4. If $x$ is the optimal pessimistic compromise value of a game $v \in \Gamma_{2}$, then $x_{i}-v(i)=x_{m}-v(m), \forall i, m \in N$ and $x_{i}=C I S_{i}(v), \forall i \in N$.

The proof of Lemma 6.3 and Theorem 6.4 is similar to that of Lemma 6.1 and Theorem 6.2, respectively, based on the fact that the CIS value and the ENSC value are dual [58]. Actually, the validity of Theorem 6.4 can also be shown by introducing the anti-dual of cooperative games, which is a useful tool to analyze the relationship between the properties and the solutions [58].

Definition 6.5. For any cooperative game $v$, the anti-dual of $v \in \Gamma_{n}$, denoted by $v^{\star}$, is defined by

$$
v^{\star}(S)=-[v(N)-v(N \backslash S)] \text {, for all } S \subseteq N .
$$

The value $-v^{\star}$ indicates the amount that the complement coalition $N \backslash S$ can not prevent $S$ from obtaining in $v$. By applying this "anti" operator, we reveal the relationship between the optimistic optimal compromise value and the pessimistic optimal compromise value.

Theorem 6.5. If $x$ is the optimal pessimistic compromise value of the game $v \in \Gamma_{2}$, then

(i) $x^{\star}$ is the optimal optimistic compromise value of the anti-dual game $v^{\star}$, where $x^{\star}=-x$.

(ii) $\forall i \in N, x_{i}-v(i)$ is constant and $x_{i}=C I S_{i}(v)$.

Proof. (i) Since $x$ is the optimal pessimistic compromise value of the game $v \in \Gamma_{2}, \theta(x, v) \leq_{L} \theta(y, v)$, for any $y \in I^{*}(v)$. Note that $v^{\star}(S)=-[v(N)-$ $v(N \backslash S)$ ], then

$e\left(N \backslash S, x^{\star}, b^{v^{\star}}\right)=b^{v^{\star}}(N \backslash S)-x^{\star}(N \backslash S)=x(N \backslash S)-\sum_{i \in N \backslash S} v(i)=e(S, x, v(\cdot))$.

Therefore, $\theta\left(x^{\star}, v^{\star}\right) \leq_{L} \theta\left(y^{\star}, v^{\star}\right)$ for all $y^{\star}=-y \in I^{*}\left(v^{\star}\right)$, i.e., $x^{\star}$ is the optimal optimistic compromise value for game $v^{\star}$.

(ii) As shown in (i), $x^{\star}$ is the optimal optimistic compromise value for game $v^{\star}$. By theorem 6.2(i), $b_{i}^{v^{\star}}-x_{i}^{\star}$ is constant and $x_{i}^{\star}=b_{i}^{v^{\star}}+\frac{1}{n}\left[v^{\star}(N)-\sum_{k \in N} b_{k}^{v^{\star}}\right]$. On the other hand, we have $v(i)=-b_{i}^{v^{\star}}$ and $x^{\star}=-x$. Hence, $b_{i}^{v^{\star}}-x_{i}^{\star}=x_{i}-$ 
$v(i), \forall i \in N$ is constant. And $x_{i}=-x_{i}^{\star}=v(i)+\frac{1}{n}\left[v(N)-\sum_{j \in N} v(j)\right]$, which holds by replacing $b_{i}^{v^{\star}}$ and $v^{\star}(N)$ with $-v(i)$ and $-v(N)$ respectively.

\subsubsection{The determination of the optimal average compromise value}

The nucleolus is defined by means of the maximal dissatisfaction of coalitions. Notice that the nucleolus ignores the size of coalitions when comparing the complaint. Coalitions with more members do usually have more complaint compared with coalitions with smaller size, but this does not mean that the individual players have more complaints. Based on such consideration, Grotte [32] introduced the per capita nucleolus by means of the maximal dissatisfaction per player for every coalition. In this section, similarly, we will take into account the cardinality of coalitions and investigate the solution concepts generated by the so-called average optimistic and pessimistic complaint.

Definition 6.6. For any balanced game $v$, the optimistic average complaint and the pessimistic average complaint of coalition $S$ are given by

$$
\begin{gathered}
\bar{e}\left(S, x, b^{v}\right)=\frac{b^{v}(S)-x(S)}{|S|}, \emptyset \neq S \subseteq N, \\
\bar{e}(S, x, v\{(\cdot)\})=\frac{x(N \backslash S)-\sum_{j \in N \backslash S} v(j)}{|N \backslash S|}, S \varsubsetneqq N .
\end{gathered}
$$

The optimistic average complaint $\bar{e}\left(S, x, b^{v}\right)$ of coalition $S$ demonstrates the amount of the per capita complaint of $S$ at the payoff distribution $x$, assuming all the players in coalition $S$ are willing to obtain their marginal contributions. It is an alternative way to measure the complaint of coalition $S$ by the average gap between the complementary coalition $N \backslash S$ 's payoff and the sum of the individual worth of the players, by observing that the players outside coalition $S$ do not cooperate with each other. The pessimistic average complaint $\bar{e}(S, x, v(\cdot))$ represents the per capita complaint at the payoff vector $x$ from the complementary coalitional point of view. Similarly, we consider 
the solution that lexicographically minimizes the corresponding complaint vector.

Definition 6.7. The optimal optimistic average compromise value of a balanced game $v$ is the pre-imputation $x$ of which the corresponding optimistic average complaint vector $\theta(x)$ satisfies $\theta(x) \leq_{L} \theta(y), \forall y \in I^{*}(v)$.

Definition 6.8. The optimal pessimistic average compromise value of a balanced game $v$ is the pre-imputation $x$ of which the corresponding pessimistic average complaint vector $\theta(x)$ satisfies $\theta(x) \leq_{L} \theta(y), \forall y \in I^{*}(v)$.

It turns out that both these two optimal average compromise values have the same bound as we have discussed earlier.

Theorem 6.6. For any balanced game $v$, the optimal optimistic average compromise value $x$ is bounded from above by $b^{v}$, i.e., $x_{i} \leq b_{i}^{v}, \forall i \in N$.

Proof. Suppose $x_{i} \leq b_{i}^{v}$ is not true for all $i \in N$.

Case 1: If $x_{i} \geq b_{i}^{v}$ holds for all $i \in N$, there exists at least one player $j$ such that $x_{j}>b_{j}$, then

$$
\sum_{i \in N} x_{i}>\sum_{i \in N} b_{i}^{v} \geq v(N)
$$

where the last inequality holds because $b^{v}$ is the upper bound of the Core. Therefore, $x(N)>v(N)$, which yields a contradiction with the efficiency of the upper optimal complaint value.

Case 2: There exist at least two players $s, t \in N$, such that $x_{s}<b_{s}^{v}, x_{t}>b_{t}^{v}$, denote $T_{1}=\left\{k \in N \mid b_{k}^{v}-x_{k} \geq b_{l}^{v}-x_{l}, \forall l \in N\right\}$ and $T_{2}=\left\{k \in N \backslash T_{1} \mid b_{k}^{v}-x_{k} \geq\right.$ $\left.b_{l}^{v}-x_{l}, \forall l \in N \backslash T_{1}\right\}$. Then $T_{1} \neq T_{2} \neq \emptyset$ and for any $p \in T_{1}, q \in T_{2}, p, q$ are the players whose complaint are the first and second largest respectively under the optimistic average complaint criterion. Denote $\triangle=\min \left\{b_{p}^{v}-x_{p}, x_{t}-b_{t}^{v}\right\}$, then $\Delta>0$ and construct the new payoff $x^{*}$, where

$$
x_{k}^{*}=\left\{\begin{array}{l}
x_{k} \quad \text { for all } k \neq t \text { and } k \notin T_{1} ; \\
x_{k}+\frac{\Delta}{\left|T_{1}\right|}, \quad k \in T_{1} ; \\
x_{k}-\triangle, \quad k=t .
\end{array}\right.
$$


Then we have

$$
\begin{aligned}
b_{t}^{v}-x_{t}^{*} & =b_{t}^{v}-x_{t}+\Delta=b_{t}^{v}-x_{t}+\min \left\{b_{p}^{v}-x_{p}, x_{t}-b_{t}^{v}\right\} \\
& =\min \left\{b_{t}^{v}-x_{t}+b_{p}^{v}-x_{p}, 0\right\} \leq 0
\end{aligned}
$$

Subcase 1 If $t \notin T_{2}$, then for any $p \in T_{1}$

$$
\begin{gathered}
b_{p}^{v}-x_{p}^{*}=b_{p}^{v}-x_{p}-\frac{\triangle}{\left|T_{1}\right|}=b_{p}^{v}-x_{p}-\frac{\min \left\{b_{p}^{v}-x_{p}, x_{t}-b_{t}^{v}\right\}}{\left|T_{1}\right|} \geq 0, \text { yielding } \\
b_{p}^{v}-x_{p}^{*} \geq b_{t}^{v}-x_{t}^{*} .
\end{gathered}
$$

On the other hand, for all $s \in N \backslash\left(T_{1} \cup T_{2} \cup\{t\}\right), q \in T_{2}$, by (6.7), it is easy to obtain

$$
b_{q}^{v}-x_{q}^{*}=b_{q}^{v}-x_{q}>b_{s}^{v}-x_{s}=b_{s}^{v}-x_{s}^{*}, \forall q \in T_{2},
$$

where the inequality holds because $q \in T_{2}$ is the player whose complaint is the second largest, while $s$ is the player whose complaint is less that that of $q \in T_{2}$ and $p \in T_{1}$. Note that $N=N \backslash\left(T_{1} \cup T_{2} \cup\{t\}\right) \cup T_{1} \cup T_{2} \cup\{t\}$. Therefore, by (6.9) and (6.10),

$$
\begin{aligned}
\theta_{1}\left(x^{*}\right) & =\max _{k \in N}\left\{b_{k}^{v}-x_{k}^{*}\right\} \\
& =\max _{p \in T_{1}, q \in T_{2}}\left\{b_{p}^{v}-x_{p}^{*}, b_{q}^{v}-x_{q}^{*}\right\} \\
& =\max _{p \in T_{1}, q \in T_{2}}\left\{b_{p}^{v}-x_{p}-\frac{\triangle}{\left|T_{1}\right|}, b_{q}^{v}-x_{q}\right\} \\
& \leq b_{p}^{v}-x_{p} \\
& =\theta_{1}(x) .
\end{aligned}
$$

Therefore, $\theta(x)>\theta\left(x^{*}\right)$, which yields a contradiction with the statement that $x$ is the optimal optimistic average complaint value.

Subcase 2. if $t \in T_{2}$, then by (6.8) and (6.9), it is easy to obtain that

$$
b_{p}^{v}-x_{p}^{*} \geq 0 \geq b_{q}^{v}-x_{q}^{*}, \forall p \in T_{1} \text { and } \forall q \in T_{2} .
$$


Notice that $N=N \backslash\left(T_{1} \cup T_{2}\right) \cup T_{1} \cup T_{2}$. Hence, by (6.11),

$$
\begin{aligned}
\theta_{1}\left(x^{*}\right) & =\max _{k \in N}\left\{b_{k}^{v}-x_{k}^{*}\right\} \\
& =\max _{p \in T_{1}, q \in T_{2}, s \in N \backslash\left(T_{1} \cup T_{2}\right)}\left\{b_{p}^{v}-x_{p}^{*}, b_{q}^{v}-x_{q}^{*}, b_{s}^{v}-x_{s}^{*}\right\} \\
& =\max _{p \in T_{1}, s \in N \backslash\left(T_{1} \cup T_{2}\right)}\left\{b_{p}^{v}-x_{p}^{*}, b_{s}^{v}-x_{s}^{*}\right\} \\
& =\max _{p \in T_{1}, s \in N \backslash\left(T_{1} \cup T_{2}\right)}\left\{b_{p}^{v}-x_{p}-\frac{\triangle}{\left|T_{1}\right|}, b_{s}^{v}-x_{s}\right\} \\
& <b_{p}^{v}-x_{p} \\
& =\theta_{1}(x),
\end{aligned}
$$

which implies $\theta\left(x^{*}\right)<\theta(x)$. This contradicts that $x$ is the optimal optimistic average complaint value. Thus, $x_{i} \leq b_{i}^{v}, \forall i \in N$.

Theorem 6.7. For any balanced game $v$, the optimal pessimistic average compromise value $x$ is bounded from below by individual worth, i.e., $x_{i} \geq v(i), \forall i \in$ $N$.

Proof. Suppose $x_{i} \geq v(i)$ does not hold for all $i \in N$.

Case 1. If $x_{i} \leq v(i)$ for all $i \in N$, there exists at least one player $k$ such that $x_{k}<v(k)$, then

$$
x(N)<\sum_{i \in N} v(i) \leq v(N)
$$

where the last inequality holds because the Core is lower bounded by the individual worth vector. Therefore, $x(N)<v(N)$, contradicting the efficiency of the optimal pessimistic average complaint value.

Case 2. There exist at least two players, $s, t \in N$, such that $x_{s}\left\langle v(s), x_{t}\right\rangle$ $v(t)$. Let $Q_{1}=\left\{k \in N \mid x_{k}-v(k) \geq x_{l}-v(l), \forall l \in N\right\}$ and $Q_{2}=\left\{k \in N \backslash Q_{1} \mid x_{k}-\right.$ $\left.v(k) \geq x_{l}-v(l), \forall l \in N \backslash Q_{1}\right\}$, then $Q_{1} \neq Q_{2} \neq \emptyset$. For any $p \in Q_{1}$, denote $\triangle=\min \left\{x_{p}-v(p), v(s)-x_{s}\right\}$, then $\triangle>0$. Construct the new payoff $x^{*}$ by

$$
x_{k}^{*}=\left\{\begin{array}{l}
x_{k}, \quad \text { for all } k \neq s \text { and } k \notin Q_{1} ; \\
x_{k}-\frac{\Delta}{\left|Q_{1}\right|}, \quad k \in Q_{1} \\
x_{s}+\triangle, \quad k=s
\end{array}\right.
$$


We then have

$$
\begin{aligned}
x_{s}^{*}-v(s) & =x_{s}+\Delta-v(s)=x_{s}-v(s)+\min \left\{x_{p}-v(p), v(s)-x_{s}\right\} \\
& =\min \left\{x_{s}-v(s)+\left(x_{p}-v(p)\right), 0\right\} \leq 0 .
\end{aligned}
$$

Subcase 1. If $s \notin Q_{2}$, for any $p \in Q_{1}$

$$
\begin{aligned}
x_{p}^{*}-v(p) & =x_{p}-v(p)-\frac{\Delta}{\left|Q_{1}\right|} \\
& =x_{p}-v(p)-\frac{\min \left\{x_{p}-v(p), v(s)-x_{s}\right\}}{\left|Q_{1}\right|} \geq 0 .
\end{aligned}
$$

Thus, $x_{p}^{*}-v(p) \geq x_{s}^{*}-v(s)$, yielding that

$$
\begin{aligned}
\theta_{1}\left(x^{*}\right) & =\max _{k \in N}\left\{x_{k}-v(k)\right\} \\
& =\max _{p \in Q_{1}, q \in Q_{2}}\left\{x_{p}^{*}-v(p), x_{q}^{*}-v(q)\right\} \\
& =\max _{p \in Q_{1}, q \in Q_{2}}\left\{x_{p}-v(p)-\frac{\triangle}{\left|Q_{1}\right|}, x_{q}-v(q)\right\} \\
& \leqslant x_{p}-v(p) .
\end{aligned}
$$

Therefore, $\theta(x)>\theta\left(x^{*}\right)$, which contradicts that $x$ is the optimal pessimistic average complaint value.

Subcase 2. If $s \notin Q_{2}$, then by (6.14) and (6.15), it holds that

$$
x_{p}^{*}-v(p) \geq 0 \geq x_{q}^{*}-v(q), \forall p \in Q_{1} \text { and } \forall q \in Q_{2},
$$

yielding that

$$
\begin{aligned}
\theta_{1}\left(x^{*}\right) & =\max _{k \in N}\left\{x_{k}-v(k)\right\} \\
& =\max _{p \in Q_{1}, j \in N \backslash\left(Q_{1} \cup Q_{2}\right)}\left\{x_{p}-v(p)-\frac{\triangle}{\left|Q_{1}\right|}, x_{j}-v(j)\right\} \\
& <x_{p}-v(p) .
\end{aligned}
$$

Thus, $\theta(x)>\theta\left(x^{*}\right)$, contradicting that $x$ is the optimal pessimistic average complaint value. Therefore, $x_{i} \geq v(i), \forall i \in N$. 
In fact, these two bounds can be extended to a more general class of games, $\Gamma_{1}$ and $\Gamma_{2}$ respectively. The proofs are similar to that of Theorems 6.6 and 6.7.

Theorem 6.8. For any game $(N, v) \in \Gamma_{1}$, the optimal optimistic average compromise value $x$ is bounded from above by $b^{v}$, i.e., $x_{i} \leq b_{i}^{v}, i \in N$.

Theorem 6.9. For any game $(N, v) \in \Gamma_{2}$, the optimal pessimistic average compromise value $x$ is bounded from below by individual worth, i.e., $x_{i} \geq v(i), i \in$ $N$.

By Theorem 6.8, we can narrow the scope of the optimistic optimal average compromise value from the pre-imputation set $I^{*}(v)$ to the set $K(v)=$ $\left\{x \mid x(N)=v(N), x_{i} \leq b_{i}^{v}\right\}$. The next lemma indicates the difference of the optimistic average complaint for each coalition when the payoff vector $x \in K(v)$ changes.

Lemma 6.10. For any game $v \in \Gamma_{1}$, given the optimistic average complaint criterion, the payoff vector $x \in K$ and any players $m, l \in N$ such that $b_{l}^{v}-x_{l}>$ $b_{m}^{v}-x_{m} \geq 0$, denote $\Delta=\frac{b_{l}^{v}-x_{l}-\left(b_{m}^{v}-x_{m}\right)}{2}$. Then for the new payoff vector $x^{*}$ where

$$
x_{k}^{*}=\left\{\begin{array}{l}
x_{k}, \quad \text { for all } k \neq l, m \\
x_{l}+\Delta, \quad k=l, \\
x_{m}-\triangle, \quad k=m .
\end{array}\right.
$$

the following five statements hold.

(i) $\bar{e}\left(S, x^{*}, b^{v}\right)=\bar{e}\left(S, x, b^{v}\right), \forall S \subseteq N$ with $S \not \nexists m, l$ or $S \ni m, l$.

(ii) $\bar{e}\left(S, x^{*}, b^{v}\right)<\bar{e}\left(S, x, b^{v}\right), \forall S \subseteq N$ with $S \not \supset m, S \ni l$.

(iii) $\theta(x)>\theta\left(x^{*}\right)$.

Proof. (i) and (ii) are easy to verify, here we provide the proof of (iii).

For any $S \subseteq N$ satisfying $S \ni m, S \not \ngtr l$, we have

$$
\begin{aligned}
\bar{e}\left(S, x, b^{v}\right) & =\frac{b^{v}(S)-x(S)}{|S|} \\
& =\frac{b^{v}(S \backslash\{m\})-x(S \backslash\{m\})+\left(b_{m}^{v}-x_{m}\right)}{|S|}
\end{aligned}
$$




$$
\begin{aligned}
& <\frac{b^{v}(S \backslash\{m\})-x(S \backslash\{m\})+\left(b_{l}^{v}-x_{l}\right)}{|S|} \\
& =\frac{b^{v}(S \cup\{l\} \backslash\{m\})-x(S \cup\{l\} \backslash\{m\})}{|S|} \\
& =\bar{e}\left(S \cup\{l\} \backslash\{m\}, x, b^{v}\right) .
\end{aligned}
$$

Therefore, for any $S \ni m, S \not \supset l$, there always exists coalition $S \cup\{l\} \backslash\{m\}$, such that $\bar{e}\left(S \cup\{l\} \backslash\{m\}, x, b^{v}\right)>\bar{e}\left(S, x, b^{v}\right)$. On the other, note that $b_{l}^{v}-x_{l}^{*}=$ $b_{m}^{v}-x_{m}^{*}$, thus,

$$
\begin{aligned}
\bar{e}\left(S, x^{*}, b^{v}\right) & =\frac{b^{v}(S)-x^{*}(S)}{|S|} \\
& =\frac{b^{v}(S \backslash\{m\})-x^{*}(S \backslash\{m\})+\left(b_{m}^{v}-x_{m}^{*}\right)}{|S|} \\
& =\frac{b^{v}(S \backslash\{m\})-x^{*}(S \backslash\{m\})+\left(b_{l}^{v}-x_{l}^{*}\right)}{|S|} \\
& =\frac{b^{v}(S \cup\{l\} \backslash\{m\})-x^{*}(S \cup\{l\} \backslash\{m\})}{|S|} \\
& =\bar{e}\left(S \cup\{l\} \backslash\{m\}, x^{*}, b^{v}\right) .
\end{aligned}
$$

By (i), (ii), Equations (6.18) and (6.19), it is sufficient to consider the relation of $\bar{e}\left(S, x, b^{v}\right)$ and $\bar{e}\left(S, x^{*}, b^{v}\right)$ when comparing $\theta(x)$ and $\theta\left(x^{*}\right)$ in lexicographical order. According to (ii), we have $\bar{e}\left(S \cup\{l\} \backslash\{m\}, x^{*}, b^{v}\right)<\bar{e}(S \cup$ $\left.\{l\} \backslash\{m\}, x, b^{v}\right)$, which implies that $\theta(x)>\theta\left(x^{*}\right)$.

As to the optimal pessimistic average complaint value, we can derive the same result.

Lemma 6.11. For any game $v \in \Gamma_{2}$, given the pessimistic average complaint criterion, the imputation payoff vector $x$ and any player $m$ such that $x_{m}-v(\{m\})>$ $x_{l}-v(\{l\}), l, m \in N$, denote $\Delta=\frac{x_{m}-v(\{m\})-\left(x_{l}-v(\{l\})\right)}{2}$, then for the new payoff vector $x^{*}$ constructed in the form of Equation (6.17), it holds that $\theta(x)>\theta\left(x^{*}\right)$.

With Lemma 6.10 and 6.11, we can prove that these two average compromise values coincide with the ENSC value ane the CIS value respectively. Since the proofs are similar to that of Theorems 6.2 and 6.5, here we omit them. 
Theorem 6.12. For any game $v \in \Gamma_{1}$, the optimal optimistic average compromise value coincides with the ENSC value.

Theorem 6.13. For any game $v \in \Gamma_{2}$, the optimal pessimistic average compromise value coincides with the CIS value.

\subsection{Characterization of the optimal compromise value}

The pre-kernel was introduced by Maschler, Peleg and Shapley in 1972 based on the ideas of excess and maximum surplus [48]. Analogously to this notion, we introduce the equal optimistic maximal complaint property and equal pessimistic maximal complaint property, by which we characterize the ENSC value and the CIS value respectively.

\subsubsection{Characterization of the optimal optimistic compromise value}

In order to characterize the optimal optimistic compromise value, we define the optimistic maximal complaint of player $i$ over player $j$.

Definition 6.9. For any $v \in \Gamma_{1}$ and $x \in I^{*}(v)$, the optimistic maximal complaint $m_{i j}^{v}(x)$ of player $i \in N$ over another player $j \in N$ at payoff vector $x$ is given by the maximal optimistic complaint among coalitions containing player $i$, but not containing player $j$ i.e.,

$$
m_{i j}^{v}\left(b^{v}, x\right)=\max \left[e\left(S, b^{v}, x\right) \mid S \subseteq N, i \in S, j \notin S\right] .
$$

A non-negative (non-positive) optimistic maximal complaint of player $i$ over $j$ at a given payoff vector reflects the maximal (minimal) amount that $i$ can obtain (lose) without cooperating with $j$ by withdrawing from the proposed payoff and establishing a coalition without $j$, in the understanding that the others of the new coalition agree with the allocation given by the initial payoff. Therefore the optimistic maximal complaint of player $i$ over $j$ can be viewed as the measure of the bargaining power of $i$ to negotiate with player $j$. For a reasonable solution concept, it is natural to require that no player 
could outweigh the others with respect to the final payoff vector. Hence we consider the following property: a value $\varphi: \Gamma_{1} \rightarrow \mathbf{R}^{n}$ satisfies

- equal optimistic maximal complaint property: if

$$
m_{i j}^{v}\left(b^{v}, \varphi(v)\right)=m_{j i}^{v}\left(b^{v}, \varphi(v)\right) \text {, for any } v \in \Gamma_{1}, i, j \in N,
$$

Lemma 6.14. For any $v \in \Gamma_{1}$, if $x$ is a payoff vector satisfying efficiency and the equal optimistic maximal complaint property, it holds that $b_{i}^{v} \geq x_{i}, \forall i \in N$.

Proof. We prove the lemma by showing that the following two claims do not hold.

Claim 1: $b_{i}^{v}<x_{i}$, for all $i \in N$.

Since $b_{i}^{v}<x_{i}, i \in N$, thus,

$$
\sum_{i \in N} b_{i}^{v}<\sum_{i \in N} x_{i}=v(N)
$$

which contradicts with $v \in \Gamma_{1}$. Therefore, claim 1 does not hold.

Claim 2: There exist at least two players $i, j \in N$, such that $b_{i}^{v}<x_{i}$ and $b_{j}^{v}>x_{j}, i, j \in N$.

By formula (6.20),

$$
\begin{aligned}
m_{i j}^{v}\left(b^{v}, x\right) & =\max \left[e\left(S, b^{v}, x\right) \mid \emptyset \neq S \subseteq N, i \in S, j \notin S\right] \\
& =\sum_{b_{k}^{v} \geq x_{k}, k \in N \backslash\{i, j\}}\left(b_{k}^{v}-x_{k}\right)+b_{i}^{v}-x_{i}, \\
m_{j i}^{v}\left(b^{v}, x\right) & =\max \left[e\left(S, b^{v}, x\right) \mid S \subseteq N, j \in S, i \notin S\right] \\
& =\sum_{b_{k}^{v} \geq x_{k}, k \in N \backslash\{i, j\}}\left(b_{k}^{v}-x_{k}\right)+b_{j}^{v}-x_{j} .
\end{aligned}
$$

Since $b_{j}^{v}>x_{j}$ and $b_{i}^{v}<x_{i}$, it holds that $m_{i j}^{v}\left(b^{v}, x\right)<m_{j i}^{v}\left(b^{v}, x\right)$ contradicting with the equal maximal complaint property. Therefore, claim 2 does not hold. By the above claims, $b_{i}^{v} \geq x_{i}, \forall i \in N$. 
Theorem 6.15. The optimal optimistic compromise value is the unique value satisfying efficiency and the equal optimistic maximal complaint property.

Proof. It is trivial that the optimal optimistic compromise value is efficient. By Proposition 6.1, $x_{i} \leq b_{i}^{v}, i \in N$, thus,

$$
\begin{aligned}
& m_{i j}^{v}\left(b^{v}, x\right)=b^{v}(N \backslash\{j\})-x(N \backslash\{j\})=\frac{n-1}{n}\left[b^{v}(N)-v(N)\right], \\
& m_{j i}^{v}\left(b^{v}, x\right)=b^{v}(N \backslash\{i\})-x(N \backslash\{i\})=\frac{n-1}{n}\left[b^{v}(N)-v(N)\right] .
\end{aligned}
$$

Therefore, $m_{i j}^{v}\left(b^{v}, x\right)=m_{j i}^{v}\left(b^{v}, x\right)$, i.e., the optimal optimistic compromise value satisfies the equal optimistic maximal complaint property.

It remains to prove the uniqueness part. Suppose that $x$ is a value with the two mentioned properties under the optimistic complaint criterion. For all $i \in N$, it holds that $x_{i} \leq b_{i}^{v}$ by Lemma 6.14 , thus,

$$
m_{i j}^{v}\left(b^{v}, x\right)=b^{v}(N \backslash\{j\})-x(N \backslash\{j\}) .
$$

Similarly,

$$
m_{j i}^{v}\left(b^{v}, x(v)\right)=b^{v}(N \backslash\{i\})-x(N \backslash\{i\}) .
$$

By the equal optimistic maximal complaint property, it holds $b^{v}(N \backslash\{i\})-$ $x(N \backslash\{i\})=b^{v}(N \backslash\{j\})-x(N \backslash\{j\})$ yielding the significant equation $b_{i}^{v}-x_{i}=$ $b_{j}^{v}-x_{j}$ for any $i, j \in N$. Therefore, it is easy to obtain $x_{i}=b_{i}^{v}-\frac{b^{v}(N)-v(N)}{n}$ by applying efficiency to the systems.

\subsubsection{Characterization of the optimal pessimistic compromise value}

Definition 6.10. For any $v \in \Gamma_{2}$ and $x \in I^{*}(v)$, the pessimistic maximal complaint $m_{i j}^{v}(x)$ of player $i \in N$ over another player $j \in N$ at payoff vector $x$ is given by the maximal pessimistic complaint among coalitions containing 
player $i$, but not containing player $j$ i.e.,

$$
m_{i j}^{v}(v(\cdot), x)=\max [e(S, v(\{\cdot\}), x) \mid S \subseteq N, i \in S, j \notin S] .
$$

A value $\varphi: \Gamma_{2} \rightarrow \mathbf{R}^{n}$ satisfies

- equal pessimistic maximal complaint property: if

$$
m_{i j}^{v}(v(\cdot), \varphi(v))=m_{j i}^{v}(v(\cdot), \varphi(v)), \text { for any } v \in \Gamma_{2}, i, j \in N,
$$

Lemma 6.16. For any $v \in \Gamma_{2}$, if $x$ is a payoff vector satisfying efficiency and the equal pessimistic maximal complaint property, it holds that $x_{i} \geq v(i), \forall i \in N$.

Proof. We prove the lemma by showing that the following two claims do not hold.

Claim 1: $x_{i}<v(i)$, for all $i \in N$.

Since $x_{i}<v(i), \forall i \in N$, thus,

$$
\sum_{i \in N} x_{i}<\sum_{i \in N} v(i)=v(N)
$$

which contradicts with $v \in \Gamma_{2}$. Therefore, claim 1 does not hold.

Claim 2: There exist two players $i, j \in N$, such that $x_{i}<v(i)$ and $x_{j}>v(j)$, $i, j \in N$.

By formula (6.22),

$$
\begin{aligned}
m_{i j}^{v}(v(\{\cdot\}), x) & =\max [e(S, v(\{\cdot\}), x) \mid S \subseteq N, i \in S, j \notin S] \\
& =\sum_{x_{k} \geq v(k), k \in N \backslash\{i, j\}}\left(x_{k}-v(k)\right)+x_{i}-v(i), \\
m_{j i}^{v}(v(\{\cdot\}), x) & =\max [e(S, v(\{\cdot\}), x) \mid S \subseteq N, j \in S, i \notin S] \\
& =\sum_{x_{k} \geq v(k), k \in N \backslash\{i, j\}}\left(x_{k}-v(k)\right)+x_{j}-v(j) .
\end{aligned}
$$


Since $x_{j}>v(j)$ and $x_{i}<v(i)$, it holds that $m_{i j}^{v}(v(\{\cdot\}), x)<m_{j i}^{v}(v(\{\cdot\}), x)$ contradicting with equal pessimistic maximal complaint property. Therefore, claim 2 does not hold.

By the above claims, $x_{i} \geq v(i), \forall i \in N$.

Theorem 6.17. The optimal pessimistic compromise value is the unique value with efficiency and equal pessimistic maximal complaint property.

Proof. It is trivial that the pessimistic optimal compromise value is efficient. By Proposition 6.2, $x_{i} \geq v(i), \forall i \in N$. Thus,

$$
\begin{aligned}
& m_{i j}^{v}\left(v(\{\cdot\}, x)=x(N \backslash\{j\})-\sum_{k \in N \backslash\{j\}} v(k)=\frac{n-1}{n}\left[v(N)-\sum_{k \in N} v(k)\right],\right. \\
& m_{j i}^{v}\left(v(\{\cdot\}, x)=x(N \backslash\{i\})-\sum_{k \in N \backslash\{i\}} v(k)=\frac{n-1}{n}\left[v(N)-\sum_{k \in N} v(k)\right] .\right.
\end{aligned}
$$

Therefore, $m_{i j}^{v}\left(b^{v}, x\right)=m_{j i}^{v}\left(b^{v}, x\right)$, i.e., the optimal pessimistic compromise value satisfies equal pessimistic maximal complaint property.

It remains to prove the uniqueness part. Suppose that $x$ is a value with the two mentioned properties. For any players $i \in N, x_{i} \geq v(i)$ by lemma 6.16 , we then have

$$
m_{i j}^{v}(v(\{\cdot\}), x)=x(N \backslash\{j\})-\sum_{k \in N \backslash\{j\}} v(k) .
$$

Similarly,

$$
m_{j i}^{v}(v(\{\cdot\}), x)=x(N \backslash\{i\})-\sum_{k \in N \backslash\{i\}} v(k) .
$$

By equal pessimistic maximal complaint property, it holds that $x(N \backslash\{i\})-$ $\sum_{k \in N \backslash\{i\}} v(k)=x(N \backslash\{j\})-\sum_{k \in N \backslash\{j\}} v(k)$ yielding the significant equation $x_{i}-$ $v(i)=x_{j}-v(j)$ for any $i, j \in N$. Therefore, it is easily to obtain $x_{i}=v(i)-$ $v(N)-\sum v(k)$

$\frac{k \in N}{n}$ by applying efficiency to the systems. 
The uniqueness part of Theorem 6.17 can also be proved by considering the anti-dual game $v^{\star}$.

Alternative Proof of Theorem 6.17. Let $\varphi$ be a value for game $v \in \Gamma_{2}$ satisfying efficiency and the equal pessimistic maximal complaint property. Let $v^{\star}$ be the anti-dual of game $v$ and construct $\phi=-\varphi$. Then it is easy to obtain $\phi(N)=-\varphi(N)=-v(N)=v^{*}(N)$, i.e., $\phi$ satisfies the efficiency for game $v^{\star}$. Next we prove that for $v^{\star}$, the equal optimistic maximal complaint property holds for $\phi$.

Clearly, by equal pessimistic maximal complaint property, $m_{i j}^{v}(v(\{\cdot\}), \varphi)=$ $m_{j i}^{v}(v(\{\cdot\}), \varphi)$. Therefore,

$$
\begin{aligned}
& \max [e(S, v(\{\cdot\}), \varphi) \mid S \subseteq N, i \in S, j \notin S]=\max [e(S, v(\{\cdot\}), \varphi) \mid S \subseteq N, j \in \\
& S, i \notin S] \\
& \Leftrightarrow \max \left[\varphi(N \backslash S)-\sum_{k \in N \backslash S} v(k) \mid i \in S, j \notin S\right]=\max \left[\varphi(N \backslash S)-\sum_{l \in N \backslash S} v(l) \mid\right. \\
& j \in S, i \notin S] \\
& \Leftrightarrow \max \left[-\phi(N \backslash S)+\sum_{k \in N \backslash S} b_{k}^{v^{*}} \mid i \in S, j \notin S\right]=\max \left[-\phi(N \backslash S)+\sum_{l \in N \backslash S} b_{l}^{v^{*}} \mid\right. \\
& \Leftrightarrow m_{i j}^{v^{*}}\left(b^{v^{\star}}, \phi\right)=m_{j i}^{v^{*}}\left(b^{v^{*}}, \phi\right),
\end{aligned}
$$

which yields $\phi$ satisfies equal optimistic maximal complaint property. Hence, by Theorem 6.15, $\phi$ is the optimal optimistic compromise value for game $\left(N, v^{\star}\right)$. That is, $\phi_{i}=\operatorname{ENSC}_{i}\left(N, v^{\star}\right)=b_{i}^{v^{\star}}+\frac{1}{n}\left[v^{\star}(N)-\sum_{k \in N} b_{k}^{v^{\star}}\right]$. Note that $\phi_{i}=-\varphi_{i}, v^{\star}(N)=-v(N)$ and $b_{i}^{v^{\star}}=-v(i)$, therefore $\varphi$ is uniquely determined and $\varphi_{i}=v(i)+\frac{1}{n}\left[v(N)-\sum_{k \in N} v(k)\right], i \in N$.

Remark 6.2. Actually the optimal optimistic (pessimistic) average compromise value can also be characterized in the same manner. First we should define the complaint for one player over other players in an average way as follows. 
Definition 6.11. For any game $(N, v) \in \Gamma_{1}$, given the optimistic average complaint criterion, the optimistic maximal average complaint $h_{i j}^{v}\left(x, b^{v}\right)$ of player $i \in N$ against another player $j \in N$ with respect to payoff vector $x$ is defined as the maximal per capita complaint among coalitions containing player $i$, but not containing player $j$, i.e.,

$$
h_{i j}^{v}\left(x, b^{v}\right)=\max \left[\bar{e}\left(S, x, b^{v}\right) \mid S \subseteq N, i \in S, j \notin S\right] .
$$

Definition 6.12. For any game $(N, v) \in \Gamma_{2}$, given the pessimistic average complaint criterion, the pessimistic maximal average complaint $h_{i j}^{v}(x, v(\{\cdot\}))$ of player $i \in N$ against another player $j \in N$ with respect to payoff vector $x$ is defined as the maximal per capita complaint among coalitions containing player $i$, but not containing player $j$, i.e.,

$$
h_{i j}^{v}(x, v(\{\cdot\}))=\max [\bar{e}(S, x, v(\{\cdot\})) \mid S \subseteq N, i \in S, j \notin S] .
$$

Given the optimistic or pessimistic optimal average compromise value, players all have the same complaint to others with respect to the above maximal complaints respectively, which are called equal optimistic (pessimistic) maximal average complaint property. Without going into details, we provide the characterization of these two average compromise values, of which the proofs are inspired by that of Theorem 6.15 and Theorem 6.17.

Theorem 6.18. For any game $(N, v) \in \Gamma_{1}$, the optimal optimistic average complaint value is the unique value satisfying efficiency and the equal optimistic maximal average complaint property.

Theorem 6.19. For any game $(N, v) \in \Gamma_{2}$, the optimal pessimistic average complaint value is the unique value satisfying efficiency and the equal pessimistic maximal average complaint property.

As a conclusion of this chapter, we generalize the results by briefly introducing two other optimal compromise values, namely the $\alpha$-ENSC value and the $\alpha$-CIS value. To do that, the $\alpha$-optimistic complaint and $\alpha$-pessimistic 
complaint are proposed such that the coalition S's complaints are given by

$$
\begin{aligned}
& e\left(S, x, \alpha b^{v}\right)=\alpha b^{v}(S)-x(S), \\
& e(S, x, \alpha v(\{\cdot\}))=x(N \backslash S)-\alpha \sum_{j \in N \backslash S} v(j) .
\end{aligned}
$$

The corresponding $\alpha$-optimistic and $\alpha$-pessimistic maximal complaints are defined by

$$
\begin{aligned}
& m_{i j}^{v}\left(\alpha b^{v}, x\right)=\max \left[\alpha b^{v}(S)-x(S) \mid S \subseteq N, i \in S, j \notin S\right], \\
& m_{i j}^{v}(\alpha v(\cdot), x)=\max \left[x(N \backslash S)-\alpha \sum_{k \in N \backslash S} v(k) \mid S \subseteq N, i \in S, j \notin S\right] .
\end{aligned}
$$

Then we have the following result

(i) The $\alpha$-ENSC value is the unique value satisfying efficiency and the equal $\alpha$-optimistic maximal complaint property.

(ii) The $\alpha$-CIS value is the unique value satisfying efficiency and the equal $\alpha$-pessimistic maximal complaint property. 


\section{Chapter 7}

\section{Optimal solution concepts for cooperative games with stochastic payoffs}

In this chapter, we explore solution concepts for cooperative games with stochastic payoffs. First, by minimizing the total variance of excesses of all coalitions, we define the set of most stable solutions, i.e., the solutions that allocate the risk equally among players. Next we define the fairest solution as the one for which the excesses of coalitions are closest to the average excess. As it turns out, there is a unique such solution and this happens to be one of the most stable ones as well.

\subsection{Introduction}

The classical (deterministic) cooperative game theory provides practical tools to model situations with cooperation. The main issue is to find fair allocation schemes of the total societal benefit generated by the participants. In many cases payoffs to coalitions are uncertain, e.g., linear production games with uncertain prices [78], sequencing games [18] with uncertain processing and ready time [77], bankruptcy games [4,59] with stochastic estates and 
claims [35] and inventory centralization games [60] with stochastic random demand [87]. Uncertainty in these situations is problematic, in particular if the allocation rule is to be fixed before the realization of the coalitions' payoffs.

The extension of the theory of cooperative games in characteristic function form into stochastic situations can be found in [13] and [15]. They both considered situations in which payoffs to coalitions are stochastic variables. A series of two stage solutions was investigated by Charnes et al. $[14,15]$. In the first stage, players are promised some most realistic payoffs, which are more likely to achieve, called prior payoffs. In the second stage, once the stochastic payoffs are observed, modification of the prior payoff will be carried out in case it is impossible to keep the initial promise. As an alternative to Charnes and Granot's method [14,15], Suijs et al. [76,77] introduced preferences to the model and proposed the concept of core of the stochastic cooperative games . Other solution concepts can also be found in literature, e.g. Fernandez et al. [28] introduced cores of stochastic cooperative games by introducing stochastic orders. Other researchers also investigated different concepts of cores, such as the sequential core [100], strong sequential cores $[63,86]$, weak sequential core [34], and also cores for incomplete market economies [33]. In fact, most of the work referred to above has developed concepts for stochastic games that do not apply translations into a deterministic equivalent.

In this chapter, we explore solution concepts defined as the optimal solutions of corresponding optimization problems involving the excesses of coalitions and individual players, based on both the least square and lexicographic criterion. Primarily, the application of the least square criterion in this paper is inspired by the work of Ruiz et al. [68-70]. They introduced the well-known family of least square values as the unique (pre)imputation that minimizes the variance of the excesses of the coalitions. Recall that the concept of excess is generally regarded as the measurement index of the dissatisfaction of coalitions at the potential payoff vectors for deterministic cooperative games. Inspired by their work, we explore the optimal allocations also based on the idea of excess. In deterministic situations, all decision-making information is 
perfect, while when it comes to stochastic situations, the most prominent difficulty is how to deal with uncertainty. We first construct optimization problems that minimize the sum of variances of all coalitions' excesses among the allocation set. In this way, an interesting solution concept emerges. The set of most stable solutions, as we call it, allocates the risk part of the payoff equally among all players. However, a significant drawback of the most stable solutions is its non-uniqueness, which is obviously not a convincing choice in decision-making situations. To solve this problem, we instead minimize the total expected excesses of all coalitions under the least square criterion. It turns out that this results in a unique optimal solution. Interestingly, the optimal solution, which we call the fairest solution, belongs to the set of most stable solutions. Moreover, these two solutions can also be obtained as the optimal solutions of optimization problems involving the excess from the perspective of individual players based on the marginal contributions.

\subsection{Preliminaries}

Charnes and Granot [13] first introduced the definition of cooperative game with stochastic payoffs.

Definition 7.1. A cooperative game with stochastic payoffs in characteristic function form is a pair $(N, \mathscr{V})$, where $\mathscr{V}: 2^{N} \rightarrow L^{1}(\mathbf{R})$ is the characteristic function which assigns to each coalition $S$ of $N$ a stochastic variable $\mathscr{V}(S) \in$ $L^{1}(\mathbf{R})$, which is the set of one-dimensional stochastic variables.

As usual, $\mathscr{V}(S)$ is interpreted as the payoff a coalition can achieve by acting alone, irrespective of the actions of the players outside the coalition. Such a consideration may lead to the conclusion that the stochastic worths of all coalitions are independent. However, even under the usual interpretation of the worth of a coalition, independence of worths does not follow and is in fact a strong assumption. For instance, in many situations where the worth of $\mathscr{V}(S)$ has low realization, one would expect low realizations for subcoalitions of $S$ as well. Seminal work on the topic, like Charnes and Granot [14], makes a similar independence assumption. However, more recent work, like Habis and Herings [34], allows for arbitrary dependencies between 
the stochastic variables that specify the worths of coalitions. In our work, we only require the independences between the stochastic worth of non-grand coalitions $(S \neq N)$ and the grand coalition $N$. In the remainder of this chapter, we also assume that the worths of all coalitions are real valued stochastic variables with finite expectations and variances. Denote the set of all these cooperative games with stochastic payoffs and player set $N$ by $S G(N)$. The expectation of a stochastic variable $\xi \in L^{1}(\mathbf{R})$ is denoted by $\mathbf{E}[\xi]$ and the variance of $\xi$ is $\mathbf{D}[\xi]$. Moreover, the covariance between two stochastic variables $\xi, \eta \in L^{1}(\mathbf{R})$ is $\operatorname{cov}(\xi, \eta)$.

As for deterministic cooperative games, the main issue is to determine a fair allocation of the total stochastic worth $\mathscr{V}(N)$ of the grand coalition. Allocations for players must of course be stochastic variables, too. Suijs et al. [76] proposed the following allocation form.

Definition 7.2. For any $(N, \mathscr{V}) \in S G(N)$, an allocation for this game is defined as a pair $(d, r) \in \mathbf{R}^{n} \times \mathbf{R}^{n}$ satisfying:

(i) $d(N)=\sum_{i \in N} d_{i}=\mathbf{E}[\mathscr{V}(N)]$;

(ii) $r(N)=\sum_{i \in N} r_{i}=1$ and $r_{i} \geq 0$ for all $i \in N$.

The payoff to player $i$ with respect to $(d, r)$ is defined as

$$
x_{i}=(d, r)_{i}:=d_{i}+r_{i}\{\mathscr{V}(N)-\mathbf{E}[\mathscr{V}(N)]\} .
$$

Notice that payoffs to players under this model consist of two parts: $d_{i}$ is the allocation of the expectation $\mathbf{E}[\mathscr{V}(N)]$ of the grand coalition and $r_{i}$ is the allocation of the residual $\mathscr{V}(N)-\mathbf{E}[\mathscr{V}(N)]$, which is also interpreted as the risk of the stochastic variable $\mathscr{V}(N)$. Let $D R(\mathscr{V})$ denote the set of all allocations of this type for any game $(N, \mathscr{V}) \in S G(N)$.

For any $x \in D R(\mathscr{V})$ and $S \subseteq N$, write $e^{\mathscr{V}}(S, x):=\mathscr{V}(S)-x(S)$, where $x(S)=\sum_{i \in S} x_{i}$. As in deterministic cooperative games, we call $e^{\mathscr{V}}(S, x)$ the excess of coalition $S$ with respect to allocation $x$. The concept of excess is generally regarded as the dissatisfaction of coalitions. In fact, it can also be applied to the stochastic case. The difference is that the excess $e^{\mathscr{V}}(S, x)$ is a stochastic variable with known distribution function. If there is no confusion, we will write $e(S, x)$ instead of $e^{y}(S, x)$ throughout the paper. In the next 
sections, we will focus on the selection of allocations from the set $D R(\mathscr{V})$ using various optimization models.

\subsection{Optimization models for stochastic cooperative games based on coalitions}

Recall that Ruiz et al. [68] introduced the least square (pre)nucleolus that minimizes the total variance of all coalitions among the (pre)imputation set under the least square criterion. Different from the discrete cases Ruiz et al. have discussed, we concentrate on optimization models for stochastic situations, which are continuous. The section is split into two subsections. The concepts of the most stable solutions and the fairest solutions are defined as the optimal solutions of corresponding optimization problems respectively.

\subsubsection{The most stable solutions for stochastic cooperative games}

In probability theory and statistics, variance is the expectation of the squared deviation of a stochastic variable from its mean, and it generally measures how far a set of (stochastic) numbers are spread out from their mean. When making decisions in stochastic environment, the main concern is the stability of the outcome. Thus we first consider the following problem which minimizes the variances of excesses of all coalitions over allocation set $D R(\mathscr{V})$.

\section{Problem 7.1.}

$$
\begin{aligned}
& \min \sum_{S \subseteq N} \mathbf{D}[e(S, x)] \\
& \text { s.t. } \quad x \in D R(\mathscr{V}) .
\end{aligned}
$$

To explore the optimal solutions of Problem 7.1, it will be useful to simplify the objective function of this problem. Denote the objective function of 
Problem 7.1 as $P_{1}$. Then we have

$$
\begin{aligned}
P_{1} & =\sum_{S \subset N} \mathbf{D}[\mathscr{V}(S)-x(S)] \\
& =\sum_{S \subset N} \mathbf{D}[\mathscr{V}(S)-d(S)-r(S) \mathscr{V}(N)+r(S) \mathbf{E}[\mathscr{V}(N)]] \\
& =\sum_{S \subset N} \mathbf{D}[\mathscr{V}(S)-r(S) \mathscr{V}(N)] \\
& =\sum_{S \subset N}\left\{\mathbf{D}[\mathscr{V}(S)]+r^{2}(S) \mathbf{D}[\mathscr{V}(N)]-2 r(S) \operatorname{cov}(\mathscr{V}(S), \mathscr{V}(N))\right\} \\
& =\sum_{S \subset N}\left\{\mathbf{D}[\mathscr{V}(S)]+r^{2}(S) \mathbf{D}[\mathscr{V}(N)]\right\} .
\end{aligned}
$$

The last equation is derived from the assumption that $\mathscr{V}(S)$ is independent of $\mathscr{V}(N)$. The objection function $P_{1}$ does not contain the unknown quantity $d$, thus we can restrict our attention to $r$. That is, Problem 7.1 can be simplified into the following problem:

Problem 7.2.

$$
\begin{aligned}
& \min \sum_{S \subseteq N} r^{2}(S) \mathbf{D}[\mathscr{V}(N)] \\
& \text { s.t. } \quad r(N)=1, r_{i} \geq 0, \forall i \in N .
\end{aligned}
$$

In case the variance of the grand coalition is zero, which will degenerate into the deterministic cooperative game, then the optimal solution turns out to be the whole feasible area $D R(\mathscr{V})$. So it is reasonable to assume that the variance of the grand coalition is positive, that is, $\mathscr{V}(N)$ is not a constant.

Theorem 7.1. For any $(N, \mathscr{V}) \in S G(N)$, the solutions of Problem 7.1 are as follows:

$$
x_{i}^{*}=d_{i}^{*}+r_{i}^{*}(\mathscr{V}(N)-E[\mathscr{V}(N)]),
$$

where $r_{i}^{*}=\frac{1}{n}, \forall i \in N$ and $d^{*}$ satisfies $\sum_{i \in N} d_{i}^{*}=E[\mathscr{V}(N)]$.

Proof. By calculating the Hessian matrix, it can easily be checked that the objective function of Problem 7.2 is strictly convex, and so is the feasible set. Hence, there exists at most one optimal solution of Problem 7.2. It remains 
to verify the Lagrange conditions. The Lagrange function of Problem 7.2 is

$$
L(r, \lambda)=\sum_{S \subseteq N} r^{2}(S) \mathbf{D}[\mathscr{V}(N)]+\lambda(r(N)-1) .
$$

Taking the partial derivative with respect to $r_{i}$ in (7.3), we obtain the Lagrangian conditions

$$
L_{i}(r, \lambda)=2 \sum_{S \ni i} r(S) \mathbf{D}[\mathscr{V}(N)]+\lambda=0, \forall i \in N .
$$

Together with the constraint condition, it is not difficult to obtain that the solution of Problem 7.2 is $r_{i}^{*}=\frac{1}{n}$ for all $i \in N$. In conclusion, the solutions of Problem 7.1 is given by

$$
x_{i}^{*}=d_{i}^{*}+\frac{1}{n}\{\mathscr{V}(N)-\mathbf{E}[\mathscr{V}(N)]\}, \forall i \in N,
$$

where $d^{*}$ satisfies $\sum_{i \in N} d_{i}^{*}=\mathbf{E}[\mathscr{V}(N)]$.

As mentioned earlier, the concept of variance reflects the amount of dispersion of samples to their mean. A larger variance implies more uncertainty of the stochastic variable. By minimizing the total dispersion of the excess of all coalitions, we can see that the optimal solution of Problem 7.1 distributes the uncertainty part (namely the risk part), $\mathscr{V}(N)-\mathbf{E}[\mathscr{V}(N)]$ equally among the players. Formally, we define such allocation rule as the so-called most stable allocations for stochastic cooperative games.

Definition 7.3. For any $(N, \mathscr{V}) \in S G(N)$, the most stable solutions for $(N, \mathscr{V})$, denoted by $M S(\mathscr{V})$, are the optimal solutions of Problem 7.1.

One possible choice for the $d_{i}^{*}$ in (7.2) would be to take our favorite solution concept for classical games and apply it to the corresponding expectation game $\left(N, \mathscr{V}^{E}\right)$, defined by $\mathscr{V}^{E}(S)=\mathrm{E}[\mathscr{V}(S)]$ for all $S \subseteq N$. Obviously, $\left(N, \mathscr{V}^{E}\right)$ is a deterministic cooperative game. Moreover, for any $x=(d, r) \in D R(\mathscr{V})$, it turns out that the condition $\sum_{i \in N} d_{i}^{*}=\mathrm{E}[\mathscr{V}(N)]$ is equivalent to $d \in I^{*}\left(\mathscr{V}^{E}\right)=$ $\left\{x \in \mathbf{R}^{n} \mid x(N)=\mathscr{V}^{E}(N)\right\}$. Hence we could select the classical solutions for game $\left(N, \mathscr{V}^{E}\right)$ as the allocation of $d$, e.g. the Shapley value [72], core [29], 
Chapter 7. Optimal solution concepts for cooperative games with stochastic payoffs

kernel [19], the solidarity value [57] and the nucleolus [71]. Further discussion about the determination of $d$ will be shown in the next sections.

\subsubsection{The fairest solution for stochastic cooperative games}

Notice that the most stable solutions merely determine the allocation of the stochastic part, i.e., $\mathscr{V}(N)-\mathbf{E}[\mathscr{V}(N)]$, but ignore the distribution of the deterministic part $\mathbf{E}[\mathscr{V}(N)]$. A natural question is whether we could select a unique allocation from the most stable solution set. To this end we consider

\section{Problem 7.3.}

$$
\begin{aligned}
& \min \sum_{S \subseteq N} \mathbf{E}\left[\{e(S, x)-\bar{e}(\mathscr{V}, x)\}^{2}\right] \\
& \text { s.t. } \quad x \in D R(\mathscr{V}),
\end{aligned}
$$

where $\bar{e}(\mathscr{V}, x)=\frac{1}{2^{n}-1} \sum_{S \subseteq N} e(S, x)$ is the average excess over all nonempty coalitions at allocation $x$. If there exists an optimal solution of Problem 7.3, then it is the unique allocation for which the excesses of coalitions are closest to the average excess, thus the optimization model in some sense implements the egalitarian principle towards the coalitions. In fact, the average excess is the same for all allocations in $D R(\mathscr{V})$. Similar to Lemma 4 in [68], we present the following

Proposition 7.1. For any $(N, \mathscr{V}) \in S G(N)$, the sum of all the excesses of all coalitions is the same at all $x \in D R(\mathscr{V})$.

Proof. Given a $x \in D R(\mathscr{V})$, then we have

$$
\begin{aligned}
& \sum_{S \subseteq N} e(S, x)=\sum_{S \subseteq N}\{\mathscr{V}(S)-x(S)\} \\
& =\sum_{S \subseteq N}\{\mathscr{V}(S)-d(S)-r(S) \mathscr{V}(N)+r(S) \mathbf{E}[\mathscr{V}(N)]\} \\
& =\sum_{S \subseteq N} \mathscr{V}(S)-\sum_{S \subseteq N} d(S)-\sum_{S \subseteq N} r(S) \mathscr{V}(N)+\sum_{S \subseteq N} r(S) \mathbf{E}[\mathscr{V}(N)] \\
& =\sum_{S \subseteq N} \mathscr{V}(S)-2^{n-1} d(N)-2^{n-1} \mathscr{V}(N)+2^{n-1} \mathbf{E}[\mathscr{V}(N)]
\end{aligned}
$$




$$
=\sum_{S \subseteq N} \mathscr{V}(S)-2^{n-1} \mathscr{V}(N)
$$

The last equation results from the fact that $\sum_{i \in N} d_{i}=\mathbf{E}[\mathscr{V}(N)]$.

According to Proposition 7.1, the average excess is irrelevant to the allocation, hence throughout the paper we write $\bar{e}(\mathscr{V})$ rather than $\bar{e}(\mathscr{V}, x)$. The next theorem reveals the existence and uniqueness of the solution of Problem 7.3.

Theorem 7.2. For any $(N, \mathscr{V}) \in S G(N)$, there exists a unique solution $x^{*}$ of Problem 7.3, i.e.,

$$
x_{i}^{*}=d_{i}^{*}+r_{i}^{*}\{\mathscr{V}(N)-E[\mathscr{V}(N)]\},
$$

where $r_{i}^{*}=\frac{1}{n}, \forall i \in N$, and

$$
d_{i}^{*}=\frac{1}{n} E[\mathscr{V}(N)]+\frac{1}{2^{n-2}}\left[e_{i}(\mathscr{V})-\frac{1}{n} \sum_{j \in N} e_{j}(\mathscr{V})\right], \forall i \in N,
$$

where $e_{i}(\mathscr{V})=\sum_{S \ni i} E[\mathscr{V}(S)]$.

In order to prove Theorem 7.2, we need the following lemma.

Lemma 7.3. For any $(N, \mathscr{V}) \in S G(N)$, the solution of Problem 7.3 coincides with the solution of the following problem:

\section{Problem 7.4.}

$$
\begin{aligned}
& \min \widetilde{P_{2}} \\
& \text { s.t. } \quad x \in D R(\mathscr{V}),
\end{aligned}
$$

where

$$
\begin{aligned}
\widetilde{P}_{2} & =\sum_{S \subseteq N}\left\{d^{2}(S)+\left(\frac{2}{2^{n}-1} \sum_{T \subseteq N} \mathbf{E}[\mathscr{V}(T)]-2 \mathbf{E}[\mathscr{V}(S)]-\frac{2^{n}}{2^{n}-1} \mathbf{E}[\mathscr{V}(N)]\right) d(S)\right. \\
& \left.+\mathbf{D}[\mathscr{V}(N)] r^{2}(S)-\frac{2^{n}-2}{2^{n}-1} \mathbf{D}[\mathscr{V}(N)] r(S)\right\}
\end{aligned}
$$


Chapter 7. Optimal solution concepts for cooperative games with stochastic payoffs

Proof. Denote the objective function of Problem 7.3 as $P_{2}$. Then we have

$$
\begin{aligned}
P_{2} & =\sum_{S \subseteq N} \mathbf{E}\left[\left\{(\mathscr{V}(S)-x(S))^{2}+\bar{e}(\mathscr{V})^{2}-2(\mathscr{V}(S)-x(S)) \bar{e}(\mathscr{V})\right\}\right] \\
& =\underbrace{\sum_{S \subseteq N} \mathbf{E}\left[\left\{(\mathscr{V}(S)-x(S)\}^{2}\right]\right.}_{P_{21}}+\underbrace{\sum_{S \subseteq N} \mathbf{E}\left[\bar{e}(\mathscr{V})^{2}\right]}_{P_{22}}-\underbrace{2 \sum_{S \subseteq N} \mathbf{E}[(\mathscr{V}(S)-x(S)) \bar{e}(\mathscr{V})]}_{P_{23}} \\
& =P_{21}+P_{22}+P_{23} .
\end{aligned}
$$

Next we will analyze these three parts.

$$
\begin{aligned}
P_{21} & =\sum_{S \subseteq N} \mathbf{E}\left[\left\{(\mathscr{V}(S)-x(S)\}^{2}\right]\right. \\
& =\sum_{S \subseteq N} \mathbf{E}\left[\{\mathscr{V}(S)-d(S)-r(S) \mathscr{V}(N)+r(S) \mathbf{E}[\mathscr{V}(N)]\}^{2}\right] \\
& =\sum_{S \subseteq N} \mathbf{E}\left[(\mathscr{V}(S)-d(S))^{2}+r^{2}(S) \mathbf{D}[\mathscr{V}(N)]\right. \\
& -2 r(S)(\mathscr{V}(S)-d(S))(\mathscr{V}(N)-\mathbf{E}[\mathscr{V}(N)])] \\
& =\sum_{S \subseteq N}\left(\mathbf{E}\left[\mathscr{V}^{2}(S)\right]-2 d(S) \mathbf{E}[\mathscr{V}(S)]+d^{2}(S)+r^{2}(S) \mathbf{D}[\mathscr{V}(N)]\right) .
\end{aligned}
$$

According to Proposition 7.1, the average excess $\bar{e}(\mathscr{V})$ is actually irrelevant to the allocation $x$, that is, it contains no unknown quantities $d$ and $r$. Therefore eliminating the item $P_{22}$ from $P_{2}$ does not affect the optimal solution of Problem 7.3. As to the last item $P_{23}$, we have

$$
\begin{aligned}
P_{23} & =2 \sum_{S \subseteq N} \mathrm{E}[(\mathscr{V}(S)-x(S)) \bar{e}(\mathscr{V})] \\
& \left.=2 \sum_{S \subseteq N} \mathrm{E}[(\mathscr{V}(S)-x(S)])\left(\frac{1}{2^{n}-1} \sum_{T \subseteq N} \mathscr{V}(T)-\frac{2^{n-1}}{2^{n}-1} \mathscr{V}(N)\right)\right] \\
& \left.=\frac{2}{2^{n}-1} \sum_{S \subseteq N} \mathrm{E}\left[\sum_{T \subseteq N} \mathscr{V}(T)(\mathscr{V}(S)-x(S)]\right)\right] \\
& \left.-\frac{2^{n}}{2^{n}-1} \sum_{S \subseteq N} \mathrm{E}[(\mathscr{V}(S)-x(S)]) \mathscr{V}(N)\right]
\end{aligned}
$$




$$
\begin{aligned}
& =\frac{2}{2^{n}-1} \underbrace{\sum_{S \subseteq N} \mathrm{E}\left[\sum_{T \subseteq N} \mathscr{V}(T)(\mathscr{V}(S)-d(S)-r(S) \mathscr{V}(N)+r(S) \mathrm{E}[\mathscr{V}(N)])\right]}_{P_{23}^{1}} \\
& -\frac{2^{n}}{2^{n}-1} \underbrace{\sum_{S \subseteq N} \mathrm{E}[(\mathscr{V}(S)-d(S)-r(S) \mathscr{V}(N)+r(S) \mathrm{E}[\mathscr{V}(N)]) \mathscr{V}(N)]}_{P_{23}^{2}} \\
& =\frac{2}{2^{n}-1} P_{23}^{1}-\frac{2^{n}}{2^{n}-1} P_{23}^{2} .
\end{aligned}
$$

Furthermore, the first item $P_{23}^{1}$ can be rewritten as follows:

$$
\begin{aligned}
P_{23}^{1} & =\sum_{S \subseteq N} \mathbf{E}\left[\sum_{T \neq S, N} \mathscr{V}(T)(\mathscr{V}(S)-d(S)-r(S) \mathscr{V}(N)+r(S) \mathbf{E}[\mathscr{V}(N)])\right] \\
& +\sum_{S \subseteq N} \mathbf{E}\left[\mathscr{V}^{2}(S)-d(S) \mathscr{V}(S)-r(S) \mathscr{V}(N) \mathscr{V}(S)+r(S) \mathbf{E}[\mathscr{V}(N)] \mathscr{V}(S)\right] \\
& +\sum_{S \subseteq N} \mathbf{E}\left[\mathscr{V}(S) \mathscr{V}(N)-d(S) \mathscr{V}(N)-r(S) \mathscr{V}^{2}(N)+r(S) \mathbf{E}[\mathscr{V}(N)] \mathscr{V}(N)\right] \\
& =\sum_{S \subseteq N} \mathbf{E}\left[\sum_{T \neq S, N} \mathscr{V}(T)(\mathscr{V}(S)-d(S))\right]+\sum_{S \subseteq N} \mathbf{E}\left[\mathscr{V}^{2}(S)-d(S) \mathscr{V}(S)\right] \\
& +\sum_{S \subseteq N}\{\mathbf{E}[\mathscr{V}(S) \mathscr{V}(N)]-d(S) \mathbf{E}[\mathscr{V}(N)]-r(S) \mathbf{D}[\mathscr{V}(N)]\}
\end{aligned}
$$

Observe that the last item of the last equation is equivalent to $P_{23}^{2}$. Thus we have

$$
\begin{aligned}
P_{23} & =\frac{2}{2^{n}-1} \sum_{S \subseteq N} \mathbf{E}\left[\sum_{T \neq S, N} \mathscr{V}(T)(\mathscr{V}(S)-d(S))\right] \\
& +\frac{2}{2^{n}-1} \sum_{S \subseteq N} \mathbf{E}\left[\mathscr{V}^{2}(S)-d(S) \mathscr{V}(S)\right] \\
& -\frac{2^{n}-2}{2^{n}-1} \sum_{S \subseteq N}\{\mathbf{E}[\mathscr{V}(S) \mathscr{V}(N)]-d(S) \mathbf{E}[\mathscr{V}(N)]-r(S) \mathbf{D}[\mathscr{V}(N)]\} \\
& =\frac{2}{2^{n}-1} \sum_{S \subseteq N}\left\{\sum_{T \neq S, N} \mathbf{E}[\mathscr{V}(T)](\mathbf{E}[\mathscr{V}(S)]-d(S))+\mathbf{E}\left[\mathscr{V}^{2}(S)\right]-d(S) \mathbf{E}[\mathscr{V}(S)]\right. \\
& \left.+\left(2^{n-1}-1\right) \mathbf{E}[\mathscr{V}(N)](d(S)-\mathbf{E}[\mathscr{V}(S)])+\left(2^{n-1}-1\right) r(S) \mathbf{D}[\mathscr{V}(N)]\right\}
\end{aligned}
$$




$$
\begin{aligned}
& =\frac{2}{2^{n}-1} \sum_{S \subseteq N}\left\{\sum_{T \subseteq N} \mathbf{E}[\mathscr{V}(T)](\mathbf{E}[\mathscr{V}(S)]-d(S))\right. \\
& \left.+2^{n-1} \mathbf{E}[\mathscr{V}(N)](d(S)-\mathbf{E}[\mathscr{V}(S)])+\left(2^{n-1}-1\right) r(S) \mathbf{D}[\mathscr{V}(N)]+\mathbf{D}[\mathscr{V}(S)]\right\}
\end{aligned}
$$

Note that the optimal solution of Problem 7.3 will not change by eliminating the constant items and corresponding positive coefficients in the objective function $P_{2}$. Denote the simplified objective function as $\widetilde{P_{2}}$, i.e.,

$$
\begin{aligned}
\widetilde{P}_{2} & =\sum_{S \subseteq N}\left\{\mathbf{D}[\mathscr{V}(N)] r^{2}(S)-\frac{2^{n}-2}{2^{n}-1} \mathbf{D}[\mathscr{V}(N)] r(S)\right. \\
& \left.+d^{2}(S)+\left(\frac{2}{2^{n}-1} \sum_{T \subseteq N} \mathbf{E}[\mathscr{V}(T)]-2 \mathbf{E}[\mathscr{V}(S)]-\frac{2^{n}}{2^{n}-1} \mathbf{E}[\mathscr{V}(N)]\right) d(S)\right\}
\end{aligned}
$$

Thus, indeed, Problem 7.3 is equivalent to Problem 7.4.

Proof of Theorem 7.2. Similar to the proof of Theorem 7.1, one can verify that there exists at most one optimal solution of Problem 7.4. Imposing the Lagrangian multiplication factors $\lambda, \omega$, we obtain the Lagrangian function of this problem

$$
L(x, \lambda, \omega)=\widetilde{P_{2}}+\lambda(d(N)-\mathbf{E}[\mathscr{V}(N)])+\omega(r(N)-1) .
$$

Taking the partial derivative with respect to $d_{i}$ in equation (7.9), the Lagrangian conditions for $d$ are

$$
\begin{aligned}
L_{d_{i}}(x, \lambda, \omega) & =\sum_{S \ni i}\left\{2 d(S)-2 \mathbf{E}[\mathscr{V}(S)]+\frac{2}{2^{n}-1} \sum_{T \subseteq N} \mathbf{E}[\mathscr{V}(T)]\right. \\
& \left.-\frac{2^{n}}{2^{n}-1} \mathbf{E}[\mathscr{V}(N)]\right\}+\lambda=0, \quad \forall i \in N .
\end{aligned}
$$

Denote

$$
\begin{aligned}
e_{i}(\mathscr{V}) & =\sum_{S \ni i} \mathrm{E}[\mathscr{V}(S)] \\
C(\mathscr{V}) & =\frac{2}{2^{n}-1} \sum_{T \subseteq N} \mathrm{E}[\mathscr{V}(T)]-\frac{2^{n}}{2^{n}-1} \mathbf{E}[\mathscr{V}(N)] .
\end{aligned}
$$


The Lagrangian conditions immediately give the following relations:

$2 \sum_{S \ni i} d(S)-2 e_{i}(\mathscr{V})+\sum_{S \ni i} C(\mathscr{V})=2 \sum_{S \ni j} d(S)-2 e_{j}(\mathscr{V})+\sum_{S \ni j} C(\mathscr{V}), \quad \forall i, j \in N$

Observe that $C(\mathscr{V})$ is a constant for any given game $(N, \mathscr{V}) \in S G(N)$, thus Equation (7.10) can be simplified as

$$
\sum_{S \ni i} d(S)-e_{i}(\mathscr{V})=\sum_{S \ni j} d(S)-e_{j}(\mathscr{V}), \quad \forall i, j \in N
$$

Together with the constraint condition $d(N)=\mathbf{E}[\mathscr{V}(N)]$, it is not difficult to solve this linear system. The unique optimal point has the form as follows:

$$
d_{i}^{*}=\frac{1}{n} \mathbf{E}[\mathscr{V}(N)]+\frac{1}{n 2^{n-2}}\left[n e_{i}(\mathscr{V})-\sum_{j \in N} e_{j}(\mathscr{V})\right], \quad \forall i \in N .
$$

Similarly, taking the partial derivative with respect to $r_{i}$ in (7.9), then the Lagrangian conditions with respect to $r_{i}$ are

$$
L_{r_{i}}(x, \lambda, \omega)=\sum_{S \ni i}\left\{2 \mathbf{D}[\mathscr{V}(N)] r(S)-\frac{2^{n}-2}{2^{n}-1} \mathbf{D}[\mathscr{V}(N)]\right\}+\omega=0,
$$

which is equivalent to the following conditions for all $i, j \in N$,

$$
\sum_{S \ni i}\left(2 r(S)-\frac{2^{n}-2}{2^{n}-1}\right) \mathbf{D}[\mathscr{V}(N)]=\sum_{S \ni j}\left(2 r(S)-\frac{2^{n}-2}{2^{n}-1}\right) \mathbf{D}[\mathscr{V}(N)] .
$$

Eliminating the constant items and positive coefficient gives the simplified equations as follows:

$$
\sum_{S \ni i} r(S)=\sum_{S \ni j} r(S), \quad \forall i, j \in N .
$$

Obviously, the optimal solution $r^{*}$ of this linear system is $r_{i}^{*}=\frac{1}{n}$, for all $i \in$ $N$. In summary, the allocation in Equation (7.14) is the optimal solution of Problem 7.4 as well as Problem 7.3.

As it turns out, the optimal solution of Problem 7.3 belongs to the set of 
the most stable solutions $M S(\mathscr{V})$, and it also determines the distribution of the expectation of $\mathscr{V}(N)$. Furthermore, note that the optimum solution $d^{*}$ satisfies Equation (7.11), which can be rewritten as

$$
\sum_{S \ni i}\left(\mathbf{E}[\mathscr{V}(S)]-d^{*}(S)\right)=\sum_{S \ni j}\left(\mathbf{E}[\mathscr{V}(S)]-d^{*}(S)\right), \quad \forall i, j \in N .
$$

Recall that the expected excess of a coalition $S$ with respect to a given allocation $x=(d, r)$ is $\mathbf{E}[e(S, x)]=\mathbf{E}[\mathscr{V}(S)]-d(S)$. If every player evaluates his negotiation power by means of total expected excess of coalitions containing him when distributing the worth $\mathbf{E}[\mathscr{V}(N)]$, then Equation 7.13 implies that all players have equal power with respect to the optimal solution. Thus the optimal solution not only implements the egalitarian idea towards the coalitions but also towards the individual players. Thus, the optimal solution to some extent strengthens the stability. Formally, we give the following definition.

Definition 7.4. For any $(N, \mathscr{V}) \in S G(N)$, the fairest solution $F(\mathscr{V})$ is defined as the optimal solution of Problem 7.3, i.e.,

$$
F_{i}(\mathscr{V})=\frac{1}{n} \mathbf{E}[\mathscr{V}(N)]+\frac{1}{n 2^{n-2}}\left[n e_{i}(\mathscr{V})-\sum_{j \in N} e_{j}(\mathscr{V})\right]+\frac{1}{n}\{\mathscr{V}(N)-\mathbf{E}[\mathscr{V}(N)]\}
$$

where $e_{i}(\mathscr{V})=\sum_{S \ni i} \mathbf{E}[\mathscr{V}(S)], \forall i \in N$.

Recall that the average excess $\bar{e}(\mathscr{V})$ of a game does not depend on the choice of allocation. This prompts us to think whether the optimal solution would change if we remove $\bar{e}(\mathscr{V})$ in the objective function of Problem 7.3, i.e.,

Problem 7.5.

$$
\begin{aligned}
& \min \sum_{S \subseteq N} \mathbf{E}\left[e^{2}(S, x)\right] \\
& \text { s.t. } \quad x \in D R(\mathscr{V}) .
\end{aligned}
$$

The essence of this problem is to select allocations which are closest to the original game from the expectation viewpoint. Interestingly, the optimal solution of Problem 7.5 is exactly the fairest solution of Problem 7.3. 
Theorem 7.4. For any $(N, \mathscr{V}) \in S G(N)$, the fairest solution $F(\mathscr{V})$ is the unique optimal solution of Problem 7.5.

Proof. Denote the objective function of Problem 7.5 as $P_{3}$, then we have

$$
\begin{aligned}
P_{3} & =\sum_{S \subseteq N} \mathbf{E}\left[(\mathscr{V}(S)-x(S))^{2}\right] \\
& =\sum_{S \subseteq N} \mathbf{E}\left[(\mathscr{V}(S)-d(S)-r(S) \mathscr{V}(N)+r(S) \mathbf{E}[\mathscr{V}(N)])^{2}\right] \\
& =\sum_{S \subseteq N} \mathbf{E}\left[(\mathscr{V}(S)-d(S))^{2}+r^{2}(S) \mathbf{D}[\mathscr{V}(N)]\right. \\
& -2 r(S)(\mathscr{V}(S)-d(S))(\mathscr{V}(N)-\mathbf{E}[\mathscr{V}(N)])] \\
& =\sum_{S \subseteq N}\left(\mathbf{E}\left[\mathscr{V}^{2}(S)\right]-2 d(S) \mathbf{E}[\mathscr{V}(S)]+d^{2}(S)+r^{2}(S) \mathbf{D}[\mathscr{V}(N)]\right)
\end{aligned}
$$

Due to the strict convexity of objective function $P_{3}$ and the feasible set, we conclude that there exists at most one optimal point. Imposing the Lagrangian multiplication factors $\lambda, \omega$, the Lagrangian function of Problem 7.5 is

$$
L(x, \lambda, \omega)=P_{3}+\lambda(d(N)-\mathbf{E}[\mathscr{V}(N)])+\omega(r(N)-1) .
$$

Taking the partial derivative with respect to $d_{i}$ and $r_{i}$ in Equation (7.15), the Lagrangian conditions are

$$
\begin{array}{r}
\left.L_{d_{i}}(x, \lambda, \omega)=\sum_{S \ni i}\{2 d(S)-2 \mathbf{E}[\mathscr{V}(S))]\right\}+\lambda=0, \forall i \in N, \\
L_{r_{i}}(x, \lambda, \omega)=\sum_{S \ni i} 2 r(S) \mathbf{D}[\mathscr{V}(N)]+\omega=0, \forall i \in N .
\end{array}
$$

Equations (7.16) and (7.17) imply the following relations among players.

$$
\begin{gathered}
\left.\left.\sum_{S \ni i}\{2 d(S)-2 \mathbf{E}[\mathscr{V}(S))]\right\}=\sum_{S \ni j}\{2 d(S)-2 \mathbf{E}[\mathscr{V}(S))]\right\}, \forall i, j \in N . \\
\sum_{S \ni i} 2 r(S) \mathbf{D}[\mathscr{V}(N)]=\sum_{S \ni j} 2 r(S) \mathbf{D}[\mathscr{V}(N)], \forall i, j \in N .
\end{gathered}
$$


Notice that the relations in Equations (7.18) and (7.19) are identical with those in Equations (7.11) and (7.12) respectively, therefore the optimal solution of Problem 7.5 is the same as Problem 7.3.

\subsection{Optimization models for stochastic cooperative games based on individual players}

So far, we have discussed different optimization models for stochastic cooperative games based on coalitions, and two solution concepts called the most stable solutions and the fairest solutions by solving the corresponding optimization problems. Generally, we measure the bargaining power of players through the worth of coalitions, but one thing that must be kept in mind is that the final decision-makers are the individual players. In this section, we focus on the optimization models and explore optimal solution concepts from the prospective of individual players. Interestingly, we can also implement the most stable solution and the fairest solution as the optimal points of these optimization problems involving the individual players.

Given any $(N, \mathscr{V}) \in S G(N)$ and $S \subseteq N$ with $i \in S$, the marginal contribution of player $i$ to coalition $S$, defined as $\mathscr{V}(S)-\mathscr{V}(S \backslash i)$, reflects the extra benefit when $i$ joins the formed coalition $S \backslash i$. For a potential payoff vector $x \in D R(\mathscr{V}), m_{i}(S, x):=\mathscr{V}(S)-\mathscr{V}(S \backslash i)-x_{i}$ can be regarded as measuring the dissatisfaction of player $i$ at $x$ with respective to coalition $S$. This motivates the following problem

Problem 7.6.

$$
\begin{aligned}
& \min \sum_{i \in N} \sum_{S \ni i} \mathbf{D}\left[m_{i}(S, x)\right] \\
& \text { s.t. } \quad x \in D R(\mathscr{V}) .
\end{aligned}
$$

Problem 7.6 is to select allocations in $D R(\mathscr{V})$ that minimize the total variances of excesses for individual players.

Theorem 7.5. For any $(N, \mathscr{V}) \in S G(N)$, the most stable solutions $M S(\mathscr{V})$ are the optimal solutions of Problem 7.6. 
Proof. Denote the objective function of Problem 7.6 as $P_{4}$. Then we have

$$
\begin{aligned}
P_{4} & =\sum_{i \in N} \sum_{S \ni i} \mathbf{D}\left[\mathscr{V}(S)-\mathscr{V}(S \backslash i)-d_{i}-r_{i} \mathscr{V}(N)+r_{i} \mathbf{E}[\mathscr{V}(N)]\right] \\
& =\sum_{i \in N} \sum_{S \ni i} \mathbf{D}\left[\mathscr{V}(S)-\mathscr{V}(S \backslash i)-r_{i} \mathscr{V}(N)\right] \\
& =\sum_{i \in N} \sum_{S \ni i}\left\{r_{i}^{2} \mathbf{D}[\mathscr{V}(N)]+\mathbf{D}[\mathscr{V}(S)-\mathscr{V}(S \backslash i)]-2 \operatorname{cov}(\mathscr{V}(S)-\mathscr{V}(S \backslash i), \mathscr{V}(N))\right\}
\end{aligned}
$$

Note that all the items except the first one are constants for any given $(N, \mathscr{V}) \in$ $S G(N)$, hence Problem 7.6 is equivalent to the following problem

\section{Problem 7.7.}

$$
\begin{aligned}
& \min \sum_{i \in N} \sum_{S \ni i} r_{i}^{2} \mathbf{D}[\mathscr{V}(N)] \\
& \text { s.t. } \quad \sum_{i \in N} r_{i}=1 \text { and } r_{i} \geq 0, \forall i \in N .
\end{aligned}
$$

Interestingly, the optimal solution coincides with that of Problem 7.1, i.e., the most stable solutions $M S(\mathscr{V})$ are the optimal solutions of Problem 7.6. One could easily verify the validity with the aid of Lagrange multiplier method by considering Problem 7.7, thus here we omit the details.

Furthermore, similar to the proposal of Problem 7.3, we consider the following problem

\section{Problem 7.8.}

$$
\begin{aligned}
& \min \sum_{i \in N} \sum_{S \ni i} \mathbf{E}\left[\left\{m_{i}(S, x)-\bar{m}(\mathscr{V}, x)\right\}^{2}\right] \\
& \text { s.t. } \quad x \in D R(\mathscr{V}),
\end{aligned}
$$

where $\bar{m}(\mathscr{V}, x)=\frac{1}{n 2^{n-1}} \sum_{i \in N} \sum_{S \ni i} m_{i}(S, x)$ is the average marginal excess at allocation $x$. In fact, for any $(N, \mathscr{V}) \in S G(N), \bar{m}(\mathscr{V}, x)$ does not depend on the choice of allocation. 
Proposition 7.2. For any $(N, \mathscr{V}) \in S G(N)$, the average marginal excess is the same for all allocations in $D R(\mathscr{V})$.

Proof. Given any $x \in D R(\mathscr{V})$, then

$$
\begin{aligned}
& \bar{m}(\mathscr{V}, x)=\frac{1}{n 2^{n-1}} \sum_{i \in N} \sum_{S \ni i}\left\{\mathscr{V}(S)-\mathscr{V}(S \backslash i)-x_{i}\right\} \\
= & \frac{1}{n 2^{n-1}} \sum_{i \in N} \sum_{S \ni i}\{\mathscr{V}(S)-\mathscr{V}(S \backslash i)\}-\frac{1}{n 2^{n-1}} \sum_{i \in N} \sum_{S \ni i} x_{i} \\
= & \frac{1}{n 2^{n-1}} \sum_{S \subseteq N} s \mathscr{V}(S)-\frac{1}{n 2^{n-1}} \sum_{S \subseteq N}(n-s) \mathscr{V}(S)-\frac{1}{n 2^{n-1}} 2^{n-1} \mathscr{V}(N) \\
= & \frac{1}{n 2^{n-1}} \sum_{S \subseteq N}(2 s-n) \mathscr{V}(S)-\frac{1}{n} \mathscr{V}(N) .
\end{aligned}
$$

Based on the above result, we write $\bar{m}(\mathscr{V})$ to represent the average marginal excess for game $\mathscr{V}$. The next proposition reveals that the optimal solution of Problem 7.8 would not change if we abandon the "constant" item $\bar{m}(\mathscr{V})$ in the objective function. Interestingly, the optimal solution of this problem coincides with those of Problem 7.3 and Problem 7.5.

Theorem 7.6. For any $(N, \mathscr{V}) \in S G(N)$, the fairest solution $F(\mathscr{V})$ is the unique optimal solution of Problem 7.8.

Theorem 7.6 reveals the fact that all roads lead to Rome. When considering the least square criterion of complaint, whether from the perspective of coalitions or individuals, it eventually reaches the same destination, that is, the fairest solution. To verify the validity of Theorem 7.6, we first present the following lemma.

Lemma 7.7. Problem 7.8 is equivalent to the following problem.

\section{Problem 7.9.}

$$
\begin{aligned}
& \min \sum_{i \in N} \sum_{S \ni i} \mathbf{E}\left[\left\{m_{i}(S, x)\right\}^{2}\right] \\
& \text { s.t. } \quad x \in D R(\mathscr{V}) .
\end{aligned}
$$


Proof. Denote the objective function of Problem 7.8 as $P_{5}$, then we have

$$
\begin{aligned}
P_{5} & =\sum_{i \in N} \sum_{S \ni i} \mathbf{E}\left[\left\{m_{i}(S, x)-\bar{m}(\mathscr{V})\right\}^{2}\right] \\
& =\sum_{i \in N} \sum_{S \ni i} \mathbf{E}\left[m_{i}^{2}(S, x)+\bar{m}^{2}(\mathscr{V})-2 m_{i}(S, x) \bar{m}(\mathscr{V})\right] \\
& =\sum_{i \in N} \sum_{S \ni i} \mathbf{E}\left[m_{i}^{2}(S, x)\right]+\underbrace{\sum_{i \in N} \sum_{S \ni i} \mathbf{E}\left[\bar{m}^{2}(\mathscr{V})\right]}_{P_{5}^{1}}-\underbrace{2 \sum_{i \in N} \sum_{S \ni i} \mathbf{E}\left[m_{i}(S, x) \bar{m}(\mathscr{V})\right]}_{P_{5}^{2}}
\end{aligned}
$$

As declared in Proposition 7.2, $\bar{m}(\mathscr{V})$ only depends on the game $\mathscr{V}$, eliminating the item $P_{5}^{1}$ has no effect no the optimal solution of Problem 7.8. Now we focus on the item $P_{5}^{2}$, which can be rewritten as:

$$
\begin{aligned}
P_{5}^{2} & =2 \sum_{i \in N} \sum_{S \ni i} \mathbf{E}\left[\left\{\mathscr{V}(S)-\mathscr{V}(S \backslash i)-x_{i}\right\} \bar{m}(\mathscr{V})\right] \\
& =2 \sum_{i \in N} \sum_{S \ni i} \mathbf{E}[\{\mathscr{V}(S)-\mathscr{V}(S \backslash i)\} \bar{m}(\mathscr{V})] \\
& -2 \sum_{i \in N} \sum_{S \ni i} d_{i} \mathbf{E}[\bar{m}(\mathscr{V})]-2 \sum_{i \in N} \sum_{S \ni i} r_{i} \mathbf{E}[\{\mathscr{V}(N)-\mathbf{E}[\mathscr{V}(N)]\} \bar{m}(\mathscr{V})] \\
& =2 \sum_{i \in N} \sum_{S \ni i} \mathbf{E}[\{\mathscr{V}(S)-\mathscr{V}(S \backslash i)\} \bar{m}(\mathscr{V})]-2^{n} \mathbf{E}[\{\mathscr{V}(N)] \mathrm{E}[\bar{m}(\mathscr{V})] \\
& -2{ }^{n} \mathbf{E}[\{\mathscr{V}(N)-\mathbf{E}[\mathscr{V}(N)]\} \bar{m}(\mathscr{V})]
\end{aligned}
$$

The last equation is derived from the fact that $x=(d, r) \in D R(\mathscr{V})$ satisfying $d(N)=\mathbf{E}[\{\mathscr{V}(N)]$ and $r(N)=1$. It immediately follows that eliminating the constant items $P_{5}^{1}$ and $P_{5}^{2}$ from the objective function of Problem 7.8 exerts no influence on the optimal solution, which completes the proof.

Now we restrict our attention on Problem 7.9 of which the optimal solution is exhibited.

Lemma 7.8. For any $(N, \mathscr{V}) \in S G(N)$, the fairest solution $F(\mathscr{V})$ is the unique optimal solution of Problem 7.9. 
Proof. Denote the objective function of Problem 7.9 as $P_{6}$. Then we have

$$
\begin{aligned}
P_{6} & =\sum_{i \in N} \sum_{S \ni i} \mathbf{E}\left[\{\mathscr{V}(S)-\mathscr{V}(S \backslash i)-x(S)+x(S \backslash i)\}^{2}\right] \\
& =\sum_{i \in N} \sum_{S \ni i} \mathbf{E}\left[\{e(S, x)-e(S \backslash i, x)\}^{2}\right] \\
& =\sum_{i \in N} \sum_{S \ni i} \mathbf{E}\left[e^{2}(S, x)+e^{2}(S \backslash i, x)-2 e(S, x) e(S \backslash i, x)\right] \\
& =\sum_{S \subseteq N} s \mathbf{E}\left[e^{2}(S, x)\right]+\sum_{S \subseteq N}(n-s) \mathbf{E}\left[e^{2}(S, x)\right] \\
& -2 \sum_{i \in N} \sum_{S \ni i} \mathbf{E}[e(S, x) e(S \backslash i, x)] \\
& =n \underbrace{2}_{\sum_{S \subseteq N} \mathbf{E}\left[e^{2}(S, x)\right]} \underbrace{\sum_{i \in N} \sum_{S \ni i} \mathbf{E}[e(S, x) e(S \backslash i, x)]}_{P_{61}} \\
& =n P_{61}-2 P_{62},
\end{aligned}
$$

where the second formulation $P_{62}$ satisfies

$$
\begin{aligned}
P_{62} & =\sum_{i \in N} \sum_{S \ni i} \mathbf{E}[\{(\mathscr{V}(S)-x(S))(\mathscr{V}(S \backslash i)-x(S \backslash i))\}] \\
& =\sum_{i \in N} \sum_{S \ni i} \mathbf{E}[\mathscr{V}(S) \mathscr{V}(S \backslash i)]-\sum_{i \in N} \sum_{S \ni i} \mathbf{E}[\mathscr{V}(S) x(S \backslash i)] \\
& -\sum_{i \in N} \sum_{S \ni i} \mathbf{E}[\mathscr{V}(S \backslash i) x(S)]+\sum_{i \in N} \sum_{S \ni i} \mathbf{E}[x(S) x(S \backslash i)] \\
& =\sum_{i \in N} \sum_{S \ni i} \mathbf{E}[\mathscr{V}(S) \mathscr{V}(S \backslash i)]+\sum_{i \in N} \sum_{S \ni i} \mathbf{E}[x(S) x(S \backslash i)] \\
& -\sum_{i \in N} \sum_{S \ni i} \mathbf{E}[\mathscr{V}(S) x(S)]+\sum_{i \in N} \sum_{S \ni i} \mathbf{E}\left[\mathscr{V}(S) x_{i}\right] \\
& -\sum_{i \in N} \sum_{S \ni i} \mathbf{E}[\mathscr{V}(S \backslash i) x(S \backslash i)]-\sum_{i \in N} \sum_{S \ni i} \mathbf{E}\left[\mathscr{V}(S \backslash i) x_{i}\right] \\
& =\sum_{i \in N} \sum_{S \ni i} \mathbf{E}[\mathscr{V}(S) \mathscr{V}(S \backslash i)]+\sum_{i \in N} \sum_{S \ni i} \mathbf{E}[x(S) x(S \backslash i)] \\
& -\sum_{S \subseteq N} s \mathbf{E}[\mathscr{V}(S) x(S)]+\sum_{i \in N} \sum_{S \ni i} \mathbf{E}\left[\mathscr{V}(S) x_{i}\right] \\
& -\sum_{S \subseteq N}(n-s) \mathbf{E}[\mathscr{V}(S) x(S)]-\sum_{S \subseteq N} \mathbf{E}[\mathscr{V}(S)(\mathscr{V}(N)-x(S))]
\end{aligned}
$$




$$
\begin{aligned}
& =\sum_{i \in N} \sum_{S \ni i} \mathbf{E}[\mathscr{V}(S) \mathscr{V}(S \backslash i)]+\underbrace{\sum_{i \in N} \sum_{S \ni i} \mathbf{E}[x(S) x(S \backslash i)]}_{P_{62}^{1}}+\underbrace{\sum_{i \in N} \sum_{S \ni i} \mathbf{E}\left[\mathscr{V}(S) x_{i}\right]}_{P_{62}^{2}} \\
& -\sum_{S \subseteq N}(n-1) \mathbf{E}[\mathscr{V}(S) x(S)]-\sum_{S \subseteq N} \mathbf{E}[\mathscr{V}(S) \mathscr{V}(N)] .
\end{aligned}
$$

Next, we analyze the items $P_{62}^{1}$ and $P_{62}^{2}$ respectively. By taking into account the fact that $x_{i}=d_{i}+r_{i}(\mathscr{V}(N)-\mathbf{E}[\mathscr{V}(N)])$, we have

$$
\begin{aligned}
P_{62}^{1} & =\sum_{i \in N} \sum_{S \ni i} \mathbf{E}[\{d(S)+r(S)(\mathscr{V}(N)-\mathbf{E}[\mathscr{V}(N)])\} \\
& =\sum_{i \in N} \sum_{S \ni i} \mathbf{E}\left[d(S) d(S \backslash i)+r(S) r(S \backslash i)(\mathscr{V}(N)-\mathbf{E}[\mathscr{V}(N)])^{2}\right. \\
& +\{(d(S) r(S \backslash i)+r(S) d(S \backslash i))(\mathscr{V}(N)-\mathbf{E}[\mathscr{V}(N)])\}] \\
& \left.=\sum_{i \in N} \sum_{S \ni i}\{d(S) d(S \backslash i)+r(S) r(S \backslash i)) \mathbf{D}[\mathscr{V}(N)]\right\} \\
& =\frac{1}{2} \sum_{i \in N} \sum_{S \ni i} d(S)\left(d(S)-d_{i}\right)+\frac{1}{2} \sum_{i \in N} \sum_{S \ni i}\left(d(S \backslash i)+d_{i}\right) d(S \backslash i) \\
& +\frac{1}{2} \sum_{i \in N} \sum_{S \ni i} r(S)\left(r(S)-r_{i}\right) \mathbf{D}[\mathscr{V}(N)] \\
& +\frac{1}{2} \sum_{i \in N} \sum_{S \ni i}\left(r(S \backslash i)+r_{i}\right) r(S \backslash i) \mathbf{D}[\mathscr{V}(N)] \\
= & \frac{1}{2} \sum_{S \subseteq N} s\left(d^{2}(S)+r^{2}(S) \mathbf{D}[\mathscr{V}(N)]\right) \\
+ & \frac{1}{2} \sum_{S \subseteq N}(n-s)\left(d^{2}(S)+r^{2}(S) \mathbf{D}[\mathscr{V}(N)]\right) \\
& -\frac{1}{2} \sum_{S \subseteq N}\left(d^{2}(S)+r^{2}(S) \mathbf{D}[\mathscr{V}(N)]\right) \\
+ & \frac{1}{2} \sum_{S \subseteq N}(d(N \backslash S) d(S)+r(N \backslash S) r(S) \mathbf{D}[\mathscr{V}(N)]) \\
= & \frac{1}{2} \sum_{S \subseteq N}(n-1)\left(d^{2}(S)+r^{2}(S) \mathbf{D}[\mathscr{V}(N)]\right)
\end{aligned}
$$




$$
\begin{aligned}
& +\frac{1}{2} \sum_{S \subseteq N}\{(\mathbf{E}[\mathscr{V}(N)]-d(S)) d(S)+(1-r(S)) r(S) \mathbf{D}[\mathscr{V}(N)]\} \\
& =\frac{1}{2} \sum_{S \subseteq N}(n-2)\left(d^{2}(S)+r^{2}(S) \mathbf{D}[\mathscr{V}(N)]\right) \\
& +\frac{1}{2} \sum_{S \subseteq N} d(S) \mathbf{E}[\mathscr{V}(N)]+\frac{1}{2} \sum_{S \subseteq N} r(S) \mathbf{D}[\mathscr{V}(N)] \\
& =\frac{1}{2} \sum_{S \subseteq N}(n-2) \mathbf{E}\left[x^{2}(S)\right]+2^{n-2} \mathbf{E}^{2}[\mathscr{V}(N)]+2^{n-2} \mathbf{D}[\mathscr{V}(N)]
\end{aligned}
$$

Moreover, the formulation $P_{62}^{2}$ can be rewritten as follows.

$$
\begin{aligned}
P_{62}^{2} & =\sum_{i \in N} \sum_{S \ni i, S \neq N} \mathbf{E}\left[\mathscr{V}(S) x_{i}\right]+\sum_{i \in N} \mathbf{E}\left[\mathscr{V}(N) x_{i}\right] \\
& =\sum_{S \neq N} \mathbf{E}[x(S)] \mathbf{E}[\mathscr{V}(S)]+\sum_{i \in N} \mathbf{E}\left[\left\{d_{i}+r_{i} \mathscr{V}(N)-r_{i} \mathbf{E}[\mathscr{V}(N)]\right\} \mathscr{V}(N)\right] \\
& =\sum_{S \neq N} \mathbf{E}[x(S)] \mathbf{E}[\mathscr{V}(S)]+\sum_{i \in N} d_{i} \mathbf{E}[\mathscr{V}(N)] \\
& +\sum_{i \in N} r_{i}\left\{\mathbf{E}\left[\mathscr{V}^{2}(N)\right]-\mathbf{E}^{2}[\mathscr{V}(N)]\right\} .
\end{aligned}
$$

Recall that $\sum_{i \in N} d_{i}=\mathbf{E}[\mathscr{V}(N)]$ and $\sum_{i \in N} r_{i}=1$. Thus we have

$$
P_{62}^{2}=\sum_{S \neq N} \mathrm{E}[x(S)] \mathrm{E}[\mathscr{V}(S)]+\mathbf{E}\left[\mathscr{V}^{2}(N)\right]=\sum_{S \subseteq N} \mathbf{E}[x(S) \mathscr{V}(S)] .
$$

Based on the above analysis, it holds that

$$
\begin{aligned}
P_{6} & =n P_{61}-2 P_{62} \\
& =n \sum_{S \subseteq N} \mathbf{E}\left[e^{2}(S, x)\right]-2 \sum_{i \in N} \sum_{S \ni i} \mathbf{E}[\mathscr{V}(S) \mathscr{V}(S \backslash i)]-2 P_{62}^{1}-2 P_{62}^{2} \\
& +2 \sum_{S \subseteq N}(n-1) \mathbf{E}[\mathscr{V}(S) x(S)]+2 \sum_{S \subseteq N} \mathbf{E}[\mathscr{V}(S) \mathscr{V}(N)] \\
& =n \sum_{S \subseteq N} \mathbf{E}\left[e^{2}(S, x)\right]-2 \sum_{i \in N} \sum_{S \ni i} \mathbf{E}[\mathscr{V}(S) \mathscr{V}(S \backslash i)] \\
& -\sum_{S \subseteq N}(n-2) \mathbf{E}\left[x^{2}(S)\right]-2^{n-1} \mathbf{E}^{2}[\mathscr{V}(N)]-2^{n-1} \mathbf{D}[\mathscr{V}(N)]
\end{aligned}
$$




$$
\begin{aligned}
& +2(n-2) \sum_{S \subseteq N} \mathbf{E}[\mathscr{V}(S) x(S)]+2 \sum_{S \subseteq N} \mathbf{E}[\mathscr{V}(S) \mathscr{V}(N)] \\
& =n \sum_{S \subseteq N} \mathbf{E}\left[e^{2}(S, x)\right]-2 \sum_{i \in N} \sum_{S \ni i} \mathbf{E}[\mathscr{V}(S) \mathscr{V}(S \backslash i)] \\
& -(n-2) \sum_{S \subseteq N} \mathbf{E}\left[e^{2}(S, x)\right]+(n-2) \sum_{S \subseteq N} \mathbf{E}\left[\mathscr{V}^{2}(S)\right] \\
& -2^{n-1} \mathbf{E}^{2}[\mathscr{V}(N)]-2^{n-1} \mathbf{D}[\mathscr{V}(N)] \\
& =2 \sum_{S \subseteq N} \mathbf{E}\left[e^{2}(S, x)\right]-2 \sum_{i \in N} \sum_{S \ni i} \mathbf{E}[\mathscr{V}(S) \mathscr{V}(S \backslash i)] \\
& -2^{n-1} \mathbf{E}^{2}[\mathscr{V}(N)]-2^{n-1} \mathbf{D}[\mathscr{V}(N)] \\
& \triangleq 2 \sum_{S \subseteq N} \mathrm{E}\left[e^{2}(S, x)\right]+k(\mathscr{V}) \text {, }
\end{aligned}
$$

where $k(\mathscr{V})=-2 \sum_{i \in N} \sum_{S \ni i} \mathbf{E}[\mathscr{V}(S) \mathscr{V}(S \backslash i)]-2^{n-1} \mathbf{E}^{2}[\mathscr{V}(N)]-2^{n-1} \mathbf{D}[\mathscr{V}(N)]$. Note that for any given $(N, \mathscr{V}) \in S G(N), k(v)$ is a constant, which means that there exists a positive linear relation between the objective function $P_{6}$ of Problem 7.9 and that of Problem 7.5. Therefore the fairest solution is the optimal solution of Problem 7.9.

In essence, there exists a linear relation with positive coefficient between the objective functions of Problem 7.9 and Problem 7.5. Therefore the optimal solution of Problem 7.9 is the same as Problem 7.5. Together with Theorem 7.4 and Lemma 7.7, we conclude that the fairest solution is the unique optimal solution of Problem 7.8 and thus Theorem 7.6 is demonstrated. 



\section{Summary}

This thesis mainly focuses on solution concepts for cooperative games. We try to investigate the solution concepts concerning the complaints of players. Various principles are taken into account in order to define the complaints for either individual players or coalitions. Then based on different optimization criteria, values are defined as the solutions to corresponding optimization models. Alternatively, we deal with the complaints in the view of compromise motivated by the definition of the $\tau$ value [81]. In such a way, we define a more general compromise value, which has a very close relation to many well-known compromise values.

In Chapter 2, motivated by the work of Malawski [47] on procedural value, we study the formation of the grand coalition and define a new kind of complaint for individual players, which is in some sense a compromise among all players when considering all possible permutations. Followed by the definition of the (pre-)nucleolus [71] and the family of least square values [69], we then reveal that the optimal solutions for both models coincide with the ENSC value either based on the lexicographic criterion or the least square criterion. We also characterize the ENSC value as the unique satisfier of a particular collection of axioms.

Furthermore, in Chapter 3, we propose the so called $\alpha$-ENSC value by considering the egoism of players. First of all, the coefficient $\alpha$ is introduced to measure the degree of egoism of players. Then similar to the procedural method in Chapter 2, we implement the $\alpha$-ENSC value by means of optimization and also the satisfier of a set of properties. Notice that, we can also implement the corresponding ( $\alpha$-)CIS value ( [24], [97]) due to the fact that 
the ENSC value and the CIS value are dual to each other. Following the similar idea, in Chapter 6, we propose another two kinds of complaints for coalitions and define the optimal compromise values based on the lexicographic criterion. It turns out that the optimal compromise values coincides with the ENSC value and the CIS value under corresponding complaint. The determination of other solution concepts from the perspective of optimization and procedure method is expected, such as the solidarity value [57] and the Banzhaf value [6]. The supreme step to achieve this is to figure out the corresponding complaint criterion.

In Chapter 4, we show an application of the method studied in Chapters 2 and 3. We introduce and axiomatize a class of cost sharing methods for polluted river sharing systems that consists of the convex combinations of the known Local Responsibility Sharing (LR) method and the Upstream Equal Sharing (UES) method introduced by Ni and Wang [55]. Notice that the structure of the river in our model is simply a straight line. For further research, we can consider some more complicated structure, which may be more related to the real natural structure, such as the tree-network structure.

Chapter 5 deals with the solution concepts based on the compromise between the ideal and minimal payoffs for players, which is inspired by the definition of the $\tau$ value [81] but in a more general way. We reveal the relations between the general compromise value with several well known solution concepts. For instance, we give the necessary and sufficient condition ensuring that the new value belongs to the core. We also provide sufficient conditions when the general compromise value coincides with the $\tau$ value and the $\chi$ value [8] respectively. For further research, one could explore the choice of the potential payoff sets, which leads to other solution concepts, such as the Shapley value and the solidarity value. As to the axiomatization of the general compromise value, one could also apply the method that characterizes the $\tau$ value on a set of games with a variable number of players [25].

Chapter 7 investigates the solution concepts for cooperative games with stochastic payoffs. We focus on a subset of all allocations and introduce the stochastic complaint for players. Under the least square criterion, the most stable solutions and the fairest solutions are proposed. Moreover, the optimal 
solution stays the same whether the optimization model depends on the coalitions or individual players. For further research, there is still a long way to go in this area. The first concern is how to axiomatize these solutions, where the main difficulty is the stochastic property of the payoffs. Another question is that whether the results of this chapter are valid for a more general class of allocations. We could also investigate the relationship between the proposed solution concepts and existing solution concepts, for instance, various core concepts for stochastic cooperative games. 



\section{Bibliography}

[1] Alcalde-Unzu J., Góez-Rúa M. and Molis E.: Sharing the cost of cleaning a river: The Upstream Responsibility rule. Games Econ Behav 90, 134150 (2015).

[2] Ambec S. and Ehlers L.: Sharing a river among satiable agents. Games Econ Behav 64, 35-50 (2008).

[3] Ambec S. and Sprumont Y.: Sharing a river. J Econ Theory 107, 453-462 (2002).

[4] Aumann R.J. and Maschler M.: Game theoretic analysis of a bankruptcy problem from the Talmud. Journal of Economic Theory 36,195-213 (1985).

[5] Aumann R.J.B. and Peleg B.: Von Neumann-Morgenstern solutions to cooperative games without side payments. Bulletin of the American Mathematical Society 66,173-179 (1960).

[6] Banzhaf J.F: Weighted voting doesn't work: A mathematical analysis. Rutgers Law Review 19, 317-343 (1965).

[7] Barrett S.: Conflict and cooperation in managing international water resources. Working paper 1303. World Bank, Washington (1994).

[8] Bergantiños G. and Massó J.: Notes on a new compromise value: the $\chi$-value. Optimization 38, 277-286 (1996). 
[9] Bergantiños G. and Massó J.: The Chi-compromise value for nontransferable utility games. Math Meth Oper Res 56, 269-286 (2002).

[10] Borm P., Keiding H., Mclean R.P., Oortwijn S. and Tijs S.H.: The compromise value for NTU-games. Int J Game Theory 21, 175-189 (1992).

[11] Branzei R., Dimitrov D. and Tijs S.H.: Models in cooperative game theory. Springer, Berlin OR Spectrum 7, 19-26 (2008).

[12] Casajus A. and Huettner F: Null, nullifying, or dummifying players: The difference between the Shapley value, the equal division value, and the equal surplus division value. Econom Lett 122, 167-169 (2014).

[13] Charnes A., and Granot D.: Prior solutions: Extensions of convex nucleolus solutions to chance-constrained games. Proceeding of the Computer Science and Statistics Seventh Stmposium at Iowa State University, 323-332 (1973).

[14] Charnes A., and Granot D.: Coalitional and chance-constrained solutions to N-person games I: The prior satisfying nucleolus. SIAM J Appl Math 31, 358-367 (1976).

[15] Charnes A., and Granot D.: Coalitional and chance-constrained solutions to N-person games II: Two-stage solutions. Oper Res 25, 10131019 (1977).

[16] Chetty V.K., Dasgupta D. and Raghavan T.E.S.: Power and distribution of profits. Discussion Paper no. 139, Indian Statistical Institute, Delhi Centre, New Delhi (1976).

[17] Crawford V.P: A procedure for generating Pareto-efficient egalitarianequivalent allocations. Econometrica 47, 49-60 (1979).

[18] Curiel I., Pederzoli G., and Tijs S.H.: Sequencing games. Eur J Oper Res 40, 344-351 (1989).

[19] Davis M. and Maschler M.: The kernel of a cooperative game. Naval Research Logistics Quarterly 12, 223-259 (1965). 
[20] Dong B., Ni D. and Wang, Y.: Sharing a polluted river network. Environ. Resource Econ. 53, 367-387 (2012).

[21] Driessen T.S.H. and Tijs S.H.: The $\tau$-value, the nucleolus and the core for a subclass of games. Tilburg University, School of Economics and Management (1983).

[22] Driessen T.S.H.: Properties of 1-convex $n$ person games. OR Spectrum 7, 19-26 (1985).

[23] Driessen T.S.H.: Cooperative Games, Solutions and Applications. Kluwer Academic Publishers, Dordrecht, The Netherlands (1988).

[24] Driessen T.S.H. and Funaki Y.: Coincidence of and collinearity between game theoretic solutions, OR Spectrum 13, 15-30 (1991).

[25] Driessen T.S.H. : On the reduced game property for and the axiomatization of the $\tau$-value of TU-games. Top 4,165-185 (1996).

[26] Driessen T.S.H., Khmelnitskaya A.B. and Jordi S.: 1-concave basis for TU games and the library game. TOP 20, 578-591 (2012).

[27] Evans R.A. Coalitions bargaining with competition to make offers, Games Econ Behav 19, 211-220 (1997).

[28] Fernandez F.R., Puerto J. and Zafra M.J.: Cores of stochastic cooperative games with stochastic orders. International Game Theory Review. 4, 265-280 (2002).

[29] Gillies D.B.: Some theorems on $n$ person games. Ph.D. Thesis. Princeton Uni- versity Press, Princeton, New Jersey, (1953).

[30] Godana B.: Africas Shared Water Resources. France Printer, London (1985).

[31] Granot D.: Cooperative games in stochastic function form. Manage Sci 23, 621-630 (1977). 
[32] Grotte J.: Computation of and Observations on the Nucleolus, the Normalized Nucleolus and the Central Games, Cornell University Ithaca (1970).

[33] Habis H. and Herings P.J.J.: Core concepts for incomplete market economics. J Math Econ 47, 595-609 (2011).

[34] Habis H. and Herings P.J.J.: Transferable utility games with uncertainty. J Econ Theory 146, 2126-2139 (2011).

[35] Habis H. and Herings P.J.J.: Stochastic bankruptcy games. Int J Game Theory 42, 973-988 (2013).

[36] Hamiache G.: Associated consistency and Shapley value. Int J Game Theory 30, 279-289 (2001).

[37] Harsanyi J.C.: A bargaining model for cooperative n-person games, in:A.W. Tucker,R.D.Luce(Eds.), Contributions to the theory of games IV, Priceton University Press, Priceton, 325-355 (1959).

[38] Heumen H. van: The $\mu$-value: a solution concept for cooperative games. Master Thesis, University of Nijmegen, Nijmegen, the Netherlands (1984).

[39] Hou D., Sun P., Xu G. and Driessen T.S.H.: Compromise for the complaint: an optimization approach to the ENSC value and the CIS value. J Oper Res Soc 69(4), 571-579 (2017).

[40] Joosten R., Peters H. and Thuijsman F.: Socially acceptable values for transferable utility games, Report M94-03, Department of Mathematics, University of Maastricht (1994).

[41] Joosten R.: "Dynamics, Equilibria, and Values", Ph.D. Thesis, Maastricht University (1996).

[42] Ju Y. and Wettstein D.: Implementing cooperative solution concepts: a generalized bidding approach. Econ Theory 39, 307-330 (2009). 
[43] Kalai E. and Smorodinsky M.: Other solutions to Nash's bargaining problem. Econometrica 43, 513-528 (1975).

[44] Khmelnitskaya A.B.: Values for rooted-tree and sink-tree digraphs games and sharing a river. Theory Dec 69,657-669 (2010).

[45] Kilgour M. and Dinar A.: Are stable agreements for sharing international river waters now possible? Working paper 1474. World Bank, Washington (1996).

[46] Littlechild S.C. and Owen, G.: A simple expression for the Shapley value in a special case. Management Sci 20, 370-372 (1973).

[47] Malawski M.: "Procedural" values for cooperative games. Int J Game Theory. 42, 305-324 (2013).

[48] Maschler M., Peleg B. and Shapley L.S.: The Kernel and bargaining set for convex games. Int J Game Theory 1, 73-93 (1972).

[49] Maschler M.: An advantage of the bargaining set over the core. J Econom Theory 13, 184-192 (1976).

[50] Maschler M. and Peleg B.: A characterization existence proof and dimension bounds for the kernel of a game. Pacific J Math 18, 289-323 (1966).

[51] Maschler M., Peleg B., and Shapley L.S.: Geometric properties of the Kernel, Nucleolus, and related solution concepts. Math Oper Res 4, 303338 (1979).

[52] Milnor J.W.: Reasonable outcomes for n-person games. Research Memodrandum RM-916, the Rand Coporation, Santa Monica (1952).

[53] Moulin H.: The separability axiom and equal-sharing methods, J Econ Theory 36, 120-148 (1985).

[54] Nash J.F: The bargaining problem. Econometrica 18, 155-162 (1950).

[55] Ni D. and Wang Y.: Sharing a polluted river. Games Econ Behav 60, 176-186 (2007). 
[56] Neumann J. von and Morgenstern, O.: Theory of games and economic behavior. Princeton university Press (1944).

[57] Nowak A., Radzik T.: A solidarity value for n-person TU games. Int $\mathrm{J}$ Game Theory 23, 43-48 (1994).

[58] Oishi T., Nakayama M., Hokari T. and Funaki Y.: Duality and anti-duality in TU games applied to solutions, axioms, and axiomatizations. J Math Econ 63, 44-53 (2016).

[59] O'Neill B.: A problem of rights arbitration from the Talmud. Math Social Sci 22, 741-750 (1982).

[60] özen U., Fransoo J., Norde H. and Slikker M.: Cooperation between multiple newsvendors with warehouses. Manufacturing and Service Operations Management. 10, 311-324 (2008).

[61] Pérez-Castrillo D. and Wettstein, D.: Bidding for the surplus: a noncooperative approach to the Shapley value. J Econ Theory 100, 274-294 (2001).

[62] Pérez-Castrillo D. and Wettstein, D.: Choosing wisely: a multi-bidding approach. Am Econ Rev 92, 1577- 1587 (2002).

[63] Predtetchinski A.: The strong sequential core for stationary cooperative games. Games Econ Behav 61, 50-66 (2007).

[64] Raiffa H.: Arbitration schemes for generalized two-person games. Ann of Math Stud 28, 361-387 (1953).

[65] Ransmeier J.S.: The Tennessee Valley Authority: a case study in the economics of multiple purpose stream planning. The Vanderbilt University Press (1942).

[66] Roger B.M.: Game Theory: Analysis of Conflict. Harvard University Press, Cambridge (1991).

[67] Rosenmüller J.: Kooperative Spiele and Märkte. Springer-Verlag, Berlin (1971). 
[68] Ruiz L.M., Valenciano F., Zarzuelo J.M.: The least square prenucleolus and the least square nucleolus. Int J Game Theory 25, 113-134 (1996).

[69] Ruiz L.M., Valenciano F., Zarzuelo J.M.: The family of least square values for transferable utility games. Games Econ Behav 24, 109-130 (1998a).

[70] Ruiz L.M., Valenciano F., Zarzuelo J.M.: Some new results on least square values for TU games. TOP 6, 139-158 (1998b).

[71] Schmeidler D.: The nucleolus of a characteristic function game. SIAM J Appl Math 17, 517-520 (1969).

[72] Shapley L.S.: A value for n-person Games. Annals of Mathematical Studies 28, 307-317 (1953).

[73] Shapley L.S.: Cores of convex games. Int J Game Theory 1, 11-26 (1971).

[74] Shapley L.S. and Shubik M.: Ownership and the production function. Quart J Econom 81, 88-111 (1967).

[75] Sobolev A.I.: The characterization of optimality principles in cooperative games by functional equations (in Russian). Vorobjev NN(Ed) Mathematical Methods in the Social Sciences 6, Academy of Sciences of the Lithuanian SSR, Vilnius 94-151 (1975).

[76] Suijs J. and Borm P.: Cooperative Games with Stochastic Payoffs: Deterministic Equivalents. (FEW Research Memorandum; Vol. 713). Tilburg: Operations research (1996).

[77] Suijs J., Borm P., Waegenaere D.A. and Tijs, S.H.: Cooperative games with stochastic payoffs. Eur J Oper Res 113, 193-205 (1999).

[78] Suijs J.: Price uncertainty in linear production situations. Cooperative Decision-Making Under Risk 63-87, Kluwer (2000).

[79] Sun P., Hou D., Sun H. and Driessen T.S.H.: Optimization implementation and characterization of the equal allocation of non-separable costs value. J Optim THeory 173, 336-352 (2017). 
[80] Sun P., Hou D., Sun H. and Zhang H.: Process and optimization implementation of the $\alpha$-ENSC value. Math Meth Oper Res 86, 293-308 (2017).

[81] Tijs S.H.: Bounds for the core of a game and the $\tau$-value. Game Theory and Mathematical Economics. North-Holland Publishing Company, Amsterdam, 123-132 (1981).

[82] Tijs S.H.: An axiomatization of the $\tau$-value. Math Social Sci 13: 177181 (1987).

[83] Tijs S.H. and Driessen T.S.H.: The $\tau$-value as a feasible compromise between Utopia and disagreement. Axiomatics and Pragmatics of Conflict Analysis. Gower Publishing Company, Aldershot, England, 142-156 (1987).

[84] Tijs S.H. and Otten G.J.: Compromise values in cooperative game theory. TOP 1,1-36 (1993).

[85] Timmer J.: The compromise value for cooperative games with random payoffs. Math Meth Oper Res 64, 95-106 (2006).

[86] Toriello A. and Uhan N.A.: 2017. Dynamic linear programming games with risk-averse players. Math Program Ser A 163, 25-56 (2017).

[87] Uhan N.A.: Stochastic linear programming games with concave preferences. Eur J Oper Res 243, 637-646 (2015).

[88] van den Brink R.: Null or nullifying players: the difference between the Shapley value and the equal division solutions. J Econ Theory 136, 767-775 (2007).

[89] van den Brink R., Funaki Y.: Axiomatizations of a class of equal surplus sharing solutions for cooperative games with transferable utility. Theory Decis 67, 303-340 (2009).

[90] van den Brink R., Funaki Y.: Reconciling marginalism with egalitarianism: consistency, monotonicity, and implementation of egalitarian Shapley values. Soc Choice Welf 40, 693-714 (2013). 
[91] van den Brink R. and Gilles R.P.: Axiomatizations of the conjunctive permission value for games with permission structures. Games Econ Behav 12, 113-126 (1996).

[92] van den Brink R. and van der Laan G.: Comment on "Sharing a polluted river". Mimeo (2008).

[93] van den Brink R., Chun Y., Funaki Y. and Park B.: Consistency, population solidarity, and egalitarian solutions for TU-Games. Theory Decis 81, 427-447 (2016).

[94] van den Brink R., van der Laan G. and Moes N.: Fair agreements for sharing international rivers with multiple springs and externalities. $\mathrm{J}$ Environ Econ Manage 3, 388-403 (2012).

[95] van den Brink R., He S. and Huang J.: Polluted river problems and games with a permission structure. Games Econ Behav 108, 182-205 (2018).

[96] Weber R.J.: Probabilistic values for games. In: Roth AE(ed) The Shapley value: essays in hornor of Lloyd Shapley, Cambridge University Press, Cambridge (1988).

[97] Xu G., Dai H. and Shi H.: Axiomatizations and a Noncooperative Interpretation of the $\alpha$-CIS Value. Asia Pac J Oper Res 32: 5 (2015).

[98] Xu G. Driessen T.S.H. and Sun H.: Matrix analysis for associated consistency in cooperative game theory. Linear Algebra Appl 428, 1571-1586 (2008).

[99] Xu G.,van den Brink R., van der Laan G., and Sun H.: Associated consistency characterization of two linear values for TU games by matrix approach. Linear Algebra Appl 471, 224-240 (2015).

[100] Xu N., Arthur F., and Veinott Jr.: Sequential stochastic core of a cooperative stochastic programming game. Oper Res Lett. 41, 430-435 (2013). 
[101] Yan-An Hwang: Associated consistency and equal allocation of nonseparable costs. Econ Theory 28, 709-719,DOI: 10.1007/s00199-0050640-7 (2006).

[102] Young H.P: Monotonic solutions of cooperative games. Int J Game Theory 14, 65-72 (1985).

[103] Young H.P., Okada N. and Hashimoto T.: Cost allocation in water resources development. Water Resour Res 18, 463-475 (1982). 


\section{Acknowledgements}

The results presented in this thesis have been established in cooperation with several researchers in game theory. Their suggestions and inspiration always encourage me to keep going. I would like to express my appreciation to everyone who has helped me in the last five years.

First of all, I would like to thank Professor Hao Sun, my supervisor in NPU. I got to know Professor Hao Sun when he taught us Probabilistic Theory in 2011. His patience and humour really attracted us. After obtaining my Bachelor degree, I was selected to be a PhD Candidate of Professor Hao Sun. He lead me to the field of game theory and provided me invaluable advice on research. He always supported and encouraged me to attend many academic conferences. He supported me to apply for the scholarship from CSC and visit UT, which extremely broadened my research horizons.

I would like to thank Professor Marc Uetz and dr. Water Kern, my promotor and co-supervisor in UT. Studying abroad is really a challenge, but I feel so lucky to have them as my supervisors. They were always patient to discuss with me. I could always get a quick response from them if I had any problem, which made me feel like home. Thanks to their kindness and constructive suggestion, I could quickly get used to the new environment and successfully complete my $\mathrm{PhD}$ study. I have learned so much from them during the one and half year in Netherlands.

Thanks must go to Professor Dongshuang Hou, a researcher full of knowledge and passion. I couldn't even get my first paper published without his guidance. He taught me the skills how to write a research paper and always 
gave me some important inspirations of research. I really enjoyed the discussions with him. I also appreciate Professor Genjiu Xu from NPU, who always enlightened us during the seminars. He has put much effort in organising conferences in game theory, which benefited many people in this field. I would also like to thank all my colleagues in both NPU and UT. We learned from each other and we made progress together, and they indeed gave me lots of good memories.

Most importantly, I would like to express my sincerely appreciation to my parents, my sisters, my girlfriend, my cute niece and nephew. Without their encouragement and support, I definitely couldn't make it today. I will be there for all of you in the future!

Panfei Sun

November 2018, Enschede 


\section{About the Author}

Panfei Sun was born on April 21, 1993 in Shenqiu Country, Zhoukou city, P.R. of China. From 1998 to 2003, he studied in an elementary school near his village. In order to let him receive a better education, his parents sent him to a boarding school in 2004 . So he could only go back home every two weeks. He then moved to Zhengzhou, the capital of the province, to live with his parents and finished his last year of elementary education. From 2005 to 2010, he completed his middle and high school period in Zhengzhou No.41 school.

In 2010, he was accepted by Northwestern Polytechnical University as a Bachelor student in Applied Mathematics. In 2014, after obtaining his Bachelor degree, he became a $\mathrm{PhD}$ candidate under the supervision of Professor Hao Sun. Since September 2017, he began to study in the department of Applied Mathematics in University of Twente. As a joint PhD student in UT, he is under the supervision of Prof. dr. Marc Uetz and dr.Walter Kern. During this period, he was sponsored by the China Scholarship Council. 